\title{
Intention-based Decision Support : A new way of representing and implementing clinical guidelines in a Decision Support System
}

Citation for published version (APA):

Latoszek-Berendsen, A. A. (2013). Intention-based Decision Support : A new way of representing and implementing clinical guidelines in a Decision Support System. [Doctoral Thesis, Maastricht University]. Maastricht University. https://doi.org/10.26481/dis.20130926al

Document status and date:

Published: 01/01/2013

DOI:

10.26481/dis.20130926al

Document Version:

Publisher's PDF, also known as Version of record

Please check the document version of this publication:

- A submitted manuscript is the version of the article upon submission and before peer-review. There can be important differences between the submitted version and the official published version of record.

People interested in the research are advised to contact the author for the final version of the publication, or visit the DOI to the publisher's website.

- The final author version and the galley proof are versions of the publication after peer review.

- The final published version features the final layout of the paper including the volume, issue and page numbers.

Link to publication

\footnotetext{
General rights rights.

- You may freely distribute the URL identifying the publication in the public portal. please follow below link for the End User Agreement:

www.umlib.nl/taverne-license

Take down policy

If you believe that this document breaches copyright please contact us at:

repository@maastrichtuniversity.nl

providing details and we will investigate your claim.
}

Copyright and moral rights for the publications made accessible in the public portal are retained by the authors and/or other copyright owners and it is a condition of accessing publications that users recognise and abide by the legal requirements associated with these

- Users may download and print one copy of any publication from the public portal for the purpose of private study or research.

- You may not further distribute the material or use it for any profit-making activity or commercial gain

If the publication is distributed under the terms of Article 25fa of the Dutch Copyright Act, indicated by the "Taverne" license above, 
Intention-based Decision Support

A new way of representing and implementing clinical guidelines in a Decision Support System. 
INTENTION-BASED DECISION SUPPORT

A NEW WAY OF REPRESENTING AND IMPLEMENTING CLINICAL GUIDELINES IN A DECISION SUPPORT SYSTEM 


\section{Maastricht University}

This dissertation was supported by the School for Public Health and Primary Care (CAPHRI) of the Faculty of Health, Medicine and Life Sciences (FHML) of Maastricht University.

\section{$\mathrm{N} \widehat{\mathscr{w} O}$}

This research has been funded by the Netherlands Organisation for Scientific Research (NWO), in the framework of the Token 2000 project MIA, grant number 634.000.021.

\section{sirs}

SIKS Dissertation Series No. 2013-25

The research reported in this thesis has been carried out under the auspices of SIKS, the Dutch Research School for Information and Knowledge Systems.

\section{[medecs]}

The research reported in this thesis has been carried out in cooperation with Medecs BV, Eindhoven.

ISBN 978-90-9027746-2

Omslagontwerp en boekverzorging: Sjoerd Diepen en Jos Bruystens

Druk: Print Service Ede

Het drukken van dit proefschrift wordt gesponsord door Tiobe BV

(C) 2013 Agnieszka Latoszek-Berendsen 


\title{
Intention-based Decision Support
}

\author{
A new way of representing and implementing \\ clinical guidelines in a Decision Support System
}

PROEFSCHRIFT

Ter verkrijging van de graad van doctor

aan de Universiteit Maastricht

op gezag van de Rector Magnificus

Prof. dr. L.L.G. Soete

volgens het besluit van het College van Decanen,

in het openbaar te verdedigen

op donderdag 26 september 2013 om 14.00 uur

door

Agnieszka Anna Latoszek-Berendsen 


\section{Promotoren}

Prof. dr. ir. A. Hasman (Academisch Medisch Centrum Amsterdam)

Prof. dr. A.P.M. Gorgels

Prof. dr. H.J. van den Herik (Universiteit Leiden)

Beoordelingscommissie

Prof. dr. G.G. van Merode (voorzitter)

Prof. dr. A. Abu-Hanna (Academisch Medisch Centrum Amsterdam)

Prof. dr. H.P. Brunner-La Rocca

Prof. dr. H.H.M. Korsten (Technische Universiteit Eindhoven)

Prof. dr. G.D.E.M. van der Weijden 


\section{Contents}

CHAPTER 1

CHAPTER 2

CHAPTER 3

CHAPTER 4

CHAPTER 5

CHAPTER 6

CHAPTER 7
General introduction

7

From clinical practice guidelines to computer-interpretable

guidelines. A literature overview

Design and Implementation of GASTINE

69

Implementing guidelines in GASTINE

83

GASTINE: Verification of the system and implemented

99

heartfailure guideline

GASTINE: Intention-based DSS in practice; results of a

121

validation test

General discussion and conclusions

141

Summary

157

Samenvatting

163

Streszczenie

169

Curriculum Vitae

177

Publications

181

Acknowledgement

185

SIKS Dissertation Series

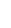

69





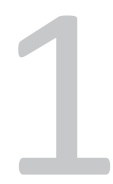

Introduction 
$\frac{8}{\underline{a}}$ 
This thesis focuses on the use of clinical practice guidelines implemented into a computer. Such a computer-based system can support the user and help him in making a variety of decisions. Such a system is called Decision Support System (DSS).

This thesis proposes a solution for building flexible and intuitive guideline-based Decision Support Systems.

\subsection{The world of healthcare}

Healthcare is a domain with many different fields and specializations. Currently, we observe several changes that take place in that domain. Here we mention four important ones: (1) paper patient records are being replaced by electronic patient records (EPR); (2) workflows and treatments are computerised; (3) quality issues have become a major topic: physicians increasingly work in an evidence-based way; (4) the growth of medical knowledge is so fast that most physicians are not able to keep up with this development and have to be supported by knowledge-based systems. With these changes, new challenges and obstacles appear.

One of the greatest challenges of this moment is the way healthcare is struggling with the dilemma of providing the best healthcare (process) of high quality (outcome) at the same or lower costs. Healthcare providers, insurance companies, and patients all have requirements that are often in conflict with each other. For instance, the insurance companies aim at (1) structured work with standardization of treatments and (2) the possibility of saving costs, while healthcare providers and patients emphasise (3) the need for more flexibility in making choices and (4) better patient outcomes [1-3].

One of the disciplines that try to help healthcare to find a balance between those requirements is medical informatics (MI). As active participants in this field, medical informaticians are interested in supporting healthcare with services based on Information and Communication Technologies (ICT).

Medical informatics, called by Greenes et al. in 1990 [4] "the study of computers in medicine" became more than that. It became a discipline at the crossroads of information science, computer science, and healthcare. It deals with resources, medical knowledge, devices, information, workflows, research, and decision support. In this field of healthcare we carried out five studies. The methodology for each study is discussed in the corresponding chapters 2 to 6 .

Further in this chapter, the following topics will be briefly introduced: Decision Support Systems (DSSs), Guidelines and protocols, The problem statement, Intentions, Research questions, and Outline of the thesis. 


\subsection{Decision support systems}

Historical information about the kinds of decision support approaches used in medical informatics and clinical decision making is adequately summarised by Shortliffe [5]. The book by Greenes [6] provides a more recent overview. Decision support initially used decision trees, statistical pattern recognition approaches (Bayesian classification, linear discriminant analysis, etc.) and algorithmic approaches. In medicine, relations between signs and symptoms on the one hand and diseases on the other hand, often cannot be expressed using symbolic logic alone. Because of the remaining uncertainties, statistical approaches were necessary. However, many investigators opined that for solving more complex problems classic probability approaches such as Bayes' rule were not feasible anymore; the approach had to assume conditional independence to be tractable. Yet, the drawback was still that the collection of all the necessary conditional probabilities would be costly and time consuming. Therefore ad-hoc ways of approximate reasoning were devised. The certainty factor approach of MYCIN, the evoking strength/frequency weight model of INTERNIST, or the use of an approximation to Bayes' rule in the PROSPECTOR system, are examples. Shortliffe [5] mentions that in the 1980s interest in the use of probabilistic approaches resurged. Such approaches became more feasible because of improvements in computer hard- and software.

Today, systems that combine formal normative models based on probability and decision theory are used as effective decision support systems. Fox et al. [7], in a commentary to an article by Sittig et al. [8] about 'Grand challenges for decision support', state that empirical approaches to the design of DSSs should be accompanied by sound theoretical principles and safe engineering methods. They point out the importance of the use of decision theory, process theory, knowledge theory, and organisation theory. When discussing the results of the systematic review of the DSS performance by Garg et al. [9], Fox et al. note that the successful Clinical Decision Support Systems (CDSSs) offer functionalities such as providing reminders or alerts, supporting information search, information retrieval and supplying filtering services. They believe that as many elements of the clinical decision process as possible should be supported. Next to the ones mentioned above, also decision making, workflow management, care planning, and monitoring should be available as functions. Guideline modelling languages are envisaged and already developed to provide formalisms for specifying decisions in the context of workflow and care planning. 


\subsection{Guidelines and protocols}

Guidelines are among us for more than 30 years. They provide a specific kind of decision support. Initially the guidelines, or the more algorithmic protocols, were only used by nurses and other ancillary personnel. Many physicians did not use guidelines because of the fact that individual patients differ from each other and guidelines usually cover a population. However, quality issues and patient safety issues have somewhat changed the attitude towards guidelines. Still, guidelines are not used on a large scale by physicians. A variety of barriers to the use of guidelines have been described. Some of the barriers were directly related to the guideline, e.g., the guidelines could contain ambiguous text or even inconsistencies. Other reasons why guidelines were not used frequently were, e.g., (1) paper-based guidelines are difficult to use in clinical practice, (2) physicians consider the use of guidelines as cookbook medicine, (3) physicians are not convinced that the use of guidelines will lead to better care, and (4) organisational barriers [10].

Below we briefly discuss the relevant definitions (subsection 1.3.1), then discuss the computer-interpretable guidelines (subsection 1.3.2) and further introduce the GASTON framework (subsection 1.3.3).

\subsubsection{Definitions}

Guidelines (GL) or Clinical Practice Guidelines (CPGs) are defined as "systematically developed statements to assist physician and patient decisions about appropriate healthcare for specific circumstances" [11].

In addition to guidelines, protocols and clinical pathways were created. The protocol is a detailed written set of instructions to guide the care of a patient or to assist the practitioner in the performance of a procedure [12]. Clinical Pathways are structured, multidisciplinary plans of care designed to support the implementation of clinical guidelines and protocols. They are designed to support clinical management, clinical and non-clinical resource management, clinical audit, and also financial management. They provide detailed guidance for each stage in the management of a patient (e.g., treatments, interventions) with a specific condition over a given time period, and include progress and outcome details [13].

\subsubsection{Computer-interpretable guidelines}

CPGs could be of benefit to physicians by providing an adequate overview of available treatments with recommendations for those who are uncertain about how to proceed. They may too, improve consistency of care and help to change the attitude of practitioners who became used to their, maybe outdated, medical practice.

Early guidelines were often based on consensus among experts from the same field. 
Gradually, this changed to consensus among experts from different fields. However, as mentioned above CPGs could contain ambiguities and inconsistencies. Moreover, consensus does not mean that always the best actions were suggested. Therefore, guideline recommendations were increasingly based on evidence instead of consensus. Research findings were translated into actionable recommendations for clinical care. Since the CPGs were difficult to use in practice the use of computer-interpretable guidelines (CIGs) was considered as alternative. To obtain a CIG, a guideline has to be translated using a formal language and then implemented into a computer model. The creation and use of computer-interpretable guidelines not only needs a formal language, but a whole framework for incorporating the formalised guideline into a decision support system so that it can provide physicians with the relevant information during the process of care. Young et al. [14] present nine requirements for such a framework. These requirements are listed below together with a short explanation as given by Young.

1 Retrieval of guidelines - when several potential guidelines exist, a guideline first must be retrieved from a guideline's repository and perhaps tested for its applicability prior to its application.

2 Visualization of guidelines - to assist the care provider to understand the guideline better, the guideline encoded knowledge should be visualised in an unambiguous and intuitive manner.

3 Integration with local clinical hosts - the GL DSS should be linked with a local clinical information system (e.g., an order entry system), including the user interface, via standard messages and protocols.

4 Persistent storage of guideline applications - to support an intermittent application of complex GLs over long periods of time, the data gathered during application of the GL (e.g., user's decisions) must be stored in a persistent manner (e.g., database).

5 Autonomous clinical control - the DSS must allow the clinician to override or ignore the GL's advice or data interpretation.

6 Support for "look ahead" - to support queries about the meaning of recommended actions, the DSS should enable its users to explore dynamically what the guideline requires in each future situation (i.e., a "What If" query) without actually applying it.

7 Minimise user interaction - due to the short duration of clinical consultations, the DSS must not require too much user attention. For example, the system should indicate when sufficient data have been provided for the current decision step, without requesting an unnecessary data entry by the user.

8 Capability to handle variable access to electronic patient data-the DSS should be able to profit from having an Electronic Patient Record (EPR), in that case it should be able to upgrade its level of support accordingly; but it should also enable sufficient 
application of the GL, even in the situation of not having optimal EPR-based support, or when EPR is not available at all.

9 Capability to handle different levels of guideline representation - the DSS should be able to handle GLs specified at different levels of representation, ranging from structured text to a fully formal, machine-understandable format.

\subsubsection{GASTON framework}

In our research we use the GASTON system; therefore we determined whether the GASTON framework satisfies the above described requirements. GASTON was designed and developed at the Medical Informatics department at Maastricht University and the Signal Processing Systems division of the Department of Electrical Engineering of the Eindhoven University of Technology in the Netherlands. GASTON is a tool for representing and executing computerised clinical guidelines [15]. It consists of three parts: (1) a guideline editor, by which formalised medical guidelines are created; (2) a knowledge base, in which ontologies about medical knowledge are stored; and (3) an execution engine, that during runtime extracts data from the Electronic Patient Record (EPR) and that uses these data to determine, on the basis of guideline information, the action that is the most appropriate for the current patient.

GASTON satisfies eight of the nine requirements listed above. Only requirement 6, "Look ahead" is not implemented. Let us briefly review how those requirements were implemented in GASTON.

1 Retrieval of guidelines - since each guideline contains applicability criteria, they can be used to retrieve the correct guideline when multiple ones on a certain subject are available. In GASTON terms, this means that each guideline/intention has a number of preconditions that determine their applicability.

2 Visualization of guidelines - guidelines are created as a flowchart using components with predefined logic, so called primitives [16]. In GASTON the following types of primitives are defined that are commonly used to describe guidelines: (1) action primitives that specify clinical actions (e.g., administer a drug), (2) decision primitives that model decision points in a guideline (e.g., if this patient suffers from hypertension then perform an action), (3) branching primitives that direct the guideline flow to multiple (parallel) paths and (4) synchronization primitives that converge paths that previously diverged because of a branching primitive [15].

3 Integration with local clinical hosts - the communication between GASTON and patient information systems (e.g., EPRs) takes care of multiple domain ontologies and terminologies, used by the patient information systems. There are a number of standard interface components that are able to communicate with various types of databases and information systems (e.g., ODBC, HL7, and XML). New standards that 
are currently being developed must be incorporated in modern information systems as well as DSSs [17].

4 Persistent storage of guideline applications - user's decisions are stored in a database (e.g., SQL, Oracle), if data is only needed for the length of one session, it is stored in a virtual table in GASTON.

5 Autonomous clinical control - when the user receives an alert message (popup, email, sms) the warning can always be overruled by the clinician. However, it depends on the guideline design whether the same warning is presented multiple times.

6 Support for "look ahead" - the GASTON framework does not support a "look ahead" option, however it supports backtracking. Backtracking systems would show the user the path that the system took to arrive at the current advice.

7 Minimise user interaction - the GASTON system queries other systems (e.g., laboratory, medication) and exploits necessary data to provide optimal advice.

8 Capability to handle variable access to electronic patient data - when data received from other clinical hosts is not sufficient to create optimal advice, GASTON can, in a dialog with the user, ask for missing data. In the situation when the EPR is not available at all, it can give support, e.g., in the form of a dynamic questioner.

9 Capability to handle different levels of guideline representation - Next to representation of computerised guidelines, GASTON enables the use of pictures and hyperlinks, and in this way it supports the user by presenting the relevant content of paper guidelines or other evidence-based materials.

\subsection{Problem statement}

Above we presented the nine requirements of a framework needed to create and use CIGs. The DSSs that emerged were able to provide physicians with information relevant for the current patient. But still, these DSSs are not sufficiently flexible. For instance, when clinical research will lead to new insights or new pharmaceuticals become available, it should be implemented in the system, preferably directly and easily. Therefore, the system should be able to adopt these new insights in a procedural way. Also, the guideline must be implemented in a particular clinical setting. Ultimately, all clinical decisions are made in a local environment, which includes the practice culture, the characteristics of the particular clinician, and the characteristics of the particular patient. It seems paradoxical to produce practice guidelines to reduce variations in practice and then integrate mechanisms that facilitate local variations. The crux is that unwarranted practice variation should be reduced but that often there is need for warranted practice 
variation for very good reasons e.g., multi-morbidity. However, it seems to be the way and it seems to be necessary in order to achieve clinician acceptance [18].

When physicians prescribe medications that are in the spirit of the guideline, current systems will warn the physician that $\mathrm{h}^{1}$ is not working according to the guideline.

In this context an example of the way a hungry cat finds food as described by Winikoff et al. [19] inspired us with ideas how to change our system - GASTON - to make it more flexible. The example by Winikoff is as follows. The hungry cat knows that there is food on the table and formulates a plan to jump on a chair, and then to jump from the chair to the table. The cat leaps on to the chair successfully. At this point, a nearby human moves the chair so that the cat no longer can leap to the table. The cat (being an intelligent agent) realises this, and revises its plans. The new plan is to leap from the chair to a shelf, then walk along the shelf, and finally leap from the shelf down to the table. However, fortune smiles upon the feline: after leaping up to the shelf it finds that food has been left on the shelf. The opportunistic cat abandons its plan to continue to jump to the table and eats upon the shelf instead.

When circumstances change and the original plan cannot be accomplished, the cat goes for an alternative set of actions, but still with the purpose to reach his goal, getting food. He needs to change his primary plan to fulfil his intention. Similarly, a physician may select an alternative treatment than specified in the guideline because of a patient's specific situation or a local or temporary constraint, not taken into account when the guideline was developed.

Based on this example we assume that if the CIG will be provided with the intentions behind the corresponding actions, the DSS will be able, after the entry of an alternative in the EPR that has the same intention as the one of the CIG, to accept such an alternative. Of course, alternative treatments should be known by the system. Therefore, for each intention available alternatives should be incorporated into the DSS. Also, if during runtime the system does not recognize that an alternative treatment used by a physician has the same intention as the one the DSS follows, this alternative treatment should be entered into the system afterwards, after checking the appropriateness of the alternative. The introduction of intentions can also be used to explain to the interested physician why a certain recommendation was given.

The interaction of a physician and a DSS prompted us to the formulation of our problem statement which read as follows.

Problem statement (PS): What features must a DSS (or the CIG) possess to be more flexible and allow actions in the spirit of the guidelines in order to encompass fully satisfyingly the intentions behind the actions? 


\subsection{Intentions}

Intentions are not a new idea. In their work, Miksch et al. [20] introduced intentions in ASBRU. They define intentions as high-level goals that support special tasks such as critiquing and modification. Intentions are patterns of states or actions to be achieved, maintained, or avoided (ACHIEVE, MAINTAIN or AVOID), during or after the execution of the plan (INTERMEDIATE or OVERALL) [21]. ASBRU is a time-oriented plan representation language that represents clinical guidelines as skeletal plans. Skeletal plans are plan schemata at various levels of detail, capturing the essence of the procedure, but leaving room for execution-time flexibility in the achievement of particular goals. The ASBRU syntax is quite elaborate. Current execution engines for ASBRU only use part of the syntax. To our knowledge intentions cannot be processed by these engines.

According to Sips et al. [22] two types of intentions can be recognised, (1) high-level intentions that specify an intention of an action in a specific context, e.g., "determine the possibility of blood group antagonism" and (2) low-level intentions that specify a context independent intention of an action, e.g., "determine blood group" [22]. The authors explain further that the high-level intentions are related to the intentions of medical protocols and low-level intentions concern a physician's actions and actions mentioned in the medical protocols. For each high-level intention in the protocol, there are one or more (sub)protocols that realise this high-level intention, using other high-level intentions and actions. Sips et al. used low-level intentions to characterise both actions recorded in the EPR and actions in the guideline. They could match $70 \%$ of the EPR action sequences with guideline execution sequences. It is important to realise that the same action can have more than one purpose. It is not clear whether Sips et al. assign more than one low-level intention to an action. As will become clear in this thesis we also make use of context independent purposes of actions to check whether alternative actions can be accepted by the system.

\subsection{Research questions}

In order to answer the problem statement, we seek answers to the following five Research Questions (RQ).

\section{RQ1 - What are high quality guidelines and what is the best way to create them?}

To answer this research question we want to obtain, via a literature review, insight into the factors that influence the design and implementation of guidelines that provide personalised advice. We search for studies that enable us to explain the characteristics 
of high-quality guidelines or provide new advanced methods for guideline formalization, computerization, and implementation. We also want to investigate how the guidelines affect processes of care and patient outcome. Moreover, we want to determine the reasons of low guideline adherence.

RQ2 - What should be the architecture of an intention-based decision support system? Based upon the example by Winikoff et al. [19] we investigate how to develop a framework for clinical practice guidelines that not only allows the representation of best practices, but also facilitates reasoning about acceptable alternatives for those best practices.

$R Q 3$ - Do intentions actually provide more flexibility in making medical decisions?

The goal of this research question is to evaluate whether the proposed architecture for a DSS using intentions actually provides the flexibility we envisaged. The DSS should have runtime features, such as plan recognition and backtracking.

RQ4 - Do intentions help to make changes and updates in a DSS easier?

With this research question we investigate whether new alternatives (e.g., therapies, medication) can easily be inserted into the system.

RQ5 - Do intentions help to reduce the complexity of flowcharts and thinking?

Research question 5 has as goal to investigate whether changing the way of thinking during implementation from "what to do?" to "why to do it?" will make (computerised) guidelines more clear.

\subsection{Outline of the thesis}

Chapter 1 contains a description of the research domain, the problem statement, and the five research questions. Chapter $\mathbf{2}$ describes the evolution of guidelines in healthcare and their implementation in daily work and gives an answer on RQ1. Thereafter, in chapter 3, the thesis focuses on RQ2, i.e., the development of a framework for clinical practice guidelines that not only allows the representation of best practices, but also facilitates reasoning about acceptable alternatives for those best practices. Subsequently, in chapter 4, the thesis briefly answers RQ3. It evaluates the design of the framework for computerised intention-based clinical practice guidelines and describes the implementation of the runtime features such as plan recognition and backtracking. Further, in chapter 5, RQ4 and RQ5 are answered. The chapter presents the results and 
conclusions of several tests considering, e.g., reasoning with intentions, giving alerts, and complexity of the flowchart. In chapter 6 results of the validation tests are presented that extensively give the answer to RQ3. Finally, in chapter 7, the findings of our research are summarised, the answers to the problem statement and conclusions are presented, and possibilities for future work discussed. 


\section{References}

1 Woolf SH, Grol R, Hutchinson A, Eccles M, Grimshaw J. Clinical guidelines: potential benefits, limitations, and harms of clinical guidelines. BMJ. 1999 Feb 20;318(7182):527-530.

2 Martens JD, Werkhoven MJ, Severens JL, Winkens RA. Effects of a behaviour independent financial incentive on prescribing behaviour of general practitioners. J Eval Clin Pract. 2007 Jun;13(3):369-373.

3 Cabana MD, Rand CS, Powe NR, Wu AW, Wilson MH, Abboud PA, et al. Why don't physicians follow clinical practice guidelines? A framework for improvement. JAMA. 1999 Oct 20;282(15):1458-1465.

4 Greenes RA, Shortliffe EH. Medical informatics. An emerging academic discipline and institutional priority. JAMA. 1990 Feb 23;263(8):1114-1120.

5 Shortliffe EH. Medical informatics and clinical decision making: the science and the pragmatics. Med Decis Making. 1991 Oct-Dec;11(4 Suppl):S2-14.

6 Greenes RA. Clinical Decision Support. The Road Ahead.: Elsevier Inc.; 2007.

7 Fox J, Glasspool D, Patkar V, Austin M, Black L, South M, et al. Delivering clinical decision support services: there is nothing as practical as a good theory. J Biomed Inform. 2010 Oct;43(5):831-843.

8 Sittig DF, Wright A, Osheroff JA, Middleton B, Teich JM, Ash JS, et al. Grand challenges in clinical decision support. J Biomed Inform. 2008 Apr;41(2):387-392.

9 Garg AX, Adhikari NK, McDonald H, Rosas-Arellano MP, Devereaux PJ, Beyene J, et al. Effects of computerised clinical decision support systems on practitioner performance and patient outcomes: a systematic review. JAMA. 2005 Mar 9;293(10):1223-1238.

10 Latoszek-Berendsen A, Tange H, van den Herik HJ, Hasman A. From clinical practice guidelines to computer-interpretable guidelines. A literature overview. Methods Inf Med. 2010;49(6):550-570.

11 Field M, Lohr K, (Eds). Guidelines for clinical practice: from development to use. Institute of Medicine, Washington, DC: National Academy Press. 1992.

12 Miller-Keane. Encyclopedia \& Dictionary of Medicine, Nursing \& Allied Health. Seventh Edition ed: Sounders, an imprint of Elsevier; 2003.

13 Campbell H, Hotchkiss R, Bradshaw N, Porteous M. Integrated care pathways. BMJ. 1998 Jan 10;316(7125):133-137.

14 Young $\mathrm{O}$, Shahar Y, Liel Y, Lunenfeld E, Bar G, Shalom E, et al. Runtime application of HybridAsbru clinical guidelines. J Biomed Inform. 2007 Oct;40(5):507-526.

15 de Clercq PA, Hasman A, Blom JA, Korsten HH. Design and implementation of a framework to support the development of clinical guidelines. Int J Med Inform. 2001 Dec;64(2-3):285-318.

16 Ohno-Machado L, Gennari JH, Murphy SN, Jain NL, Tu SW, Oliver DE, et al. The guideline interchange format: a model for representing guidelines. J Am Med Inform Assoc. 1998 JulAug;5(4):357-372. 
17 De Clercq P. Guideline-based Decision Support in Medicine. Stud Health Technol Inform. 2003;146:516-520.

18 Zielstorff R. Online practice guidelines: issues, obstacles, and future prospects. J Am Med Inform Assoc. 1998;5(3):227-236.

19 Winikoff M, Padgham L, Harland J, Thangarajah J. Declarative and Procedural Goals in Intelligent Agent Systems. 2002 Toulouse, France, April 22-25, 2002.

20 Miksch S, Shahar Y, Johnson P. Asbru: A task-specific, intention-based, and time-oriented language for representing skeletal plans. Proceedings of the Seventh Workshop on Knowledge Engineering Methods and Languages. 1997((Milton Keynes, UK, 1997)):9-1 - 9-20.

21 Marcos M, Berger G, van Harmelen F, ten Teije A, Roomans H, Miksch S. Using critiquing for improving medical protocols: harder than it seems. In Proc of the 8th European Conference on Artificial Intelligence in Medicine (AIME-2001). 2001:431-441.

22 Sips RJ, Braun L, Roos N. Enabling protocol-based medical critiquing. Stud Health Technol Inform. 2006;124:471-476. 


\section{From clinical practice guidelines to computer-interpretable guidelines. A literature overview}

Published as:

Latoszek-Berendsen AA, Tange H, van den Herik J, and Hasman A. From clinical practice guidelines to computer-interpretable guidelines. A literature overview. Methods Inf 


\section{Abstract}

\section{Background}

Guidelines are among us for over 30 years. Initially they were used as algorithmic protocols by nurses and other ancillary personnel. Many physicians regarded the use of guidelines as cookbook medicine. However, quality and patient safety issues have changed the attitude towards guidelines. Implementing formalised guidelines in a decision support system with an interface to an Electronic Patient Record (EPR) makes the application of guidelines more personal and therefore acceptable at the moment of care.

\section{Objective}

To obtain, via a literature review, an insight into factors that influence the design and implementation of guidelines.

\section{Methods}

An extensive search of the scientific literature in PubMed was carried out with a focus on guideline characteristics, guideline development and implementation, and guideline dissemination.

\section{Results}

We present studies that enable us to explain the characteristics of high quality guidelines, and new advanced methods for guideline formalization, computerization, and implementation. We show how the guidelines affect processes of care and the patient outcome. We discuss the reasons of low guideline adherence as presented in the literature and comment upon them.

\section{Conclusions}

Developing high quality guidelines requires a skilled team of people and sufficient budget. The guidelines should give personalised advice. Computer Interpretable Guidelines (CIGs) that have access to the patient's EPR are able to give personal advice. Because of the costs, sharing of $\mathrm{CIGs}$ is a critical requirement for guideline development, dissemination, and implementation. Until now this is hardly possible, because of the many models in use. However, some solutions have been proposed. For instance, a standardized terminology should be imposed so that the terms in guidelines can be matched with terms in an EPR. Also, a dissemination model for easy updating of guidelines should be established. The recommendations should be based on consensus on evidence instead of consensus on opinions only. To test the quality of the guideline, appraisal instruments should be used to assess the guideline as a whole, as well as checking the quality of the recommendations individually. Only in this way optimal guideline advice can be given on an individual basis at a reasonable cost. 


\subsection{Introduction}

Clinical practice guidelines (CPGs) are meant to improve the process and outcome of healthcare and to optimise resource utilization. CPGs are defined as systematically developed statements to assist physician and patient decisions about appropriate healthcare for specific circumstances [1].

The development of CPGs was a response to the discovery of large, unexplained variations in (1) the practice of the physician, (2) documentation of significant rates of inappropriate care, and (3) an interest in managing healthcare cost [2]. Important is the word assist: guidelines are guides rather than rules. In contrast, clinical protocols provide a comprehensive set of rigid criteria outlining the management steps for a single clinical condition or other aspects of the organisation [3]. The text of CPGs usually contains a number of components such as: the intention(s) of the guideline, the medical background, patient eligibility criteria, procedural statements (e.g., drug recommendations), evidence for the advisories, treatment cost-benefit analyses, and references [4].

Guidelines are already among us for more than 30 years. Initially, the guidelines or the more algorithmic protocols were only used by nurses and other ancillary personnel. Many physicians did not use guidelines because individual patients differ from each other and guidelines usually cover a population. However, quality issues and patient safety issues somewhat changed their attitude towards guidelines. But, still, physicians do not use guidelines on a large scale. A variety of obstacles to the use of guidelines have been discovered. Some of the obstacles were directly related to the guideline: for example, the guidelines could contain ambiguous text or even inconsistencies. Other reasons were, e.g., (1) paper-based guidelines are difficult to use in clinical practice, (2) physicians consider the use of guidelines as cookbook medicine, (3) physicians are not convinced that the use of guidelines would lead to better care, or (4) organisational barriers are present.

With the advent of decision support systems, including guideline implementation systems, the situation changed drastically. Formalised and computer interpretable guidelines (CIGs) could be made (more) patient specific. By coupling a guideline implementation system to an Electronic Patient Record (EPR), alarms and reminders could be issued concerning the management of an individual patient. Computer-based guidelines can, to a certain extent, be automatically checked to discover inconsistencies or open ends. In this way the quality of the guidelines can be raised. Also, the compliance to guidelines can be checked by formal methods.

Early guidelines were often based on consensus among experts. Later guideline recommendations were increasingly more evidence-based. So, research findings were translated into actionable recommendations for clinical care. Since the scientific quality of 
the sources of the evidence on which the recommendations are based can vary, evidence is classified into a number of levels. For the purpose of guideline development usually three levels are distinguished: A, B, and C. The American College of Cardiology (ACC) and the American Heart Association ( $\mathrm{AHA}$ ) taskforce on practice guidelines also recognize the three levels of evidence [5].

Level A concerns data derived from multiple randomised clinical trials, level B concerns data derived from a single randomised trial or non-randomised studies, and level $\mathrm{C}$ concerns a consensus opinion of experts.

The taskforce emphasises the importance of (1) mentioning the levels of evidence of the sources on which recommendations are based, and of (2) classifying the confidence in the recommendations itself. Three classes of recommendations are distinguished. Class I contains conditions for which there is evidence and/or general agreement that a given procedure or treatment (according to the recommendation) is useful and effective, Class II contains conditions for which there is conflicting evidence and/or a divergence of opinion about the usefulness/efficacy of a procedure or treatment, whereas Class III contains conditions for which there is evidence and/or general agreement that the procedure is not useful/effective, and in some cases may be harmful. By stating the level of evidence of the sources used for the recommendations, and the classes of recommendations, users of guidelines become aware of the strength of the research evidence supporting each recommendation mentioned in the guideline.

Below we provide three different groups of caveats (2.1.1) and an overview of the paper (2.1.2).

\subsubsection{Caveats regarding the use of CPGs}

CPGs could be of benefit for physicians because they receive a better overview of available treatments with recommendations for those who are uncertain about how to proceed. Obviously, guidelines may improve consistency of care and help to change the attitude of practitioners who became used to their, maybe outdated, medical practice. However, in situations where CPGs are flawed or misleading, professionals can be harmed by them. In the following, we list three different groups of concerns, expressed by the authors mentioned below, with respect to guidelines.

First, the type of advice in guidelines is influenced by the experience and knowledge of the guideline developing group, since only a small subset of what is recommended in guidelines usually has been tested in well designed studies. The proportion of recommendations for which there is no conclusive evidence is also growing. Tricoci et al. [6] determined that recommendations issued in current ACC/AHA CPGs are largely developed from lower levels of evidence or expert opinion. The authors highlighted the need to improve the process of writing guidelines and to expand the evidence base 
from which CPGs are derived. Here we remark that Tricoci et al. were aware that their findings are reflective of a specialty that has a large pool of research to draw on for its care recommendations.

Second, guidelines in medical areas, in which large clinical trials are performed less frequently, may have an even weaker evidence-based foundation. Therefore, there is a risk for professionals that auditors or managers may unfairly judge the quality of their care based on criteria from invalid guidelines. Shaneyfelt and Centor placed this problem in the spotlight in an editorial in JAMA [7]. The authors were concerned that some of the consensus statements taken out of the guidelines will be turned into performance measures and other tools to critique the quality of physician care.

Third, Shaneyfelt and Centor [7] noted that guidelines often have become marketing tools for device and pharmaceutical manufacturers and that financial ties between guideline panel members and industry are common.

\subsubsection{Goal and overview}

In this chapter we present a literature survey of factors that influence the design and implementation of guidelines. In section 2.1, we described our motivation for this literature search (improving quality of care and patient safety taking into account the three groups of serious caveats mentioned above). In section 2.2, we present the method of literature search. Since only high quality guidelines will probably be used in practice, in section 2.3, we discuss how the quality of guideline development and implementation can be assessed. Then in section 2.4, we describe formalization methods to translate paper guidelines into computer-based guidelines. Further on in section 2.5, we introduce models for computer interpretable guidelines that facilitate their design. Additionally, we discuss problems that may occur during the formalization process. Further in section 2.6, we describe the effects of guidelines with respect to patient outcome and efficiency of care (including reasons why guidelines are not used). In section 2.7, we present barriers to guidelines adherence. Finally, we discuss the current state of the art and present our ideas for further challenges in section 2.8 .

\subsection{Methods}

As stated in section 2.1 we concentrate our search of the scientific literature on (1) guideline characteristics, (2) guideline development and implementation, and (3) guideline dissemination. After the following five steps we arrived at our defined set of articles. 
First, an extensive search of the scientific literature in PubMed was carried out in October 2009 using the following key terms: clinical practice guidelines, paper(-) based guidelines, computer(-)based guidelines, computerised (clinical) guidelines, decision support system, computerised guidelines, computer interpretable guidelines, guideline formalization. By using these keywords incorporated in adequately formulated search queries 1727 articles were retrieved. We only took into account articles available with full text. Our query was as follows: (\#1 AND \#4) OR \#2 OR (\#3 AND \#5) OR \#6 where

\#1 Clinical practice guidelines [All Fields];

\#2 Paper-based guidelines [All Fields];

\#3 Computer-based guidelines [All Fields];

\#4 Computerised guidelines [All Fields];

\#5 Decision support system [All Fields];

\#6 Computer interpretable guidelines [All Fields].

Second, the titles of the articles were scanned and those articles were selected that matched at least one of the following five categories.

1 Characteristics of clinical practice guidelines

2 Paper-based guidelines

3 Development: Guideline formalization

4 Implementation: Computer interpretable guidelines, feedback systems, decision support systems

5 Dissemination: approaches facilitating the design of computer interpretable guidelines

Papers in the category 1 should provide information about attributes of clinical practice guidelines, or the definition of a "good" guideline, or mention standards to build clinical guidelines. Category 2 holds articles that contain details about papers-based clinical practice guidelines that focus on guideline acceptance, performance of the guidelines with respect to patients' outcome and with respect to the process of care. Category 3 contains articles that describe how to translate paper-based guidelines to computerbased guidelines. Category 4 captures papers that describe feedback systems and decision support systems, and/or includes effects of those systems. Category 5 includes papers that present methods for developing computer-based guidelines as well as decision support systems that incorporate these guidelines.

In total 696 articles matched the categories.

Third, the abstracts of those papers were independently studied by two evaluators (author 1 and 4 of this article, both being medical informaticians). This resulted in a set of 276 articles.

Fourth, the content of those 276 articles was examined. After reading the full papers, this set was reduced to 83 papers. 
Fifth, by screening the references in these articles and reading new available sources published in 2010, 20 papers were added to the set that fulfilled the criteria mentioned above.

\subsection{Quality of guidelines}

Research suggests that physicians do not extensively use CPGs at the point of care, because they are quite time consuming to go through and unsuitable for just-in-time use. To overcome this obstacle many guidelines are therefore accompanied by algorithms which represent practice recommendations in diagrammatic form. Since the recommendations in paper-based guidelines do not always reflect the flow of patient encounters, the diagrams are portrayed as flowcharts to specify the recommended steps. With this addition recommendations can be extracted quickly. A disadvantage is that because of the condensed nature of the algorithms they are often rigid and cannot provide all the information present in the text-based guidelines which may be needed by non-expert physicians. Also, there is no room for explanation of counter-intuitive advice [8].

Guidelines based on the synthesis of the best, most recent evidence can help practitioners keep current with the literature and help them assimilate evidence into practice [9]. Given the resources required to identify all relevant primary studies many guidelines rely on systematic reviews that were either previously published or carried out by the guideline developers themselves. Systematic reviews can aid in guideline development because they involve searching for, selecting, critically appraising, and summarising the results of primary research. However, sole reliance on systematic reviews will never adequately serve the development of guidelines. Guideline developers still have to appraise the original studies to understand the populations, interventions, and outcomes evaluated. When several systematic reviews exist on the same topic they may generate conflicting conclusions and then the developers have to deal with such information in a transparent way.

Yet, many guidelines represent expert consensus [7]. Since guideline committees begin with implicit biases and values, the recommendations they make may be affected. Bias may occur subconsciously and go unrecognised. Guideline users can only adjust for biases when guideline panels make their values and goals explicit, but usually they remain opaque.

Below we discuss characteristics of high quality guidelines (2.3.1), guideline appraisal instruments (2.3.2), aspects of guidelines maintenance (2.3.3), and guideline dissemination and implementation (2.3.4). 


\subsubsection{Characteristics of high quality guidelines}

Evidence-based guidelines can reduce the delivery of inappropriate care. Several medical organisations have published methodological standards for developing scientifically sound guidelines. Below we review four of such standards and opinions on what good quality may constitute. We emphasise the standards for computer interpretable guidelines.

(1) The American Institute of Medicine (IOM) defined eight desirable attributes of CPGs: validity, reliability and reproducibility, clinical applicability, clinical flexibility, clarity, documentation, development by a multi-disciplinary process, and plans for review [1] However, critical information that would attest to validity or would document fulfilment of the other IOM criteria is regularly absent from published guidelines [2]. For example, ambiguity and vagueness in the wording of the recommendations, incomplete decision logic pathways, and poor differentiation between evidence and opinion are common shortcomings of many guidelines [10].

(2) Littlejohns and Cluzeau [11] define a 'good' guideline as a guideline that is valid, reproducible, and reliable. This means that there is evidence that (i) a guideline, when implemented, will lead to outcomes that it intended to achieve; (ii) given the same data, other guideline producers will offer the same recommendations, and (iii) guideline users will interpret the recommendations in the same way.

(3) Burgers et al. [12] define the quality of a guideline as the confidence that the potential biases inherent of guideline development have been addressed adequately and that the recommendations are both internally and externally valid, and are feasible for practice.

(4) Shiffman et al. [13] observe that the quality of guidelines is related primarily to the scientific validity of guidelines and that, generally, the quality is assessed for the guideline as a whole. According to the authors the implementability of guidelines also should be assessed. Implementability is the aspect of guideline quality that indicates how easily individual recommendations within a guideline can be implemented in practice. Two dimensions of implementability are particularly important: executability (do the recommendations communicate what to do) and decidability (do the recommendations state when to do it). In addition, the aspect computability indicates the ease by which a recommendation might be operationalised in an electronic information system.

\subsubsection{Guideline appraisal instruments}

The process of guideline creation is time- and resource- consuming. To be sure that the guidelines will be used in clinical practice, they have to be of the highest quality. Therefore, appraisal instruments have been developed to guide the development process and to evaluate the quality of the developed guidelines.

Below, we present four guideline appraisal instruments that help to assess the quality of guidelines. 
(1) To assess the methodological quality of guidelines published in the peer-reviewed medical literature, Shaneyfelt et al. [2] developed and validated a 25-item guideline appraisal instrument. The instrument assessed the guidelines with respect to standards on format and development (10 items), identification and summary of evidence (10 items), and formulation of recommendations (5 items). They concluded that on average the 279 evaluated guidelines published in the peer-reviewed medical literature from 1985 to 1997 adhered to $43.1 \%$ of the 25 items. Guidelines show significant improvement over the years (from $36.9 \%$ in 1985 to (still only) 50.4\% in 1997). Although guidelines are developed to improve health outcomes only $40 \%$ of the guidelines specified the outcomes of interest. Also, less than half of the guidelines described the population to which the guideline should be applied. The standards on the identification and summary of evidence were poorly followed (only one third of the guidelines adhered to these standards). Just few of the guidelines (14.3\%) quantified the effects on healthcare costs. Most guidelines (82.1\%) specified the preventive, diagnostic, or therapeutic options available.

(2) The AGREE (Appraisal of Guidelines for Research and Evaluation) instrument [14] was developed to assess both the quality of the reporting and the quality of some aspects of the recommendations of the guideline. It provides an assessment of the validity of a guideline. In total six predictors (scope and purpose, stakeholder involvement, rigor of development, clarity and presentation, applicability, and editorial independence) are considered by the instrument. A four-point Likert scale is used to score each of the 23 items.

Burgers et al. [12] sought to identify which of these predictors have the most impact on guideline quality. The quality of a guideline was determined by the AGREE instrument and scores were obtained for the six predictors that are considered in the instrument. The data collected for the validation of the AGREE instrument was analyzed. In total 86 guidelines developed by 62 different agencies from 11 countries were assessed using the instrument. Burgers et al. concluded that the type of developing agency was a major predictor. Guidelines produced within established guideline programs (e.g., structured and coordinated programs designed with the specific aim of producing several clinical practice guidelines) and by government-funded agencies had a higher quality than the guidelines produced by specialist societies. This is due to the fact that governmentfunded agencies have a guideline program that provides a systematic procedure with key elements such as a multidisciplinary guideline development group, systematic literature review, external peer review, and different products for dissemination. The influence of the other five investigated predictors of guideline quality was limited. The study also showed that although the type of developing agency was a good predictor, the agency scored lower on the AGREE quality domain "applicability" (applicability pertains to the likely organisational, behavioural and cost implications of applying the guideline) than 
on the other quality domains. Apparently agency policies and procedures are more concerned with the methodology of producing guidelines than with the effectiveness of guidelines in daily practice.

(3) Shiffman et al. [15] convened the Conference on Guideline Standardization (COGS) to define a standard (the COGS statement) for guideline reporting that would promote guideline quality and facilitate implementation. In a modified Delphi study, participants had to rate their agreement with the statement that "[Item name] is a necessary component of practice guidelines". This study resulted in the COGS statement, a checklist of 18 topics. Specifically one of the purposes was to address problems with implementing guidelines electronically. Guideline authors strive to make recommendations that accurately reflect the scientific evidence but sometimes they intentionally introduce ambiguity into the recommendations to reflect their uncertainties. Often, developers use terms that are not clearly defined, thereby presenting difficulties when recommendations are integrated into a decision support system. The use of the checklist for a productive discussion among (1) guideline developers, (2) disseminators and implementers, and (3) knowledge managers about critical guideline items and clear statements of decidable and executable recommendations may overcome major impediments to guideline use.

Guidelines that have been reported according to the COGS statement are likely to be easier to assess for validity because they systematically report precise details that are critical for understanding a guideline's development, its recommendation statements, and potential issues in its application [16]. Shiffman et al. [15] note that the COGS checklist represents a common framework for guideline developers to help ensure that important information is included in the guideline documentation. For journal editors and other disseminators, the COGS checklist helps to determine that a guideline draft is ready for publication. For implementers, the checklist is to inform the selection process and to facilitate the representation and translation of recommendations into tools that influence clinical behaviour.

(4) Shiffman et al. [13] developed and validated a tool for appraisal of the implementability of clinical guidelines: GLIA (Guideline Implementability Appraisal). GLIA is intended to help anticipate barriers to implementation success. It can be used by the authors of the guideline and by those individuals who choose guidelines for applications within a healthcare delivery system. As a guideline is being developed GLIA can provide feedback to guideline authors about potentially remediable defects. Implementers can use GLIA to help select a guideline, to identify potential obstacles, and to target efforts toward addressing identified barriers.

Factors that indicate guideline quality but not implementability were not included in GLIA. Implementability was defined to refer to a set of characteristics that predict the relative ease of implementation of guideline recommendations. GLIA focuses on 
factors that are fundamental to the guideline. Extrinsic items relating to the effect of the recommendations on the process of care and items relating to the novelty or innovation of a guideline statement were retained because developers can anticipate these barriers and offer potential strategies for implementation success. The factors were grouped into categories and then specific questions were devised to characterise each of these categories. These questions became items of the instrument. GLIA consists of 31 items, arranged into 10 dimensions. Questions from 9 of the 10 dimensions are applied individually to each recommendation of the guideline. Decidability and executability are critical dimensions [13]. Application of GLIA to the measurement of decidability and executability requires that users translate guideline recommendations into statements comprising conditions and actions. Other dimensions of GLIA are: global (general characteristics of the guideline as a whole), presentation and formatting, measurable outcomes, apparent validity, and flexibility (degree to which a recommendation permits interpretation and allows for alternatives in its execution), effect on process of care, novelty/innovation, and computability.

Several GLIA items overlap with items in the AGREE instrument. However, the GLIA authors claim that GLIA is the only tool that emphasises implementation concerns at the level of the individual recommendation. GLIA incorporates an optional category - computability to indicate the ease with which a recommendation might be operationalised in electronic information systems.

From this overview we may conclude that there are sufficient instruments to evaluate the quality of existing guidelines. These instruments mainly evaluate the quality of the whole guideline. As we will show later, there is a trend now to evaluate the separate recommendations of the guideline rather than the guideline as whole. Some of the instruments not only assess the quality of the guideline as a whole but also (1) the separate recommendations, (2) whether guidelines are adequate for implementation in practice, and (3) whether guidelines can be used by decision support systems.

\subsubsection{Maintenance of guidelines}

In this section we discuss three aspects of maintaining clinical practice guidelines, viz. maintenance, local adaptations, and augmented dissemination model.

Maintenance of guidelines is a notable problem to take into account, since guidelines have to be periodically updated. Tricoci et al. [6] noted with respect to the cardiovascular guidelines of the ACC/AHA that the mean time elapsing from a version to its update was 4.6 years for disease-based guidelines, 5.4 years for interventional procedure-based, and 8.2 years for diagnostic procedure-based guidelines. They also indicate that updated versions are not always more evidence-based than their predecessors. Tricoci et al. concluded that among ACC/AHA clinical practice guidelines with at least one revision or 
update, the number of recommendations increased with $48 \%$, with the largest increase observed in the use of class-II recommendations. In the 16 current guidelines reporting levels of evidence, only 314 of the 2711 recommendations are classified as level of evidence $A$, whereas 1246 are level of evidence $C$. The level of evidence significantly varies across categories of guidelines (disease, intervention, or diagnostic) and across individual guidelines. Recommendations with level of evidence A are mostly concentrated in class I, but only 245 of 1305 class-I recommendations have level of evidence $A$.

Moreover, local adaptations to guidelines make maintenance more difficult. When revised guidelines are disseminated they have to be reintegrated into the local clinical settings. The revisions must be synchronised with the changes previously made to this guideline or a related guideline. Current guideline representations do not provide a sufficiently fine-grained representation for complete identification of revisions in a guideline. This problem again can be attacked better with the help of CIGs.

The Guideline Elements Model (GEM) (see section 4.3) defines elements for identifying whether a guideline has been revised and adapted, but not how. Boxwala et al. [17] suggested enhancing the GEM by adding components that (1) identify the specific elements that have been revised, (2) state the reason for the change, and (3) explain the revision. They proposed an augmented dissemination model. In this model the guideline is downloaded, its content is adapted to local practice preferences and then it is integrated with an institutional clinical information system. When revisions of the guideline are published, the revised guideline is downloaded, unmodified sections of the revised guideline are automatically synchronised with the previous local version of the guideline, and the modified sections are then manually adapted and integrated.

In summary, the update procedure is still in a state of flux, and needs to be improved.

\subsubsection{Guideline dissemination and implementation}

The use of guidelines is determined by (1) the quality of the guideline development (discussed above), (2) by the way the guidelines are disseminated and ( 3 ) the way they are implemented. Points 2 and 3 are discussed below.

For busy clinicians, clinical practice guidelines can only be of help when they take notice of these guidelines. Insight into the best ways to spread the guidelines (dissemination) is therefore important. There are several dissemination and implementation strategies: educational strategies, educational outreach, audit/feedback/peer review, reminder and decision support systems, financial incentives, use of a local opinion leader, and combinations of these approaches (so-called multifaceted interventions). Beneath, we discuss the most prevailing ones.

The effectiveness of guideline dissemination and implementation strategies has been studied in systematic reviews. Below we discuss three important reviews. First, Grimshaw 
et al. [18] concluded that current guideline dissemination and implementation strategies can lead to improvements in care. However, there was considerable variation in the observed effects. Second, in their study Prior et al. [19] came to similar conclusions. Effective strategies must include multifaceted interventions, interactive education, and clinical reminder systems. Didactic education and passive dissemination strategies were ineffective. They concluded that successful strategies should actively engage clinicians throughout this process. Third, a study carried out among general practitioners in the Netherlands, to gain insight into processes of dissemination of guidelines, showed that the scientific journal was the most important, but certainly not the only source of information to become informed about them. Discussing the guidelines in the local family doctor group was also a means of dissemination. It was concluded that segmentation of the target group is necessary for effective dissemination of guidelines: for some doctors it is desirable to make evidence quickly available, for others spreading the guidelines through the local network may be effective, while for a third group a more active, personal approach may be necessary [20].

Below we focus on implementation details.

Guideline implementation involves the concrete activities and interventions undertaken to turn policies into desired results. Guidelines are not uniformly successful in improving care and several instances of implementation failure have been described [13]. Moreover, performance measurement and payment are increasingly linked to goals established by practice guidelines. Lin and Slawson [21] wrote an article on how to identify and use good practice guidelines. We conclude with them that the best guidelines are those based on systematic reviews and patient-oriented evidence, and are prospectively validated. The guidelines should have a transparent development process, identify potential conflicts of interest, and offer flexibility in various clinical situations.

Most CPGs are now easily accessible on specialised websites. For instance, the National Guideline Clearinghouse ${ }^{\mathrm{TM}}$ (NGC) is a public resource for evidence-based clinical practice guidelines. However, the wide diffusion of CPGs does not solve the problem of their effective use in daily practice (implementation).

Returning for a while to dissemination we may remark that disseminating CPGs in a textual format proved to be inefficient [22]. The practitioner has to read several pages in order to find the appropriate care recommendation for a specific clinical circumstance. Their practical aspect rather than their content is then at fault. Guidelines will be much more efficient if they are available in the healthcare setting, integrated in the healthcare information system, easily adaptable to given clinical situations/scenarios and able to avoid overloading physicians with non-essential information. Minimizing the time spent consulting CPGs is crucial when attempting to improve their usage in everyday practice. Computer interpretable guidelines will therefore become a necessity. 


\subsection{Formalization of guidelines}

The effect of clinical practice guidelines (CPGs) and computer interpretable guidelines (CIGs) depends on the proper implementation of either type of guideline. It is unrealistic to expect that clinicians read and use all guidelines that are available; however they are still expected to possess all the knowledge. The increasing number of CPGs and the encountered problems with CPGs was the reason that for over a decade researchers have looked for automated methods of delivering guidelines to clinicians when the guideline is most relevant to the care of patients: when the patient is seen by the clinician. Below we discuss: standardization of decisions (2.4.1), knowledge - based approach (2.4.2), methodological issues (2.4.3), document-centric approach (2.4.4), the bridging approach (2.4.5), and implementation in Decision Support Systems (2.4.6).

\subsubsection{Standardization of decisions}

Morris [23], a proponent of the use of computer-based protocols, declared that standardization of clinical decisions is needed for clinical practice. Without explicitly defining the recommendations and the context in which they are appropriate, the fundamental scientific requirement of replicability of results cannot be achieved. A guideline should not require judgments by a clinician. Any form of guideline or protocol can theoretically contain sufficient detail. In practice, however, paper-based versions of any but the simplest protocols cannot be made explicit and therefore remain dependent on clinician judgment. The need to standardize decisions provides a contrast to the equally compelling need to deliver individualised, patient-specific treatment. Computerbased protocols, which are explicit, detailed, and patient data-driven, can simultaneously achieve standardization of clinical decision making and individualization of patient care.

\subsubsection{Knowledge - based approach}

The translation of paper-based into computer-based guidelines can be done in at least two different ways: (1) in a knowledge-based approach an expert extracts information from the guideline text, interprets it, and then encodes it using one of the guideline models described in section 2.5; (2) in the document-centric approach mark-up methodologies are used to provide guideline text excerpts relevant to the patient context.

The development of clinical guidelines is quite expensive; they should be shared among clinical institutions. Patel et al. [24] described the process of shared guideline development. First, a paper-based guideline has to be created at authoring institutions. Second, paperbased guidelines have to be translated into a computer-based guideline representation language (such as GLIF); GLIF is a computer-interpretable language for modelling and executing clinical practice guidelines. GLIF has a formal representation. It defines ontology 
for representing guidelines, as well as a medical ontology for representing medical data and concepts. GLIF was developed by the InterMed Collaboratory (Stanford Medical Informatics, Harvard University, McGill University, and Columbia University)). Third, the representation has to be implemented within the clinical institution's application system. Fourth, the clinician has to interpret the guideline as it is represented in the guideline applications.

Following the steps of Patel, more groups have translated paper-based guidelines into a computer-based system. Gillois et al. [25] successfully implemented two cardiovascular CPGs and a hypercholesterolemia guideline into a computer system. First, they chose the national evidence-based guideline. Second, they translated it into a linear algorithm. Third, they represented it in GLIF, and fourth, they connected it with an EPR. The system outcome was the advice whether to start treatment or not. Gillois et al. concluded that to improve guideline computability and efficiency it is necessary to use a framework for guideline development.

Guideline developers tend to focus on specific tasks rather than on processes such as care plans and pathways which are extended in time. In contrast, research on business process modelling has demonstrated notions and tools which deal directly with process modelling, but has not been concerned with problems like data interpretation and decision making. Fox et al. [26] described these two approaches (guideline development vs. business process modelling) and compared some of their strengths and weaknesses.

\subsubsection{Methodological issues}

Below, we show the impact of users (2.4.3.1), expert involvement (2.4.3.2), parallel guideline development (2.4.3.3), and used formalisms on guideline development (2.4.3.4).

\subsubsection{User involvement}

Trivedi et al. [27] pointed out that user satisfaction leads to increased use of the system. Increased use of the system leads to end-users that are more efficient. The knowledge of end-users influences their perception of usefulness and it helps to give physicians as much information as possible before implementation. If end-users are left out of the decision making process they will have no personal investment in the use of the new system. User involvement in implementation can improve reliance and utilization.

\subsubsection{Experts involvement}

CPGs need to be developed by experts from different fields. Already Patel et al. [24] in 1998 concluded that guidelines developed by teams that include both clinicians and experts in computer-based representations are preferable to those developed by individuals of either type working alone. At the same time, Woolf [28] (1999) warns that 
guidelines created only by medical or other groups not concerned with financial aspects, may promote costly interventions that are unaffordable or that cut into resources needed for more effective services. There has to be a well defined balance between these matters while developing clinical guidelines. Still, in 2006, Biondich et al. [29] observed that even though clinical guideline authors, health information technology (HIT) developers, standards development organisations, and information system implementers all work to improve the processes of healthcare, they have long functioned independently towards realizing these goals. The independence has led to clinical standards of care that often poorly align with the functional and technical HIT standards developed to realise them. Biondich et al. describe the shortcomings and inefficiencies inherent in the current process and proposed two solutions. The first one is the creation of a technical expert panel. Among this group are representatives from medical informatics, quality improvement experts, disease content experts, and health service researchers. The second solution is the creation of a dictionary that clearly defines all decision variables with written definitions.

\subsubsection{Parallel guideline development}

Patel et al. [24] studied collaboration problems that arise when translating a clinical guideline from text into an encoded form. Guidelines should neither contain too much nor too little information. If they do, it may lead to erroneous and even dangerous interpretations. The translation has to take these considerations into account. Different experts with different backgrounds and expertise may construct different mental models of the guideline. Collaboration may help to develop a shared mental model. However, building a shared model through collaboration may bring conflicts as every individual brings different knowledge and experience to the task. Yet, the negotiation of conflict may be beneficial as experts contribute relevant knowledge as they explain and justify their individual interpretations.

Based on the guideline development principles by Shekelle et al. [30], Goud et al. [31] designed a parallel guideline development strategy, in which a multidisciplinary group of experienced and knowledgeable professionals cooperate in creating both a textual and a computer interpretable guideline. This strategy of parallel guideline development and formalization was applied during the development of the Dutch Cardiac Rehabilitation Guidelines of 2004 and appeared to be successful. In this approach guideline authors and a guideline formalization team closely collaborated. The guideline developers focused on relevant scientific evidence while the guideline formalization team looked for the right approach and tools, needed to formalise the guideline. Parallel guideline development gives an opportunity to eliminate, in an early stage, vague and incomplete concepts and recommendations. 
From the above cited publications we may conclude as Peleg et al. [32] also did that the collaboration between guideline authors and (medical) informatics researchers in the process of developing guidelines, improves the logical consistency and completeness of clinical algorithms or flowcharts. Here we remark that each collaborator brings in its own expertise. Any problems encountered or errors identified during guideline formalization should be directly discussed and, if needed, resolved.

\subsubsection{Guideline formalisms}

Existing guideline formalisms try to create computer interpretable models of clinical guidelines without the need to encode the abstractions and recommendations in a guideline by means of a generic rule or a procedural language. Nevertheless, these formalisms still aim for generality in being able to encode all kinds of guidelines. Therefore, this formalism also has to use abstractions, such as actions, decisions, and criteria, as their primitives. Clinicians and knowledge engineers still have to conceptualise a guideline in these abstract terms. Research in the 1980s has shown the power of domain-specific models. For example, OPAL, a domain-specific tool for acquiring cancer chemotherapy protocols, is adequate for being used by clinicians [33]. Peleg and Tu [34] suggest to support the translation of narrative guidelines by providing computer interpretable templates for representing guideline knowledge using clinical abstractions that are appropriate for particular guideline sub-domains. These templates (also called design patterns) provide domain experts with abstractions that are defined at the same level as that of their guidelines. Screening and immunization templates have the potential to be applied to encode any guideline belonging to these categories. This was demonstrated by their ability to encode all of the screening guidelines from the National Guideline Clearinghouse that contained a clinical algorithm and all of the CDC immunization guidelines. In addition, knowledge modelled in the immunization patterns (1) can be checked for completeness and consistency, (2) could be used by applications that execute workflow processes or that compute the due date of the next vaccination, and (3) could be automatically translated into a computer interpretable guideline.

\subsubsection{Document-centric approach}

Below, we discuss the document-based approach that uses the languages HTML and XML, and allows clinicians to browse through documents in an efficient manner. We provide a brief overview of this development and give some examples of the approach.

The first step is using relevant tags in the mark-up process. The result can be translated in one of the languages of the guideline models. Shiffman et al. [16] describe such a document-centric approach. They use the Guideline Elements Model (GEM) as a computable representation for clinical guidelines. GEM is an XML-based document 
model that uses a multilevel hierarchy to store the heterogeneous kinds of information contained in clinical practice guidelines. The hierarchy contains more than 100 tags by which guideline information can be classified (marked up) and modelled at varying levels of abstraction. In the mark-up process the user selects text from the guideline and places this text in the appropriate position in the GEM hierarchy. In this way an XML file is produced. The text is specified further by removing unnecessary words, changing verb phrases from passive to active voice, etc. Also, abstract recommendations are made clear and ambiguity is removed.

In the second development step, the text is translated into computable statements. The results have been used to obtain a description in the Arden syntax. Moreover, the possibility of translating the mark-up results into GLIF3 (a guideline modelling language) by defining elements that represent GLIF constructs is investigated. The XML representation format used by GEM could limit its potential impact, as semantic web ontology languages, such as OWL, are becoming major knowledge representation frameworks in medical informatics. Nam Tran et al. [35] present a faithful translation of GEM from XML into OWL.

Next to GEM we can find more tools that support guideline implementation. We present here three of them.

Votruba et al. developed the Guideline Markup Tool (GMT) that not only supports the translation of clinical guidelines into a guideline modelling language such as Asbru but also maintains the connection between the original guideline and its formal representation [36].

The Stepper tool was developed to assist a knowledge engineer in developing a computable version of narrative guidelines. The system is document-centric: it formalises the initial text in multiple user-definable steps. They correspond to interactive XML transformations [37].

Shahar et al. [38] introduced a Web-based, modular, distributed architecture, the Digital Electronic Guideline Library (DeGeL), which facilitates gradual conversion of clinical guidelines from text to a formal representation in a chosen target guideline ontology. Their guiding principle is that expert physicians should be transforming free-text guidelines into intermediate, semantically meaningful representations, while knowledge engineers should be converting these intermediate representations into a formal executable representation. Shahar et al. demonstrated the feasibility of the architecture and the tools for several guideline ontologies, including Asbru and GEM.

\subsubsection{The bridging approach}

Having developed many guidelines and facing even more recommendations, we may investigate the relation between both. Shankar et al. [4] report that in a survey users expressed the need for a presentation of the text of the guideline together with the 
recommendations. However, both the knowledge-based approach and the documentcentric approach have difficulties in integrating guideline text with decision-support systems. Therefore, Shankar et al. proposed a bridging approach for integrating structured knowledge bases with marked-up text. They introduced clinical queries that might be posed by a clinician to gain better insight into guideline recommendations. The elements in the knowledge base are then linked to the appropriate clinical queries. Subsequently, an information retrieval technology is used to satisfy the queries by retrieving relevant excerpts of the guideline document.

\subsubsection{Implementation in Decision Support Systems}

In the course of our research, it is important to examine to what extent the guidelines and the implementation in decision support systems influence the users.

To adapt the clinicians' behaviour, CPGs should provide adequate patient-specific decision support during patient encounters, which is reliable and understandable by the clinicians. Guidelines implemented in a Computer Interpretable Guideline (CIG) formalism providing support based on patient data may achieve this goal. Overtime, we observed an increase of guidelines implemented in Decision Support Systems (DSSs) [39-41], where the system helps users to follow the guideline. In a DSS CIGs are implemented in a formalised way. The DSS retrieves all necessary patient data from an EPR and generates patient specific advice based on these patient data and the guideline content.

DSS cover more than only guidelines. Alerts and reminders, however, can be considered as simple guidelines. What can be said on the reasons for using or not using DSSs in general, is in our opinion, also valid for guideline implementation systems. In a systematic review, Kawamoto et al. [42] identified four features of DSSs that were critical for improving clinical practice: (1) decision support is provided automatically as part of clinical workflow, (2) decision support is delivered at the same time and location of decision making, (3) actionable recommendations are provided and (4) DSSs are computer-based. A common theme of all four features is that they make it easier for clinicians to use such a system: the system must minimise the effort required by clinicians to receive and act on system recommendations. The success of a DSS may concern the process or patient outcome or both. In a recent systematic review Mollon et al. [43] concluded that the majority of 41 reviewed studies concerning DSS for prescription reported changes in healthcare provider behaviour (in 25 of the 37 successfully implemented systems), but that only five studies showed a successful impact on patient outcomes. DSSs may also provide a better overview of existing, implemented guidelines.

In 2008, Wright and Sittig [44] reviewed the history of clinical decision support and traced the development of architectures for clinical decision support systems. They arrived at four distinct architectural phases for decision support: (1) stand alone decision support 
systems, (2) integrated systems, (3) standards-based systems, and (4) service models. Furthermore they developed a model for evaluating architectures for clinical decision support that focuses on (1) defining a set of desirable features for a decision support architecture; (2) building a proof-of-concept prototype; (3) demonstrating that the architecture is useful by showing that it can be integrated with existing decision support systems and comparing its coverage to that of other architectures [45].

\subsection{Computer Interpretable Guidelines}

40

In this section we discuss the knowledge-based approach to the creation of CIGs. In subsection 2.5.1 we describe the general guideline model and distinguish the underlying model and the language in which the guidelines are specified. In subsection 2.5.2 we discuss the representation in combination with its implementations. In subsection 2.5.3 we provide an overview of the problems encountered while designing (or using) the CIGs.

\subsubsection{General models}

It is possible to define the functionality of CIG approaches in terms of two main characteristics: (a) the underlying model, i.e., the framework of the logical components (2.5.1.1) and (b) the language in which guidelines are specified (2.5.1.2). We conclude by providing several approaches (2.5.1.3).

\subsubsection{Logical components}

The underlying model is the core characteristic of every guideline approach. It must be able to represent various kinds of guidelines that differ considerably in complexity and in level of abstraction. Two telling examples are nesting and decomposition. The model must contain a set of building blocks (primitives) used to construct guidelines, such as tasks, rules, nodes, and frames. Most approaches model guidelines in terms of a tasknetwork model, a (hierarchical) model of the guideline control-flow as a network of specific tasks (such as decisions, actions or hierarchically decomposed sub-guidelines). The control-flow part of the model should allow tasks to be executed sequentially or in parallel, allow cyclical or iterative tasks and permit entry points into the guidelines.

\subsubsection{Formal language and execution engine}

The guideline model should be supported by a formal language which specifies the guidelines in terms of the above-mentioned model primitives. Usually, such a language consists of two parts: a control-flow language and an expression language. The controlflow language specifies the guideline structure in terms of primitives of the model and 
their (temporal) relations, whereas the expression language usually describes decision criteria. GLIF for example used an expression language called GEL, based on the Arden Syntax's logic grammar. Later, GELLO, an object-oriented expression language, was developed. GELLO is better suited for GLIF's object-oriented data model, is extensible, and allows implementation of expressions that are not supported by the Arden Syntax. Patel et al. [46] compared the newest version of GLIF (GLIF3) with the previous one (GLIF2). GLIF2 (the first published version of GLIF, GLIF1 is only known as experimental version) supported guideline modelling as a flowchart of structured steps which represented clinical actions and decisions. However, the attributes of these constructs were defined as text strings that could not be parsed, preventing the resulting guidelines from being able to make inferences during computerised execution. Patel et al. analyzed the process of encoding guidelines in those two versions. They concluded that the use of GLIF3 appears to offer several improvements over GLIF2 in the ability to encode guidelines accurately and efficiently. The increased formality of the GLIF3 model and syntax leads to a guidelineencoding process that contains both a greater level of detail and less ambiguity than those in the previous version. The result of the improvements is an encoded guideline which is better equipped to aid practitioners as they make decisions in a variety of clinical settings. A formal language must be interpretable by automatic parsers. Preferably each approach should include a guideline execution engine incorporating such a parser that is able to provide decision support based on the encoded guidelines and patient data.

Wang et al. [47] describe the design and implementation of the GLIF3 guideline execution engine (GLEE). The paper gives a good overview of the internal structure of the execution engine. It explains how GLEE executes the various primitives of GLIF and illustrates the role of GEL, the expression language used to encode decision criteria and patient states.

\subsubsection{Modelling approaches}

Examples of guideline modelling approaches are: PROforma, GLIF, GUIDE, PRODIGY, EON, GASTON, and Asbru. Overviews of computer interpretable guideline formalisms were presented by de Clercq et al. [48], by Isern and Moreno [49], and by Sonnenberg and Hagerty [50]. Because of the available reviews on guideline modelling approaches, we will not cover this topic in depth. A good overview of methods for formalizing clinical practice guidelines can be found in Peleg et al. [51]. They compared six generic computer interpretable guideline models to determine their commonalities and differences.

Wang et al. [52] reviewed eleven guideline representation models and determined the primitives which these models use to represent guidelines. They discern action, decision, patient-state, and execution-state primitives. These primitives need to be organised to form a specific process model. Such a model also defines scheduling constraints on the primitives and nesting of guidelines during guideline application. Scheduling constraints 
specify the temporal order in which the primitives can be executed during guideline application. Nesting of guidelines defines the hierarchical relationship among guidelines. Patient states can be used to record a patient's clinical status in a specific context of a guideline. These patient states can then be used as entry points into a guideline. In this way multistep clinical processes that may take place over several encounters can be modelled. With execution states, steps scheduled, e.g., by GLEE, can be distinguished from those actually executed according to a user's decision.

Seroussi et al. [53] described the framework used by the ASTI system to represent therapeutic strategies in such a way that any patient's therapeutic history can be located within a guideline concerning hypertension. Because chronic diseases as well as the patient's response to treatments evolve over time, new therapeutic decisions depend on decisions made and actions taken at previous consultations, as well as the outcomes of those actions. This makes it difficult to locate the position in the guideline where to continue. To make such a position easily accessible, the guideline knowledge is represented as a two-level decision tree, a clinical level describing theoretical clinical situations and a therapeutic level formalizing the different steps of corresponding recommended therapeutic strategies. The therapeutic level of the knowledge base represents the sequence of treatments recommended for each clinical situation described at the clinical level. We remark and regret that the framework is only used in the guiding mode of ASTI (see Bouaud [54]).

\subsubsection{Representation and implementation}

There is a large difference between the information contained in published guidelines and the knowledge and information that are necessary to implement them [51]. Implementers use poorly specified, mainly tacit knowledge-acquisition processes. Thus, such an implementation approach results in considerable inconsistencies in the encoding of guideline knowledge and in the functionality of the systems that are created [16]. For example, Patel et al. [24] found, that in some cases different recommendations would be given to the same patient when using computable representations of the same guidelines implemented by different individuals.

A second problem is the lack of standardization of EPRs. There are many vendors offering EPR systems. However, there are no standards yet that EPR software providers have to be compliant with. Guideline systems have to map the terms used in the guideline to the corresponding terms used in the EPR systems. Here we seem to face an analogous problem as above. Yet, in the last decade considerable progress has been made and standardized approaches for guideline representation and sharing are central to these efforts [55]. Among them are rule-based, logic-based, and task-based approaches. We discuss knowledge representation in 2.5.2.1, sharing the knowledge in 2.5.2.2, and data representation in 2.5.2.3. 


\subsubsection{Knowledge representation}

Below we discuss the rule- and task-based approach.

The Arden syntax is a rule-based formalism developed for encoding individual clinical rules as Medical Logic Modules (MLMs). MLMs do not provide full support for conceptualizing a multistep guideline that unfolds over time.

A number of other developments share a hierarchical decomposition of guidelines into networks of component tasks that unfold over time: the Task-Network Models (TNMs). Unlike rule-based systems, TNMs can explicitly model alternative pathways or sequences of tasks, and they provide tools for visual representation of plans and the organisation of tasks within them. All TNM languages decompose guidelines into networks of component tasks and express various arrangements of these components and interrelationships between them. The modelling formats use different terminology to refer to various types of task networks (called plans by Peleg et al. [51]) and their components. Peleg et al. note that authoring CIG models can be time-consuming and may require clinical knowledge as well as technical skill. Translating guidelines encoded in one format into systems using other formats would reduce duplication of effort. The GLIF project originally intended to devise an interchange format to facilitate this process. However, these goals appeared to be impractical at the time.

\subsubsection{Sharing the knowledge}

Many parties are developing computer-based guidelines as well as decision support systems that incorporate these guidelines. The resulting products show much redundancy and overlap and there is little standardization to facilitate sharing of technical modules or to enable adaptation to local practice settings. This situation limits the sharing of guidelines. In a computer environment, implementation involves a number of steps to translate the knowledge contained in guideline text into a computable format. There is the problem that the terminology used in CIGs is different from the terminology used in EPRs. Several publications report about approaches that target the above mentioned problems in different ways. Below we discuss eight of them.

(1) Boxwala et al. [17] discuss the problem of sharable CIGs. They mention three ways to share CIGs: (1) translation of the formats into the proprietary format of the receiving system, (2) sharing of $\mathrm{CIGs}$ as decision-support services provided through standard application programming interfaces adopted by all systems, and (3) adoption of a common format for CIGs. The first option has limitations because it is not clear that one format always can be translated into another format without problems. The second option has been successfully pursued. But also here, the problem is that relevant $\mathrm{CIGs}$ may be specified in different formats that may not be interpretable by the execution engine of the decision support service. Therefore, Boxwala et al. prefer 
the third option to provide requirements for a format for sharable CIGs.

(2) A model to share guidelines encoded in different formats at the execution level was developed by Wang et al. [56]. They extracted a set of generalised execution tasks from existing guideline representation models. Then mappings were created between specific guideline representation models and the set of generalised execution tasks. They also developed a generic task-scheduling model to harmonize the existing approaches to guideline scheduling. This Guideline Execution by Semantic Decomposition of Representation (GESDOR) model is able to execute guidelines encoded either in GLIF or in PROforma.

(3) Tu et al. [57, 58] started the SAGE (Standards-based sharable Active Guideline Environment) project and created a service model approach. They observed that more than one formalism, for medical logic, are needed to accomplish sharing of computable medical knowledge. Lack of standards in terminologies and in data models for patient information require re-coding of parts of the guideline. Reuse of a guideline knowledge base is possible once an infrastructure is in place that includes a medical record query interface, terminology mediation, and an act interface. SAGE places a standard application programming interface in front of a clinical system. This solves the vocabulary problem - the SAGE virtual medical record specifies the vocabularies that will be used to access and process the medical data. The SAGE system will respond to opportunities for decision support in the care process. SAGE does not require a detailed workflow model but uses an event-driven approach. Here we remark that many workflow contexts need to be modelled to recognize appropriate events that should trigger decision-support services. These contexts identify (1) opportunities for providing decision support, (2) the roles and information needs of care providers, (3) events that may activate the guideline system, and (4) the guideline knowledge relevant in these contexts. The major innovation of the SAGE Guideline Model was its demonstration that heterogeneous information sources, such as patient data, order sets, and external knowledge sources can be integrated and used within encoded guideline knowledge bases. The Clinical Information System (CIS) events encoded in the guideline are registered by the event manager in the CIS, thereby expressing the execution engine's interest in these CIS events. When a relevant event is detected, the engine starts the execution. The SAGE execution engine is able to execute the guideline by interpreting the encoded content, obtaining current patient data from the $\mathrm{CIS}$, and invoking functionality within the CIS to implement an action specified in the guideline. The engine interprets the content of the context, action, and decision nodes in an encoded guideline, executes workflow and decision logic, and interacts appropriately with the CIS. The event listener is the mechanism by which the engine is notified of state changes in the CIS. As part of conforming to the SAGE engine, the CIS implements the module that forwards events of interest to the event listener. 
(4) Fox et al. [59] noted the possibility to capture guidelines in a computer interpretable form, opening up the capability of using the internet to deliver patient-specific advice and other services. They described a development lifecycle and technology for publishing and delivering services at the point of care (called publets) and discussed the quality requirements.

(5) Kawamoto and Lobach proposed a service-oriented architecture (SOA) for DSSs. DSS capabilities were implemented through the orchestration of independent software services of which the interfaces were standardized. Core services included: a decision support service, a common terminology service, and a retrieve, locate, and update service [60]. They also developed a decision support Web service to address the difficulty of sharing medical knowledge in a machine-executable format based on this SOA approach: SEBASTIAN (System for Evidence-Based Advice through Simultaneous Translation with an Intelligent Agent across a Network) [61].

(6) To make computerised CPGs potentially accessible to a large number of general practitioners (GPS), the PRESGUID project [22] proposed an online service enabling physicians to consult computer-based CPGs. PRESGUID provides a web-based guideline system that takes as input clinical data on a particular patient and returns, as output, the customised recommendation. If the recommendations require prescribing drugs, the system will query the drug database and will display detailed information about the relevant specific medications.

(7) Dominguez et al. [62] reported about the creation of a traceable clinical guideline application based on model-driven development techniques, using UML state charts. The models could be shared and implemented using a plug-in. Their approach focused on models conforming to domain application metamodels, allowing code programs to be automatically generated from them by means of a refinement process. They also described the way in which the structure of the execution traces was automatically generated, represented not as a linear sequence but as a hierarchy of states, providing one straightforward and reliable way of obtaining complete storage structures of guideline applications. The complete framework has been implemented as an Eclipse plug-in named GBDSSGenerator. Different types of clinical guidelines were generated including laboratory guidelines. However, until now, each guideline is translated into a separate program.

(8) Skonetzki et al. [63] proposed a framework to represent clinical guidelines, called HELEN. It was not their intention to supersede the variety of proposed and implemented approaches for formal representation of clinical guidelines. Instead they focused on management and implementation specific topics to bring CPGs into clinical practice. The complexity of the authoring process was considered to be the real bottleneck. To achieve the goal of developing a flexible, shareable, and computable description of 
the CPG, they used ontologies created with the help of PROTÉGÉ [64]. They showed the benefits of combining different knowledge representations such as narrative text, graphic illustrations, and algorithms. They introduced a possible approach for an explicit adaptation process of documented and auditable CPGs in order to make them institutionspecific and support sharing with other organisations.

\subsubsection{Data representation}

For implementing guidelines in systems, data representation is an important aspect, of course next to knowledge representation. For instance, item names used in the guidelines are usually different from the item names used in EPRs. The representation of data should therefore be seriously considered in order to facilitate that guideline implementation systems can be easily integrated with EPR systems. Standardization is of utmost importance here. This was already acknowledged in the SAGE project described above.

Johnson et al. [65] discussed the use of a virtual medical record to solve the problem of idiosyncratic data models in the deployment environment. In this case, guidelines are encoded, assuming (1) a uniform virtual medical record and (2) guideline-specific concept ontologies. For implementing a guideline-based decision support system in multiple deployment environments Johnson et al. created mapping knowledge bases to link terms in the concept ontology with the terminology used in the deployment system.

\subsubsection{Validation and verification of computer interpretable guidelines}

When translating CPGs to CIGs inconsistencies, ambiguities, and incompleteness can be detected in the paper-based guideline. Therefore, an adequate translation by a guideline modelling language can increase the quality of guidelines. This was, among others, reported by Marcos et al. [66]. They tried to answer the question whether formalization could improve the quality of medical protocols. Their line of reasoning was as follows. Protocols show diversity in formats, e.g., texts, flow diagrams, and tables. Making these protocols more precise with the help of a formal language may reveal anomalies in the guideline description. In their work, they translated two protocols into a computer-based language, Asbru. During protocol formalization into the Asbru language, they found a number of anomalies in the guideline, such as ambiguity, incompleteness, inconsistency, and redundancy. The language sometimes demands explicit elements which are not explicit in the original protocol. Sometimes Asbru required information that otherwise would go unnoticed. An example is the minimal and maximal delay necessary for the specification of the retry time in a cyclical plan. The authors concluded that (1) removal of anomalies increased the internal consistency of the protocol, (2) formalization is a good foundation for detection of anomalies, and (3) formalization of the protocol is intensive 
and costly. In section 2.8 we mention a number of formal methods that can be used for testing whether $\mathrm{CIGs}$ conform to their specifications.

Formalization can easily spot protocol aspects such as the specificity and certainty of recommendations that are stressed by the AGREE appraisal instrument. The strength of formalization when compared with other methods lies in the use of precise notions.

\subsection{Effects of guidelines}

Many groups are working on the development and implementation of guidelines. It is interesting to know whether guidelines produce any significant changes. CPGs may have potential benefits (see (a) below) but also limitations (see (b) below) for patients, physicians, and the healthcare system (see Woolf et al. [28]).

(a) Patients may profit from CPGs that summarise the benefits and harms of available options. Patients can make more informed healthcare choices taking their personal preferences into account. Also, services which were not offered to the patient earlier may become available as a response to newly released guidelines.

Moreover, the healthcare system could profit from improved efficiency (standardized procedures) which can lower the costs of services, drug prescriptions [67], or (3) laboratory test requests $[68,69]$.

(b) In contrast to the above, patients may also be harmed by clinical guidelines. Cost of healthcare may influence the design of new guidelines and patients' individual needs may not be the only priority in making recommendations. Groups developing clinical practice guidelines need to be aware that what is best for patients overall, as recommended in the guideline, may be inappropriate for individuals.

Below we discuss the effect of using guidelines, both CPGs and CIGs, on the process of care (2.6.1) and on the patient outcome (2.6.2).

\subsubsection{Effect on the process of care}

Many groups focus their work on finding out whether CPGs or CIGs do make a difference, and whether they harm or benefit the process of care. In 2.6.1.1 we review three cases described in the literature that deal with above mentioned issue and in 2.6.1.2 nine cases that deal with CIGs.

\subsubsection{Clinical practice guidelines}

In a recent study on the effects of evidence-based CPGs on quality of care in the Netherlands, Lugtenberg et al. [70] stated that there are positive indications for the effectiveness of Dutch evidence-based CPGs on the structure and process of care. The 
size of the effects varied largely across recommendations within the guidelines.

Butzlaff et al. [71] investigated in a randomised controlled trial whether access to four web-based and evidence-based guidelines (not computer interpretable) increased the knowledge of the general practitioners (GPs) using the guidelines. No statistically significant knowledge increase was observed, but the authors did observe a low usage of the guidelines in the intervention phase. They assumed that the number of implemented guidelines (only 4) and the rather short time of intervention ( 2.5 month) may have played a role. Physicians explained that their hesitation to use the web was caused by the concern that patient data in their computer unintentionally and illegally might become available to hackers during their on-line time. Their second main reason for not using web-based guidelines mentioned by the physicians was "lack of time".

Jousimaa et al. [72] observed no difference in guideline use or in impact on decision making between an intervention group of newly qualified primary care physicians that had access to an electronic narrative version of guidelines and a control group of similar physicians having access to the paper version. In this study, textbook and computer-based guidelines were frequently used and were the most common source of information. The authors observed that the implementation of computer-based guidelines may need more training and investment in computer hardware. However, once computers are readily available and routinely used within consultations, the computer-based version offers many advantages, such as easy updating, low production costs, possibility to include other databases and audiovisual material, the possibility of linking computer-based guidelines to decision support systems, and the ability to monitor the guideline use.

\subsubsection{Computer interpretable guidelines}

In their systematic review, Shiffman et al. [73] looked at the functionality and effectiveness of computer-based guideline systems. They assessed the functionality and effectiveness of DSS and checked which of the eight services (see below) from their information management services model were implemented [74]. This services model was hypothesized to contain the factors that would influence the success of guideline implementations. The model facilitates workflow integration. It considers eight factors: recommendation, documentation, explanation, presentation, registration, communication, calculation, and aggregation. They found that 14 of the 18 described cases mentioned improvement in guideline adherence, although they could not prove that the presence of the mentioned factors was predictive for the success of the studied systems. To evaluate adequately the effect of those factors on the success or failure of a computer-based guideline implementation, more of the confounding variables (such as different types of guidelines, different settings, and different system implementations) need to be controlled. 
Carton et al. [69] reported about a study where, concurrently to the care, the computer provided personalised advice for a patient. The authors found that only seven common clinical situations were responsible for the majority of unnecessary radiological requests (70\%). When compared to the control situation in which no recommendations were provided, a decrease of $6 \%$ of requests not conforming to the guideline was observed. Yet, the overall effect of the intervention concerning radiological requests appeared to be weak. It became visible that in these situations the theoretical knowledge of junior practitioners was inadequate and additional education could provide the solution.

Hunt et al. [75] reviewed studies on the use of DSSs in clinical settings, a subject related to guideline implementation. A total of 68 controlled trials met their inclusion criteria. 65 studies assessed physician performance (in $66 \%$ a benefit was found) while 14 studies assessed patient outcomes (in 6 cases a benefit was found).

In a follow-up study six years later Garg et al. [76] found that $64 \%$ of the studies on physician performance reported that the DSSs increased performance and $13 \%$ of the studies regarding patient outcomes established a benefit of DSSs. They concluded that DSS can considerably enhance, for example, clinical performance for drug dosing and preventive care, but not so much for diagnosis. They also concluded that the practitioners' performance in many cases did improve, but that more research is needed to explain the effects of such systems on patient health. Garg et al. raise an important question: to what extent should DSSs be proven beneficial before mass deployment?

Ambresin et al. [77] built a study website for the management of febrile patients returning from the tropics. The site was built to provide medical diagnostic assistance to primary care physicians. Physicians can interact with the computer in real-time. The authors called the study website a success. The interest of physicians in tropical diseases and evidence-based medicine increased, and therefore their knowledge increased as well. There are still problems which they need to address. For example, the observation that only $50 \%$ of the recommendations were followed, might be caused by approaches that are inappropriate or not feasible. Thus, the system needs revision and an update of the guideline before being adopted by the end-user. If that is achieved, an improvement of the quality of care is expected.

In their work Overhage et al. [78] point out that physicians often fail to order tests or treatments needed to monitor/ameliorate the effects of other tests or treatments (corollary orders). They wanted to see whether automated, guideline-based reminders to physicians, provided when they wrote orders, could reduce these errors of omission. To test whether guideline-based reminders could help, they carried out their study in the inpatient general medicine wards (six independent services) of a teaching hospital. They identified 87 target orders that could be paired with one or more corollary orders. The rule-based reminder program analyzed the data in the EPR for the omission of any 
corollary orders and presented these to the intervention group physicians (belonging to three of the six services). They concluded that computer suggestions about corollary orders had a large effect on the adherence to the guidelines, especially when measured in terms of immediate or 24-hour compliance. The interventions increased adherence to many guidelines that were being promoted by the Pharmacy and Therapeutic Committee. Pharmacists had to call physicians to ask about drug-related interventions one third less often for study patients than for control patients. They did not observe any effects on patient outcomes, such as length of stay, serum creatinine, or charges. However, since the guidelines handled only $9.6 \%$ of the orders written during this study, it was not expected to occur.

Bouaud et al. [54] noted that onscreen reminders and alerts automatically provided by a DSS do not guarantee a positive impact on a physicians' compliance with recommendations. They hypothesized that two situations can be distinguished in primary care that have a different impact on the way decision support will be used. On the one side there are cases that the GPs know how to solve although they may make mistakes. These are usually relatively easy cases for which reminder-based interaction to detect these mistakes is mandatory. On the other side there are hard cases for which GPs may seek up-to-date evidence to support decision making. In such cases GPs would deliberately use guidance systems (the available knowledge has to be browsed by the GP). The authors used ASTI, a prototype guideline-based DSS to test their hypothesis. ASTI has two modalities: a critiquing mode and a guiding mode. In the critiquing mode recommendations are automatically generated based on the patient data. In the guiding mode the knowledge base is not automatically processed but can be read by the GP using browsing tools. Bouaud et al. concluded that the critiquing mode was more used for easy cases and that the guiding mode was more used for medium and high complexity cases (the hard cases).

In their work Goud et al. [79] observed a positive impact on the professional's adherence to the cardiac rehabilitation guidelines when using a DSS. CARDSS (Cardiac Rehabilitation Decision Support System) did improve users' familiarity with the guideline's recommendations and decision logic, and reduced guideline complexity by supporting calculations and interpretations of data. Goud et al. also observed that if the system recommendations were presented to patients, refusal to participate in therapies reduced. They concluded that a computerised decision support system can be effective in improving a multidisciplinary team's guideline concordance [80].

Chan et al. [81] warned that the measurement of provider adherence to determine the effect of CIGs may provide a too pessimistic view with respect to patient outcome. Because many factors next to decision support determine patient morbidity and mortality, it would be difficult to measure accurately the effect of a DSS on such outcomes. Evaluation of 
provider adherence to a particular recommendation made by the DSS is the most direct indicator of the ability of a DSS to affect clinical decision-making. It was used to evaluate the effect of CIGs. In this approach Chan et al. distinguished between strict adherence and adherence in the spirit of the guideline. They analyzed provider adherence to the ATHENA DSS recommendations. From the results presented we can see that the providers strictly adhere to, e.g., a substitution (of drugs) advice only in $1.2 \%$ of the cases, whereas adherence in the spirit of the guideline was much higher: $37.5 \%$. Therefore, Chan et al. concluded that a strict evaluation of actions and recommendations had failed to describe in full detail the providers' adherence to the guideline. Any valid measure of adherence must consider the extent to which the provider strictly follows the guideline and the provider's more clinically relevant higher-level intention.

\subsubsection{Effect on the patient outcome}

It is interesting to investigate whether (1) guidelines can cause changes in the physicians' behaviour and (2) improve the process of care, and simultaneously whether there is any effect on the outcome such as patient morbidity and mortality. We discuss those questions for CPG (2.6.2.1) and CIG (2.6.2.2).

\subsubsection{Clinical practice guidelines}

In their work, Lugtenberg et al. [70] observed changes in patient outcomes. Yet, they concluded that they were generally modest and did hold only for some of the outcomes that were studied. Lugtenberg et al. suggested that guideline implementation should focus more on individual recommendations than on the guideline as a whole.

Worrall et al. [82] assessed the evidence for the effectiveness of clinical practice guidelines in improving patient outcomes in primary care. They identified 13 trials that examined patient outcomes. Four of the studies followed nationally developed guidelines and nine followed locally developed ones. Six of the studies involved computer-based or automated reminder systems. Only five studies reported statistically significant results. However, the lack of evidence of the effectiveness of the guidelines may well be due to the lack of methodologically sound studies among the conducted ones. Also, many of the studies that were evaluated, even those that were methodologically sound, may have examined guidelines that were based on out-of-date consensus statements or may have been poorly implemented.

Eagle et al. [83] described a project that applied guidelines in 10 hospitals in Michigan, USA and concluded that the application of guidelines improved the delivery of evidencebased care (mortality reduced at one year by $5.1 \%$ ). 


\subsubsection{Computer-interpretable guidelines}

Heselmans et al. [84] analyzed in a systematic review the effectiveness of computer interpretable guidelines implemented in ambulatory care settings. They concluded that patient outcomes were not widely studied. No evidence was found of an effect on patient outcome. They also found that only seven of seventeen studies that investigated process outcomes showed improvements in process of care variables compared with the usual care. No incremental effect was found of the electronic implementation over the distribution of paper versions of the guideline, neither for the patient outcomes nor for the process outcomes. They concluded that there is little evidence for the effectiveness of electronic guidelines. The results by Heselmans' et al. were less positive than those of the other studies mentioned. They explained the difference by noting that in their study the more effective straightforward reminder systems were excluded. Also, the definition of a successful intervention may have played a role.

Bryan et al. [85] evaluated the types and effectiveness of electronic DSSs in the primary care setting. They concluded that a DSS has the potential to produce statistically significant improvements in outcome, although there is much variability among the types and methods of DSS implementation and resulting effectiveness.

\subsubsection{Section conclusions}

By reviewing studies that focus on the effects of guidelines on the process of care, we observe that guidelines can positively influence the work, improve guideline adherence, and increase efficiency (e.g., test ordering).

We notice that there are much less studies that focus on the patient outcomes. We need to bear in mind that since many factors, next to decision support, determine patient morbidity and mortality, it is difficult to measure accurately the effect of a DSS on such outcomes.

\subsection{Barriers to guideline adherence}

From studies and reviews we may conclude that the adherence by physicians to guidelines is low both for paper-based and computer-based guidelines. However, the problems causing this noncompliance are different in both cases. We have guideline - related barriers (2.7.1) and other barriers (2.7.2).

In case of narrative guidelines, physicians' low adherence is conditioned, for example, by lack of awareness that the guideline exists, lack of time to look up the answer especially in urgent situations, or vagueness of recommendations. In case of computerbased guidelines the physicians' adherence is related to workflow integration, general 
attitude towards computer systems, and agreement with the content of the guideline that underlies the knowledge base.

We discuss these barriers and in 2.7.3 we concentrate on improvement. Finally, in 2.7.4 we deal with the question: has guideline development gone astray?

\subsubsection{Guideline-related barriers}

Already Grimshaw et al. [86] concluded that clinical practice guidelines increase quality and outcome of care. In their review they studied 59 published evaluations of clinical practice guidelines. They point out that the successful introduction of clinical practice guidelines depends heavily on the used methods of development, dissemination, and implementation. Different methods will be appropriate in different contexts. Studies that report large improvements in clinical care suggest the potential of guidelines when development, dissemination, and implementation are all appropriate. Studies that report small improvements or none may reflect failure at any stage during the introduction or evaluation of the guidelines. They may also reveal barriers. Grimshaw et al. presented a classification highlighting the more effective strategies and suggested that the strategies most likely to be effective were those that were internally developed, where the dissemination strategy was a specific educational intervention and where patientspecific advice was delivered concurrent with care. Having these characteristics may help considerably to overcome the guideline related barriers.

Over time, guidelines were disseminated in many forms. They are published in magazines and journals, textbooks, CD-ROMs, and now also on the Web. While electronic dissemination has broadened the availability of guidelines and enables guidelines to be retrieved in clinical settings, most guidelines have typically been specified in a noncomputer interpretable narrative text or in non-executable flowchart formats. These noncomputable formats limit the usability of the guideline since the knowledge contained in the guideline may not be easily accessible during the patient encounter. This is a main limitation. Further, extracting recommendations from a non-computable document and determining their relevance for a specific patient require additional effort from the care provider. The usability issues mentioned have been identified as factors impeding compliance with the guideline [87].

Shaneyfelt and Centor [7] point to another limitation of guidelines: they are often focused too narrowly on single diseases and are not patient focused. Patients seldom have single diseases and few guidelines help clinicians in managing complexity.

\subsubsection{Other barriers to physician adherence}

Other barriers exist too. Guideline adherence among Dutch general practitioners, for example, is not optimal [88]. An analysis of barriers to implementation was carried out 
by focusing on key recommendations rather than on guidelines as a whole. The barriers varied largely within guidelines (each key recommendation had its own barrier pattern). The most perceived barriers were lack of agreement with the recommendations due to lack of applicability or lack of evidence (68\% of key recommendations), environmental factors such as organisational constraints (52\%), lack of knowledge regarding the guideline recommendations (46\%), and guideline factors such as unclear or ambiguous guideline recommendations (43\%). To obtain more insight into these barriers we briefly discuss five studies.

Morris [23] in his work mentions the following eight barriers to protocol use: (1) lack of appreciation of the limitation of human decision making and the small number of variables on which decisions depend; (2) exclusion of practitioners from the protocol development process; (3) tendency to focus on infrequent but possible clinical scenarios that are not accommodated by protocol logic; (4) use of guidelines primarily for purposes other than improving quality of care and patient outcomes (e.g., reducing costs); (5) hubris among clinicians defending their autonomy; (6) concern that protocol logic is not correct; (7) concern about a reduced role of clinicians in medical practice; (8) insufficient technological infrastructure (no functional electronic patient records).

Cabana et al. [87] concentrate their work on finding reasons why physicians do not follow clinical practice guidelines. They investigated 76 articles and report seven general categories of barriers to physicians' guideline adherence. The barriers concern the physician's knowledge (lack of awareness, lack of familiarity), attitudes (lack of agreement, lack of self-efficacy) and behaviour (external barriers, guideline factors, environmental factors). However, they do not point out which barriers are the most common and need to be taken into account in the first place while creating clinical practice guidelines and building decision support systems. Also, they concentrate on the guideline as a whole, whereas Lugtenberg et al. [88] suggest focusing on individual key recommendations.

Trivedi et al. [27] also looked at barriers in physician's acceptance of computerised decision support systems. They divided those barriers into three groups: (1) human issues, (2) organisational issues, and (3) technical issues. They extended the list of known barriers by a few, such as: physicians' knowledge of and experience with computers, privacy concerns, the security of text notes, depersonalization of care, and losing eye contact with patients. Physicians should receive additional time and support to facilitate a structured data entry that improves quality and enhances effective clinical operations. Software needs to be flexible, e.g., is the program easy to log-on to and capable of providing for physician episodic sessions, commonly interrupted due to patient care. In accordance with Shiffman et al. [15] Trivedi et al. concluded that the importance of integrating DSS into the clinical activities and workflow is a prerequisite to overcome the other barriers. 
Espeland and Baerheim [89] identified and described factors that effected decisions about ordering plain radiography for back pain by general practitioners. Here, barriers to guideline adherence suggested by such factors may play a part. In addition four barriers other than those reported by Cabana et al. [87] were found: one concerning attitude (lack of expectancy that guideline adherence will lead to the desired healthcare process), one feeling-related (emotional difficulty with adherence), and two external (improper access to actual/alternative healthcare services, and pressure from other healthcare providers/organisations). Espeland and Baerheim concluded that their findings may provide a better understanding of how GPs try to achieve acceptable solutions in the face of conflicting pressure and uncertainty. Their findings may help to implement spine radiography guidelines by reducing the multiple barriers to change. Their review indicated that change can be achieved by addressing at least two of the following three types of factors: (1) predisposing (knowledge, attitudes), (2) enabling (skills, resources, reduction of external barriers), and (3) reinforcing (reward through feedback).

Tehrani et al. [90] wrote an overview of different methodologies used in various intelligent DSSs for mechanical ventilation. They tried to find the reasons for the infrequent use of DSSs in mechanical ventilation especially because according to the authors, mechanical ventilators will become more advanced and there is an increasing number of DSSs for mechanical ventilation. They compared different rule- and model-based techniques. Tehrani et al. concluded that the infrequent use of DSSs in mechanical ventilation is caused by: (1) lack of accessibility, (2) the system is not immune to noise and erroneous data, (3) inadequate training to use the system, (4) lack of implementation in commercial ventilators.

\subsubsection{How to improve guideline adherence?}

Several instances of implementation failure have been described. In many cases factors extrinsic to the guideline itself, e.g., organisational and provider-specific obstacles inherent in a particular system of care interfered with the implementation success. In other cases factors intrinsic to the guideline have contributed to implementation failure, e.g., ambiguity, inconsistency, and incompleteness. These three factors can in many cases be ameliorated or fully remedied by guideline authors while the guideline is being developed. During the translation of a narrative clinical practice guideline into a CIG, errors can occur. Peleg et al. [32] started a study investigating the translation process from a narrative guideline to a clinical algorithm. They studied this process by looking at intermediate versions produced during algorithm creation. They identified the types of errors introduced during the development process. The classification scheme proposed by Knuth was used to classify modifications between narrative guideline text and the clinical algorithm produced from it. Knuth classified discrepancies between the requirement 
document for TeX and the resulting software. The first 12 of the 15 modification types are also applicable to the narrative guideline domain. They recorded modifications made during several translation steps and recorded if it concerned a positive or negative change; whether it was an important one or not; and the location of the change in the paperbased guideline. They found that much of the information that was relevant for creating an algorithm had its origin in the medical background component of the guideline and not in the recommendation component. They concluded that when a clinical expert works alone to create an algorithm, contrary to the theory of "learning by doing", he is likely going to make more errors than positive modifications. Collaborative work is likely to result in much broader improvements to the algorithm. Finally, team work seems to be the best solution for detecting errors. They notify that a physician who is presented with a clinical guideline is going to interpret it based on his experience. Thus building a shared model may bring conflicts as every individual brings different knowledge and experience to the task. Depending on the group which is creating the guideline the final results may be different. That is why guidelines which are supposed to be broadly used have to be more universal, easy to interpret, and flexible.

Ambiguity and vagueness in clinical practice guidelines reduce the likelihood of clinician adherence according to Codish et al. [10]. Use of ambiguous and vague terms hampers communication and leads to uncertainty and to variable interpretation. The authors propose a model for ambiguity and vagueness in guideline recommendations. Their goal was (1) to provide guidance to guideline authors to enable them to reduce inadvertent use of ambiguous and vague language, (2) to improve transparency when vague language is used on purpose, (3) to create a framework to develop tools to apply the model during authoring and implementation of clinical practice guidelines. They propose a model containing three axes: the first axis differentiates vagueness from ambiguity and classifies each of them, the second axis indicates the intent of the author using vague or ambiguous terms, and the third axis defines what recommendation component of the guideline is affected by ambiguity or vagueness. The model can be used to develop software tools that are to be employed during the authoring and implementing process to identify and classify vague terms, inform authors of numerical values associated with the term, display an ordinal scale of similar terms, and suggest alternatives.

Grol et al. [91] determined attributes of clinical practice guidelines that influenced the use of guidelines in decision making in clinical practice. They concluded that evidence-based recommendations are better followed in practice than recommendations not based on scientific evidence. Also, precise definitions of recommended performance improved the use of guidelines. Testing the feasibility and acceptability of clinical practice guidelines among the target group is important for effective implementation.

LaBresh [92] described a program for quality improvement of acute stroke care. The 
program focuses on three domains: (1) diagnosing barriers to the delivery of care, (2) the clinical care system redesign process, and (3) a collaborative model. They mention knowledge, attitudinal and behaviour barriers. Knowledge of clinical trial results and guideline recommendations are a necessary prerequisite for delivering evidence-based care. Traditional physician education has focused on presenting this evidence. Too often, however, the dissemination of guidelines was assumed to be sufficient to produce high levels of adherence. Yet, even with the knowledge and intention to deliver evidencebased care, the performance still might not be optimal because of organisational barriers, in particular primarily lack of well-designed systems to ensure reliable delivery of desired care. According to LaBresh the development of highly reliable systems requires a team approach. They presented a collaborative model (a group of participating ("collaborating") healthcare delivery organisations studying a specific healthcare quality problem, designing and implementing specific solutions, evaluating and refining these solutions, and disseminating findings to the other organisations) for quality improvement.

Fieschi et al. [93] looked into the development of DSSs over time. According to Fieschi et al., the factors that influence the adoption of DSSs in today's medicine are: (1) 'evidencebased' practice is 'disease-' rather than 'patient-' oriented, (2) clinical practice guidelines while used in daily practice need to guide the decision-making rather than force the decision, (3) collaborative medical practice is the means of improving the quality, continuity, and coordination of care, (4) the possibility of shared decision-making, where DSS helps with, e.g., individual risk assessment or drug interactions.

Clinicians' adherence to DSSs is related to workflow integration [73], a general attitude towards DSSs and agreement with the content of the guideline that underlies the knowledge base [80]. This means that decision support needs to be provided at the right time and the right location, and the content needs to be trusted. This is precisely why guideline development by multidisciplinary teams, where medical professionals and system developers work together, is so important [29, 31, 32].

\subsubsection{Has guideline development gone astray?}

The quality of CIGs is clearly dependent on the quality of CPGs. As we have seen Tricoci et al. [6] concluded that updated guidelines contain more recommendations but many of these are based on lower-level evidence. In the British Medical Journal (BMJ) recently the question was raised whether guideline development has gone astray. Gibbons et al. [94] answered this question negatively. They pointed out that guideline development in cardiovascular diseases is a well developed process that has enhanced the delivery of proved treatments and improved patient outcomes. They illustrated their opinion with evidence proving that the implementation of practice guidelines led to positive changes in clinical practice. 
In contrast, Grol [95] answered the question positively. He stated that guidelines need to be integrated with other quality improvement initiatives, such as performance measurement and quality improvement programs. Guideline developers usually have quite different aims and interests from those in the quality improvement world. Guideline developers may have close ties with the industry. Many guidelines even today do not meet the AGREE criteria. Audits around the world show that guidelines are on average used in only $60-70 \%$ of day-to-day decisions. The cost effectiveness of guidelines compared with other methods for improving patient care is unknown. According to Grol procedures must be changed to speed up the development, minimise personal bias in recommendations and involve patients more actively in the process of both developing and using guidelines. Without such changes, guideline development may increasingly be seen as an expensive but unhelpful and ineffective toy for a happy few.

Also, in the medical informatics community it became clear that clinical decision support (CDS) implementation and use have been problematic. As a result, relevant medical knowledge that should be brought to bear is not always available or used for many healthcare decisions. On the request of the Office of the National Coordinator for Health Information Technology in the US, AMIA, the American Medical Informatics Association, established the CDS Roadmap Development Steering Committee to develop a tactical plan to guide federal and private sector activities to advance CDS. The goal of the Roadmap is to realise the vision of a U.S. healthcare system in which optimal, usable and effective clinical decision support is widely available to providers, patients, and individuals where and when they need it to make healthcare decisions.

The resulting roadmap [96] identified three pillars for fully realizing the promise of CDS: (1) make the best knowledge available when needed, (2) ensure high adoption and effective use of CDS and (3) continually improve knowledge and CDS methods. Each pillar comprises two strategic objectives. The best knowledge can be made available when needed when (1a) clinical knowledge and CDS interventions are represented in standardized formats and when (1b) clinical knowledge and CDS interventions are collected, organised, and distributed in one or more services from which users can readily find the specific material they need. High adoption and effective use can be reached when (2a) policy, legal, and financial barriers are addressed and when (2b) clinical adoption and usage of CDS interventions are improved in such a way that they are easy to deploy and use by identifying and disseminating best practices for CDS deployment. Continuous improvement of knowledge and CDS methods can be obtained by (3a) assessing and refining the national experience with DSSs and by $(3 b)$ advancing care-guiding knowledge by fully leveraging the data available in interoperable EPRs to enhance clinical knowledge and improve health management. 


\subsection{Discussion and conclusions}

Recent reviews show that decision support systems that issue alarms or reminders lead to a better care process. But still, it is not confirmed that the use of guideline systems that implement complex guidelines (1) improves the quality of healthcare, (2) lowers the medical costs of treatments, and (3) reduces practice variability. Below we provide our conclusions on: formalization of CIG (2.8.1), implementation (2.8.2), and effects (2.8.3) and add some discussion where appropriate.

\subsubsection{Formalization of Computer Interpretable Guidelines}

Formalization has many advantages but does not always work. We provide four conclusions based on our findings in the literature.

First, most guideline modelling approaches have facilities for testing that the model is unambiguous and syntactically correct [55].

Second, Marcos et al. [97] reversed the role of critiquing as used in some decision support systems. In such decision support systems the user solves a problem and the decision support system provides comments aimed at improving a suboptimal solution of the user. The critiquing approach assumes that the guideline is correctly implemented and that the contents of the guideline are error free. The critiquing procedure can also be used to test the output of the system and thereby the implemented guideline by comparing the actions proposed by the system to those of an expert. Marcos et al. compared the solutions of an expert physician to the recommendations provided by Asbru and analyzed the differences to improve the guideline. Marcos et al. tried manually to match the actions of the expert physician with those produced by Asbru. However, the expert's actions did not always appear in the protocol as plans and therefore there is no 1:1 correspondence between an expert's actions on the one hand and protocol plans and corresponding intentions on the other. The direct use of intentions for critiquing, either for matching of actions or for studying their appropriateness, was also not possible. This was a problem for the vocabulary, and frequently led to differences in the degree of detail, abstraction level, etc. Marcos et al. therefore concluded that using the critiquing approach for guideline improvement is not promising.

Third, an advantage of software systems is that formal methods can be used during their development to test whether they conform to specifications. Formal methods are mathematics-based techniques for the specification and verification of software and hardware systems. As medical errors have far reaching consequences, there is a good reason to investigate the usefulness of formal systems in medicine. Errors in guidelines may contribute to medical errors and mistakes. This may lead to unexpected harm to patients and to a low compliance of the use of guidelines. So, there is a growing interest 
to apply these methods carefully in the area of medical guidelines [98].

Fourth, given a specification that describes what the system should do, the formal verification is the act of proving or disproving the correctness of an implementation with respect to the specification in a mathematically rigorous way. Like mathematical instruments, formal methods can be used in a number of ways for guidelines. We mention three of them. (1) The guideline can be considered as a system that is being developed. Verification then involves checking whether this guideline adheres to certain correctness or quality criteria [99]. (2) National guidelines could be considered as the golden standard when developing a local protocol [100]. (3) Verification can also be used to identify possibly undesired behaviours (non-compliance) as well as to analyze and comprehend features and characteristics of the real process that are not properly expressed in the modelled process [101]. An application of this type of verification (e.g., model checking) is the following. Groot et al. [102] employ model checking to investigate whether a part of the actual treatment is consistent with the guideline. They proposed a computational method for such critiquing, where the ideal actions were given by a formal model of a clinical guideline, and where the actual actions were derived from real world patient data. Moreover, they showed how critiquing can be cast in terms of temporal logic, and what can be achieved by using model checking. The method has been applied to a clinical guideline of breast cancer in conjunction with breast cancer patient data.

\subsubsection{Implementation}

Guideline development and implementation is time-consuming and expensive. Developing high-quality guidelines requires a sufficiently skilled team of people and sufficient budget. Guideline development panels should include members with different expertise, including medical informatics. Our main conclusion here is that sharing of $\mathrm{CIGs}$ is a critical requirement for guideline development, dissemination, and implementation. The use of a universal standard for guideline representation to encode all guidelines would be a solution. However, since no existing guideline representation model is dominant over the others this approach is impractical today. Since there is much overlap between the primitives of the various models, a universal representation may be possible in the future. Moreover, the GESDOR approach showed that guidelines encoded in different languages could be executed by a generalised execution engine. So this engine could be used in order to be able to use guidelines represented in different models. Also, service-oriented architectures may support the prompt execution of guidelines. But here standardization of the interfaces as well as of the data is very important.

A paper-based and a semantically similar computer-based guideline should be designed simultaneously by groups of medical experts and knowledge engineers. This will make the step of the interpretation of a paper guideline superfluous. Because governmental 
agencies have larger resources than professional organisations and specialist societies and produce higher quality guidelines [12], they should take the lead in developing high quality $\mathrm{CIGs.}$

Guideline implementation systems obtain their data from EPRs. A standardized terminology should be imposed so that the terminology used in the guideline matches the terminology used in EPR systems. Service oriented approaches specifically take these problems into account.

\subsubsection{Effects}

Guidelines adherence depends on a variety of factors, some related to guidelines themselves, some related to users, and some to the implementation context. Among the former are guideline quality, purpose, and implementation modality. Among the user-related factors are attitude to behavioural changes, authority interventions to foster adherence and eventually the type of users (general practitioners, hospital professionals, home caregivers, patients, etc.). Context is also crucial because organisational issues, such as lack of resources, can hamper guideline implementation and sometimes the original guideline intention is overridden by the guideline adaptation to a certain setting. Quaglini [103] discussed these factors and highlighted them by presenting a number of case studies. She stressed the importance of determining the motivation for non-compliance. She presented a classification system for non-compliance that is used in RoMa, an ancillary tool for non-compliance management, coupled with a decision support system for stroke patients.

We end with two conclusions on effects.

(1) By reviewing studies that focus on effects of guidelines on the process of care, we may conclude that guidelines can positively influence the work, improve guideline adherence, and increase efficiency (e.g., test ordering).

(2) We conclude that there are much less studies that focus on the effects on outcomes. We need to bear in mind that since many factors, next to decision support, determine patient morbidity and mortality it is difficult to measure accurately the effect of a DSS on such outcomes.

As it is clear from this chapter, medical informatics researchers can play an important role in supporting the process of guideline development, dissemination, and implementation. 


\section{References}

1 Field M, Lohr K, (Eds). Guidelines for clinical practice: from development to use. Institute of Medicine, Washington, DC: National Academy Press. 1992.

2 Shaneyfelt TM, Mayo-Smith MF, Rothwangl J. Are guidelines following guidelines? The methodological quality of clinical practice guidelines in the peer-reviewed medical literature. JAMA. 1999 May 26;281(20):1900-1905.

3 Clinical Practice Guidelines. [cited 01.05.2009]; Available from:http://www.openclinical.org/ guidelines.html Technol Assess Healthcare. 2003 Winter;19(1):148-157.

13 Shiffman RN, Dixon J, Brandt C, Essaihi A, Hsiao A, Michel G, et al. The GuideLine Implementability Appraisal (GLIA): development of an instrument to identify obstacles to guideline implementation. BMC Med Inform Decis Mak. 2005;5:23.

14 Appraisal of Guidelines for Research and Evaluation. [cited 16.12.2009]; Available from: www. agreecollaboration.org

15 Shiffman RN, Shekelle P, Overhage JM, Slutsky J, Grimshaw J, Deshpande AM. Standardized reporting of clinical practice guidelines: a proposal from the Conference on Guideline Standardization. Ann Intern Med. 2003 Sep 16;139(6):493-498.

16 Shiffman RN, Michel G, Essaihi A, Thornquist E. Bridging the guideline implementation gap: a systematic, document-centered approach to guideline implementation. J Am Med Inform Assoc. 2004 Sep-Oct;11(5):418-426. 
17 Boxwala AA, Tu S, Peleg M, Zeng Q, Ogunyemi O, Greenes RA, et al. Toward a representation format for sharable clinical guidelines. J Biomed Inform. 2001 Jun;34(3):157-169.

18 Grimshaw J, Eccles M, Thomas R, MacLennan G, Ramsay C, Fraser C, et al. Toward evidencebased quality improvement. Evidence (and its limitations) of the effectiveness of guideline dissemination and implementation strategies 1966-1998. J Gen Intern Med. 2006 Feb;21 Suppl 2:S14-20.

19 Prior M, Guerin M, Grimmer-Somers K. The effectiveness of clinical guideline implementation strategies--a synthesis of systematic review findings. J Eval Clin Pract. 2008 Oct;14(5):888-897.

20 Grol R, Zwaard A, Mokkink H, Dalhuijsen J, Casparie A. Dissemination of guidelines: which sources do physicians use in order to be informed? Int J Qual Healthcare. 1998 Apr;10(2):135140.

21 Lin KW, Slawson DC. Identifying and using good practice guidelines. Am Fam Physician. 2009 Jul 1;80(1):67-70.

22 Dufour JC, Fieschi D, Fieschi M. Coupling computer-interpretable guidelines with a drugdatabase through a web-based system--The PRESGUID project. BMC Med Inform Decis Mak. 2004 Mar 2;4:2.

23 Morris $\mathrm{AH}$. Developing and implementing computerised protocols for standardization of clinical decisions. Ann Intern Med. 2000 Mar 7;132(5):373-383.

24 Patel VL, Allen VG, Arocha JF, Shortliffe EH. Representing clinical guidelines in GLIF: individual and collaborative expertise. J Am Med Inform Assoc. 1998 Sep-Oct;5(5):467-483.

25 Gillois P, Chatellier G, Jaulent MC, Colombet I, Fieschi M, Degoulet P. From paper-based to electronic guidelines: application to French guidelines. Medinfo. 2001;10(Pt 1):196-200.

26 Fox J, Black E, Chronakis I, Dunlop R, Patkar V, South M, et al. From guidelines to careflows: modelling and supporting complex clinical processes. Stud Health Technol Inform. 2008;139:4462.

27 Trivedi MH, Kern JK, Marcee A, Grannemann B, Kleiber B, Bettinger T, et al. Development and implementation of computerised clinical guidelines: barriers and solutions. Methods Inf Med. 2002;41(5):435-442.

28 Woolf SH, Grol R, Hutchinson A, Eccles M, Grimshaw J. Clinical guidelines: potential benefits, limitations, and harms of clinical guidelines. BMJ. 1999 Feb 20;318(7182):527-530.

29 Biondich PG, Downs SM, Carroll AE, Shiffman RN, McDonald CJ. Collaboration between the medical informatics community and guideline authors: fostering HIT standard development that matters. AMIA Annu Symp Proc. 2006:36-40.

30 Shekelle PG, Woolf SH, Eccles M, Grimshaw J. Clinical guidelines: developing guidelines. BMJ. 1999 Feb 27;318(7183):593-596.

31 Goud R, Hasman A, Strijbis AM, Peek N. A parallel guideline development and formalization strategy to improve the quality of clinical practice guidelines. Int J Med Inform. 2009 Aug;78(8):513-520. 
32 Peleg M, Gutnik LA, Snow V, Patel VL. Interpreting procedures from descriptive guidelines. J Biomed Inform. 2006 Apr;39(2):184-195.

33 Musen MA, Fagan LM, Combs DM, Shortliffe EH. Use of a domain model to drive an interactive knowledge-editing tool. Int J Man-Mach Stud. 1987 Jan;26(1):105-121.

34 Peleg M, Tu SW. Design patterns for clinical guidelines. Artif Intell Med. 2009 Sep;47(1):1-24.

Tran N, Michel G, Krauthammer M, Shiffman RN. Embedding the Guideline Elements Model in Web Ontology Language. AMIA Annu Symp Proc. 2009 November 14:645-649.

Votruba P, Miksch S, Seyfang A, Kosara R. Tracing the formalization steps of textual guidelines. Stud Health Technol Inform. 2004;101:172-176.

37 Svatek V, Ruzicka M. Step-by-step mark-up of medical guideline documents. Int J Med Inform. $2003 \mathrm{Jul} ; 70(2-3): 329-335$.

38 Shahar Y, Young O, Shalom E, Galperin M, Mayaffit A, Moskovitch R, et al. A framework for a distributed, hybrid, multiple-ontology clinical-guideline library, and automated guidelinesupport tools. J Biomed Inform. 2004 Oct;37(5):325-344. Leslie SJ, Hartswood M, Meurig C, McKee SP, Slack R, Procter R, et al. Clinical decision support software for management of chronic heart failure: development and evaluation. Comput Biol Med. 2006 May;36(5):495-506.

40 Goldstein MK, Hoffman BB, Coleman RW, Tu SW, Shankar RD, O'Connor M, et al. Patient safety in guideline-based decision support for hypertension management: ATHENA DSS. Proc AMIA Symp. 2001:214-218.

41 Vogelzang M, Zijlstra F, Nijsten MW. Design and implementation of GRIP: a computerised glucose control system at a surgical intensive care unit. BMC Med Inform Decis Mak. 2005;5:38.

42 Kawamoto K, Houlihan CA, Balas EA, Lobach DF. Improving clinical practice using clinical decision support systems: a systematic review of trials to identify features critical to success. BMJ. 2005 Apr 2;330(7494):765.

43 Mollon B, Chong J, Jr., Holbrook AM, Sung M, Thabane L, Foster G. Features predicting the success of computerised decision support for prescribing: a systematic review of randomised controlled trials. BMC Med Inform Decis Mak. 2009;9:11.

44 Wright A, Sittig DF. A four-phase model of the evolution of clinical decision support architectures. Int J Med Inform. 2008 Oct;77(10):641-649.

45 Wright A, Sittig DF. A framework and model for evaluating clinical decision support architectures. J Biomed Inform. 2008 Dec;41(6):982-990. Patel VL, Branch T, Wang D, Peleg M, Boxwala A. Analysis of the process of encoding guidelines: a comparison of GLIF2 and GLIF3. Methods Inf Med. 2002;41(2):105-113.

47 Wang D, Peleg M, Tu SW, Boxwala AA, Ogunyemi O, Zeng Q, et al. Design and implementation of the GLIF3 guideline execution engine. J Biomed Inform. 2004 Oct;37(5):305-318. De Clercq P, Kaiser K, Hasman A. Computer-Interpretable Guideline formalisms. Stud Health Technol Inform. 2008;139:22-43. 
49 Isern D, Moreno A. Computer-based execution of clinical guidelines: a review. Int J Med Inform. 2008 Dec;77(12):787-808.

50 Sonnenberg FA, Hagerty CG. Computer-interpretable clinical practice guidelines. Where are we and where are we going ? Yearb Med Inform. 2006:145-158.

51 Peleg M, Tu S, Bury J, Ciccarese P, Fox J, Greenes RA, et al. Comparing computer-interpretable guideline models: a case-study approach. J Am Med Inform Assoc. 2003 Jan-Feb;10(1):52-68.

52 Wang D, Peleg M, Tu SW, Boxwala AA, Greenes RA, Patel VL, et al. Representation primitives, process models and patient data in computer-interpretable clinical practice guidelines: a literature review of guideline representation models. Int J Med Inform. 2002 Dec 18;68(13):59-70.

53 Seroussi B, Bouaud J, Chatellier G. Guideline-based modeling of therapeutic strategies in the special case of chronic diseases. Int J Med Inform. 2005 Mar;74(2-4):89-99.

54 Bouaud J, Seroussi B, Falcoff H, Venot A. Design factors for success or failure of guidelinebased decision support systems: an hypothesis involving case complexity. AMIA Annu Symp Proc. 2006:71-75.

55 de Clercq PA, Blom JA, Korsten HH, Hasman A. Approaches for creating computer-interpretable guidelines that facilitate decision support. Artif Intell Med. 2004 May;31(1):1-27.

56 Wang D, Peleg M, Bu D, Cantor M, Landesberg G, Lunenfeld E, et al. GESDOR - a generic execution model for sharing of computer-interpretable clinical practice guidelines. AMIA Annu Symp Proc. 2003:694-698.

57 Tu SW, Campbell J, Musen MA. The SAGE guideline modeling: motivation and methodology. Stud Health Technol Inform. 2004;101:167-171.

58 Tu SW, Campbell JR, Glasgow J, Nyman MA, McClure R, McClay J, et al. The SAGE Guideline Model: achievements and overview. J Am Med Inform Assoc. 2007 Sep-Oct;14(5):589-598.

59 Fox JP, Bury J, Humber M, Rahmanzadeh A, Thomson R. Publets: clinical judgement on the web. Proc AMIA Symp. 2001:179-183.

60 Kawamoto K, Lobach DF. Proposal for fulfilling strategic objectives of the U.S. Roadmap for national action on clinical decision support through a service-oriented architecture leveraging HL7 services. J Am Med Inform Assoc. 2007 Mar-Apr;14(2):146-155.

61 Kawamoto K, Lobach DF. Design, implementation, use, and preliminary evaluation of SEBASTIAN, a standards-based Web service for clinical decision support. AMIA Annu Symp Proc. 2005:380-384.

62 Dominguez E, Perez B, Zapata M. Towards a Traceable Clinical Guidelines Application. Methods Inf Med. 2009 Dec 21.

63 Skonetzki S, Gausepohl HJ, van der Haak M, Knaebel S, Linderkamp O, Wetter T. HELEN, a modular framework for representing and implementing clinical practice guidelines. Methods Inf Med. 2004;43(4):413-426.

64 Musen MA, Eriksson H, Gennari JH, Tu SW, Puerta AR. PROTEGE-II: a suite of tools for 
development of intelligent systems from reusable components. Proc Annu Symp Comput Appl Med Care. 1994:1065.

65 Johnson PD, Tu SW, Musen MA, Purves I. A virtual medical record for guideline-based decision support. Proc AMIA Symp. 2001:294-298.

66 Marcos M, Roomans $H$, ten Teije A, van Harmelen F. Improving medical protocols through formalization: a case study. In Proc of the 6th Int Conf on Integrated Design and Process Technology (IDPT-02). 2002.

67 Martens JD, Werkhoven MJ, Severens JL, Winkens RA. Effects of a behaviour independent financial incentive on prescribing behaviour of general practitioners. J Eval Clin Pract. 2007 Jun;13(3):369-373.

68 Bindels R, Hasman A, Kester AD, Talmon JL, De Clercq PA, Winkens RA. The efficacy of an automated feedback system for general practitioners. Inform Prim Care. 2003;11(2):69-74.

69 Carton M, Auvert B, Guerini H, Boulard JC, Heautot JF, Landre MF, et al. Assessment of radiological referral practice and effect of computer-based guidelines on radiological requests in two emergency departments. Clin Radiol. 2002 Feb;57(2):123-128.

70 Lugtenberg M, Burgers JS, Westert GP. Effects of evidence-based clinical practice guidelines on quality of care: a systematic review. Qual Saf Healthcare. 2009 Oct;18(5):385-392.

71 Butzlaff M, Vollmar HC, Floer B, Koneczny N, Isfort J, Lange S. Learning with computerised guidelines in general practice?: A randomised controlled trial. Fam Pract. 2004 Apr;21(2):183188.

72 Jousimaa J, Makela M, Kunnamo I, MacLennan G, Grimshaw JM. Primary care guidelines on consultation practices: the effectiveness of computerised versus paper-based versions. A cluster randomised controlled trial among newly qualified primary care physicians. Int J Technol Assess Healthcare. 2002 Summer;18(3):586-596.

73 Shiffman RN, Liaw Y, Brandt CA, Corb GJ. Computer-based guideline implementation systems: a systematic review of functionality and effectiveness. J Am Med Inform Assoc. 1999 MarApr;6(2):104-114.

74 Shiffman RN, Brandt CA, Liaw Y, Corb GJ. A design model for computer-based guideline implementation based on information management services. J Am Med Inform Assoc. 1999 Mar-Apr;6(2):99-103.

75 Hunt DL, Haynes RB, Hanna SE, Smith K. Effects of computer-based clinical decision support systems on physician performance and patient outcomes: a systematic review. JAMA. 1998 Oct 21;280(15):1339-1346.

76 Garg AX, Adhikari NK, McDonald H, Rosas-Arellano MP, Devereaux PJ, Beyene J, et al. Effects of computerised clinical decision support systems on practitioner performance and patient outcomes: a systematic review. JAMA. 2005 Mar 9;293(10):1223-1238.

77 Ambresin AE, D’Acremont V, Mueller Y, Martin O, Burnand B, Genton B. www.fevertravel.ch: an online study prototype to evaluate the safety and feasibility of computerised guidelines 
for fever in returning travellers and migrants. Comput Methods Programs Biomed. 2007 Jan;85(1):19-31.

78 Overhage JM, Tierney WM, Zhou XH, McDonald CJ. A randomised trial of "corollary orders" to prevent errors of omission. J Am Med Inform Assoc. 1997 Sep-Oct;4(5):364-375.

79 Goud R, Engen-Verheul Mv, Keizer Nd, Bal R, Hasman A, Hellemans IM, et al. The effects of computerised decision support on barriers to guideline implementation: A quality study in outpatient cardiac rehabilitation. 2009.

80 Goud R, Jaspers MW, Hasman A, Peek N. Subjective usability of the CARDSS guideline-based decision support system. Stud Health Technol Inform. 2008;136:193-198.

81 Chan AS, Coleman RW, Martins SB, Advani A, Musen MA, Bosworth HB, et al. Evaluating provider adherence in a trial of a guideline-based decision support system for hypertension. Medinfo. 2004;11(Pt 1):125-129.

82 Worrall G, Chaulk P, Freake D. The effects of clinical practice guidelines on patient outcomes in primary care: a systematic review. CMAJ. 1997 Jun 15;156(12):1705-1712.

83 Eagle KA, Montoye CK, Riba AL, DeFranco AC, Parrish R, Skorcz S, et al. Guideline-based standardized care is associated with substantially lower mortality in medicare patients with acute myocardial infarction: the American College of Cardiology's Guidelines Applied in Practice (GAP) Projects in Michigan. J Am Coll Cardiol. 2005 Oct 4;46(7):1242-1248.

84 Heselmans A, Van de Velde S, Donceel P, Aertgeerts B, Ramaekers D. Effectiveness of electronic guideline-based implementation systems in ambulatory care settings - a systematic review. Implement Sci. 2009 Dec 30;4(1):82.

85 Bryan C, Boren S. The use and effectiveness of electronic clinical decision support tools in the ambulatory/primary care setting: a systematic review of the literature. Inform Prim Care. 2008;16(2):79-91(13).

86 Grimshaw JM, Russell IT. Effect of clinical guidelines on medical practice: a systematic review of rigorous evaluations. Lancet. 1993 Nov 27;342(8883):1317-1322.

87 Cabana MD, Rand CS, Powe NR, Wu AW, Wilson MH, Abboud PA, et al. Why don't physicians follow clinical practice guidelines? A framework for improvement. JAMA. 1999 Oct 20;282(15):1458-1465.

88 Lugtenberg M, Zegers-van Schaick JM, Westert GP, Burgers JS. Why don't physicians adhere to guideline recommendations in practice? An analysis of barriers among Dutch general practitioners. Implement Sci. 2009;4:54.

89 Espeland A, Baerheim A. Factors affecting general practitioners' decisions about plain radiography for back pain: implications for classification of guideline barriers--a qualitative study. BMC Health Serv Res. 2003 Mar 24;3(1):8.

90 Tehrani FT, Roum JH. Intelligent decision support systems for mechanical ventilation. Artif Intell Med. 2008 Nov;44(3):171-182.

91 Grol R, Dalhuijsen J, Thomas S, Veld C, Rutten G, Mokkink H. Attributes of clinical guidelines 
that influence use of guidelines in general practice: observational study. BMJ. 1998 Sep 26;317(7162):858-861.

92 LaBresh KA. Quality of acute stroke care improvement framework for the Paul Coverdell National Acute Stroke Registry: facilitating policy and system change at the hospital level. Am J Prev Med. 2006 Dec;31(6 Suppl 2):S246-250.

93 Fieschi M, Dufour JC, Staccini P, Gouvernet J, Bouhaddou O. Medical decision support systems: old dilemmas and new paradigms? Methods Inf Med. 2003;42(3):190-198.

94 Gibbons RJ, Antman EM, Smith SC. Has guideline development gone astray? No. BMJ.340:c343.

95 Grol R. Has guideline development gone astray? Yes. BMJ.340:c306. guidelines. Stud Health Technol Inform. 2008;139:63-80.

99 Schmitt J, Balser M, Reif W. Verification of medical guidelines in KIV. Stud Health Technol Inform. 2008;139:253-262.

100 Groot P, Hommersom A, Lucas P. Adaptation of clinical practice guidelines. Stud Health Technol Inform. 2008;139:121-139.

101 Chesani F, Lamma E, Mello P, Montali M, Storari S, Baldazzi P, et al. Compliance checking of cancer-screening Careflows: an approach based on Computational Logic. Stud Health Technol Inform. 2008;139:183-192.

102 Groot P, Hommersom A, Lucas PJ, Merk RJ, Ten Teije A, van Harmelen F, et al. Using model checking for critiquing based on clinical guidelines. Artif Intell Med. 2008 Sep 26.

103 Quaglini S. Compliance with clinical practice guidelines. Stud Health Technol Inform. 2008;139:160-179. 


\section{Design and Implementation of GASTINE}




\section{Abstract}

\section{Objective}

To develop a framework for clinical practice guidelines that not only allows the representation of best practices, but also facilitates reasoning about acceptable alternatives for those best practices.

\section{Method}

Design of an explicit representation formalism of intentions of guidelines and guideline steps. Implementation of this representation and reasoning mechanisms in GASTON, a tool for representing and executing computerised clinical guidelines.

\section{Results}

The developed formalism is used to represent a heart failure clinical guideline. It is demonstrated that the representation of intentions provides the needed flexibility to avoid unnecessary errors and warnings.

\section{Conclusion}

By using an explicit representation of the intentions of guidelines and guideline steps, a flexible decision support system can be built that does not check only the adherence to the formal guideline but evaluates clinical activities in light of the intention of the guidelines. 


\subsection{Introduction}

Clinical practice guidelines (CPGs) are "systematically developed statements to assist physician and patient decisions about appropriate healthcare for specific circumstances" [1]. Use of clinical guidelines benefits healthcare by improving its quality, lowering medical cost of treatments and reducing practice variability [2]. Woolf et al. [3] point out that patients, physicians, insurance companies and managers define quality of healthcare differently and evidence about the effectiveness of guidelines is incomplete. Further, there are some factors that restrict physicians' adherence to a guideline. Cabana et al. [4] reported seven general categories of barriers to physicians' guideline adherence such as the physician's knowledge (lack of awareness, lack of familiarity), attitudes (lack of agreement, lack of self-efficacy) and behaviour (external barriers, environmental factors). Until now clinical practice guidelines were mainly available in paper form. Physicians are not aware of the majority of existing guidelines and as a consequence their adherence to the guidelines is low. We observe an increase of guidelines implemented in Decision Support Systems (DSSs)[5-7], where the computer helps users to follow the guideline. Automated guidelines may give a better overview of existing and implemented guidelines, however, their developers need to take into account that the new approach may introduce new errors. Koppel et al. [8], discuss a system of computerised physician order entry and point out new kinds of errors the system may introduced. Existing automated guidelines may also be difficult to understand, as became apparent in our initial work.

In DSSs guidelines are usually implemented as a flowchart and every step to be taken is defined in detail; the guideline is very strict and fixed. However, there are situations where patient characteristics or local constraints do not allow following the DSS directions literally. Physicians may follow a different path than the one specified in the guideline, but still work in the 'spirit' of the guideline. Existing guidelines are not able to cope effectively with alternative actions and may generate reminders or warnings in such situations too early. Winikoff et al. [9] present a simple example of a hungry cat (intelligent agent), who has a plan how to reach food on the table. When circumstances change and his plan can not be accomplished, he goes for an alternative set of actions, but still with the purpose to reach his goal, getting food. He needs to change his primary plan to fulfil his intention. Similarly a physician may select an alternative treatment than specified in the guideline, because of a patient specific situation or a local or temporary constraint, not foreseen when the guideline was developed.

The objective of this chapter is to demonstrate a possible technique to overcome some of these problems when implementing guidelines into a DSS. We introduce intentions and show a new way of building guidelines. Development of mechanisms to reason with these intentions will be necessary. 
There are other groups who implemented intention-based guidelines. Shahar et al. [10] present the use of intentions in ASBRU. In this case, intentions are temporal patterns of provider actions or patient states, to be achieved, maintained, or avoided and are inferred from care provider actions or explicitly stated by the provider. This thesis discusses a different approach. We present the use of intentions to check the adequacy of alternative actions taken by the physician and to explain why certain actions have been executed (use of purposes).

\subsection{Definitions}

Before specifying intention Problem Solving Methods (PSMs), we define some terms commonly used in association with intentions [11].

Intention. An intention is a determination to act in a certain way or to do a certain thing; it is a high level goal description which is abstract and not quantified. (e.g., lowering blood pressure)

Goal. A goal is the objective of one's efforts; it is a measurable instance of the intention. Fulfilling the goal means fulfilling the intention. (e.g., blood pressure less than 130/85 within 5 months).

Plan. A plan is a proposed method of getting from one situation to another. A plan contains a series of actions to be carried out.

Action. An action is a process of acting or doing something. We distinguish two kinds of actions: atomic (e.g., do laboratory test) and composite (e.g., titration of medication).

Purpose. A purpose is an expected qualitative outcome of an action. (e.g., the purpose of diuretics is to reduce fluid retention).

\subsection{Implementation}

\subsubsection{First model}

In our work we made use of the heart failure protocol developed by a cardiologist from the Cardiology Department of the Academic Hospital Maastricht. Further, in cooperation with this specialist, we implemented it in the GASTON system [12].

We started our work with the formalization of the paper-based guideline and local protocol.

The guideline is built into the GASTON system as a flowchart by use of components with predefined logic, so called primitives. Figure 3.1 shows a part of this flowchart at the highest level. The top primitives (diamonds) of both charts are eligibility decisions 
which allow entering the flowchart only if predefined criteria as stated in the primitives are fulfilled. All white rectangular elements are plans; they represent other flowcharts containing actions.

While designing the guideline we noticed that flowcharts became very complex and difficult to oversee. Without detailed knowledge about the implemented guideline it is very difficult for others to follow the logic or to find possible mistakes.

We evaluated our model with a cardiologist and we faced another problem; the model lacks flexibility. Even though the model was developed according to the guideline and the local protocol, in some cases physicians would like to bypass some steps. For example, the protocol describes exactly in what dosage steps the drug should be increased while in some cases the physician would like to increase the dose faster. In the latter case, current systems would send a warning to the user, but a system that uses intentions will accept the choice of the physician, as it is in the spirit of the guideline.

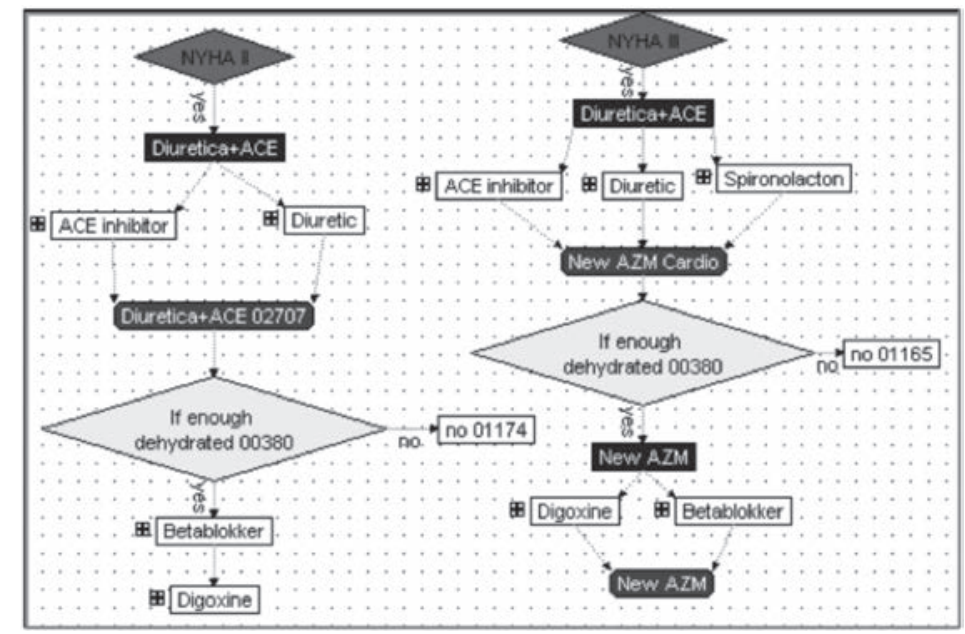

FIGURE 3.1 Part of the heart failure guideline flowchart. The top primitives (diamonds) of both charts are eligibility decisions, dark rectangles "Diuretics +ACE" are branch steps, white rectangular elements are plans (they represent other flowcharts containing actions), "diuretic +ACE 02707" and New AZM Cardio" are synchronization steps.

We also used the runtime version of the heart failure protocol for educational purposes. During the course, third year medical students were confronted with the guideline and the system. The students had to enter patient data in an Electronic Patient Record (EPR) that was interfaced with GASTON. After this data entry the students were asked to find out what the paper guideline proposed for treatment. Afterwards, they could ask the system for advice. The system provided patient specific advice very quickly and in this way they could experience the advantage of having computerised system support. 
Based on the experience we gained we decided to improve our system. The flowchart should be less complex, allowing relatively easy maintenance. Also users should be able to follow the logic of the flowchart and must be able to understand the rationale of the different steps. The system should also be able to accept, at least initially, alternative actions that fulfil the same purpose. We therefore investigated the adequateness of the use of intentions, as already introduced in the ASBRU project. We want to show that using intentions in developing computer-based guidelines can make them more flexible, more insightful and reduce the number of unnecessary warnings.

\subsection{General idea}

The intention is executed by Problem Solving Methods which are domain-independent reasoning components and specify patterns of behaviour which can be reused across applications [13]. We implemented intention PSMs as an addition to the GASTON framework.

The GASTON framework consists of three parts: a guideline editor, with which intention based guidelines are created; a guideline ontology to store medical knowledge; and an execution engine. The physician communicates with the system via an EPR. The EPR has bidirectional communication with the execution engine. During runtime, an execution engine reads data entered into the EPR and uses these data to determine the action that is most appropriate for the current patient (Figure 3.2). The determination of this action is based on the information from the implemented guideline (taking next step) and/or the guideline Terminology Editor (for checking purposes).

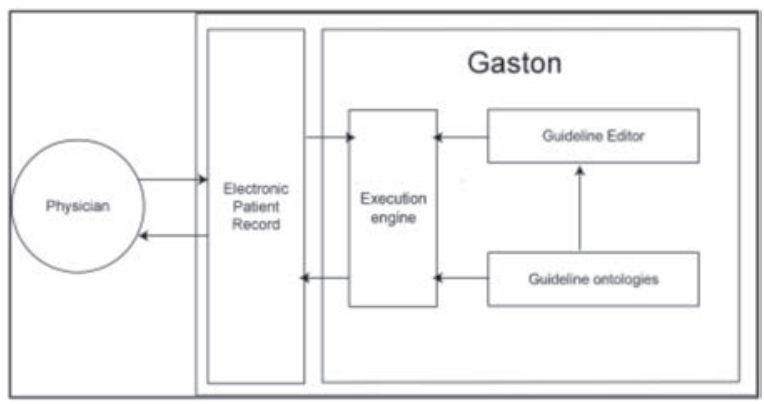

FIGURE 3.2 General architecture of the system.

During the encounter with the patient, the physician enters all information concerning the patient into the EPR. After data entry, our system determines what kind of information has been entered and to which guideline that information relates. It compares the action 
of the physician with the one stored in the guideline. If they match, the system waits for the next entry. If the actions do not match, the system compares the purpose of the action of the physician with the purpose of the action stored in the guideline. If the purposes are the same no warning or error is sent.

\subsection{Guideline design}

A guideline with intentions is built in a number of phases. During the design first a flowchart of intentions has to be described, then every intention individually and finally the plans with actions.

In the first phase, (Figure 3.3), the main intention (number 1 ) of the guideline is specified and decomposed into sub-intentions (numbers 2-5). The intentions can be carried out in parallel (numbers 2-4) or in sequence (numbers 2 and 5). The intention on a higher level is fulfilled only when the set of the intentions on the lower level is satisfied. When the intention cannot be decomposed into other intentions it will refer to a flowchart of actions that aim at fulfilment of the goal of the intention (level of plans). As showed in Figure 3.3, intentions 1 and 4 decompose into other intentions, while 2, 3, 5, 6 and 7 have a plan of actions attached.

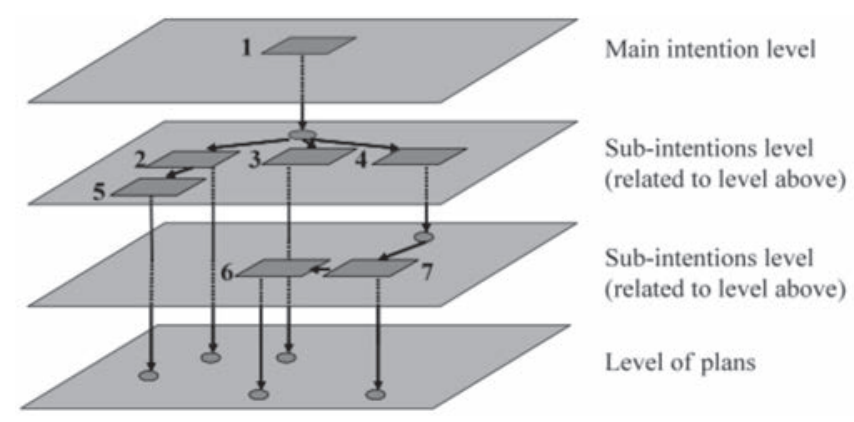

FIGURE 3.3 Multi-level approach to model guidelines with intentions.

In the second phase, each intention is defined via the structure and contents tabs of the GASTON editor (see Figure 3.4). Using the content tab it can be indicated whether the intention is decomposed or not (resulting in the tab sub-intentions or the tab actions respectively). The sub-intentions tab presents the flowchart of the intentions on the lower level (Figure 3.5), while the actions tab shows the reference to the level of plans. 


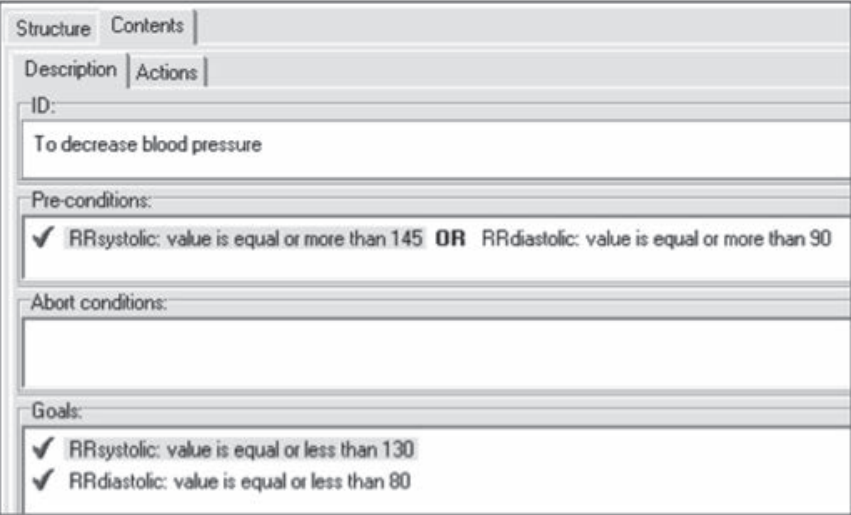

FIGURE 3.4 Description of an intention.

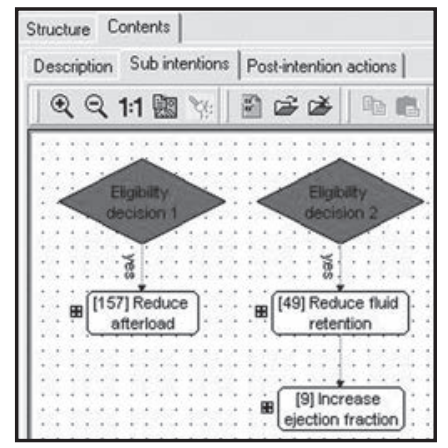

FIGURE 3.5 Flowchart of sub-intentions within intention.

We made en explicit distinction between action and sub-intentions tabs to eliminate the possibility of some designing errors. Firstly, if the action tab is active, the designer knows that the intention PSM (s)he works on refers to plans of actions; if the sub-intentions tab is active, the designer may introduce only other intention PSMs into this intention PSM. This protects from interference between the levels in our multi-level approach. Secondly, in the action tab all available primitives can be used, while in the sub-intention tab just AND/OR connections are possible.

Further, via the contents tab the intention is described. The description contains a number of components: the name of the intention (ID), pre-conditions, abort conditions, and the goal (Figure 3.4).

In the first component (intention ID), we describe the abstract goal, the intention we want to achieve. The name is standardized and described by terms stored in the guideline ontology. In the preconditions part the designer describes all information needed to decide whether this intention can be entered. When the preconditions of an intention 
are satisfied it can be entered. We use abort conditions to describe situations when the execution of this intention has to be aborted. The goal component describes the measurable part of an intention. It indicates in measurable terms which conditions have to be reached for a successful termination of the intention. The goal may be specified using temporal constraints. The goal can be set in two ways: by the physician, based on the patient condition (e.g., for one patient the targeted blood pressure is $140 / 90$, while for another patient it is $120 / 80$ ) or by the guideline designer, based on general medical knowledge (e.g., calcium level has to be between 3.5 and $5.5[\mathrm{mmol} / \mathrm{L}])$.

Actions can be combined into plans (e.g., deciding on a certain drug and determining the optimal dosage). Such a plan can be reused, so storing them in a database enables the use of the same plan in different guidelines.

Both goal and flowcharts use a predefined structure to represent complex temporal criteria enclosed in clinical guidelines. The framework of temporal aspects contains:

a a temporal ontology, which defines time-related concepts (e.g., time points or intervals) and the corresponding temporal operators that relate these concepts and are needed to reason about time (Figure 3.6);

b a temporal data model;

c a mechanism for executing temporal criteria (temporal query mechanism) [14].

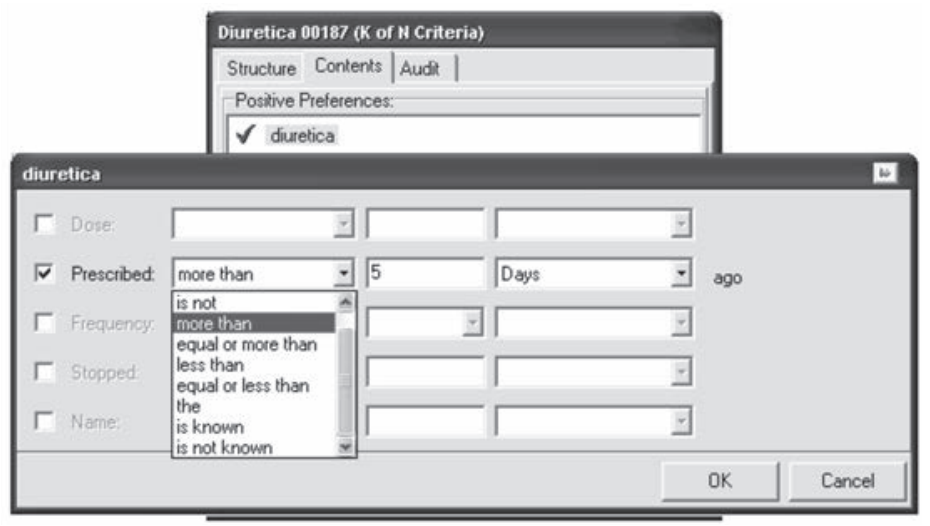

FIGURE 3.6 Representation of temporal operators in GASTON framework. 


\subsection{Terminology editor}

With the Terminology Editor (TE) the ontology of medical acts and treatments such as drugs, laboratory tests and imaging methods is specified. All entries of the knowledge base are grouped into classes (ontology) and have predefined properties. For every class of the data, like drugs, diseases, laboratory tests, we describe mappings to the same concepts in the EPR, their purpose and their attributes (Figure 3.7).

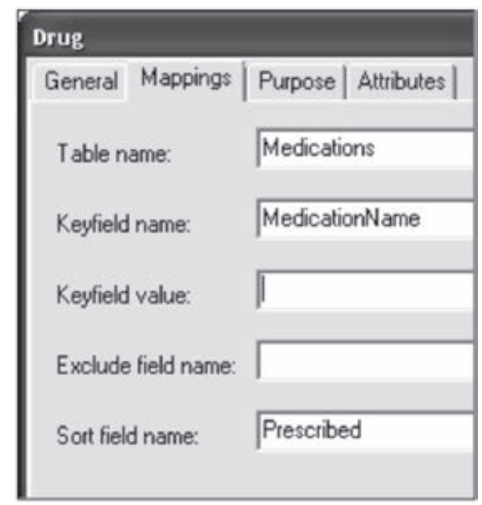

FIGURE 3.7 Properties window of the Mappings of the Drug Class of Terminology Editor.

Mappings. Usually there is a discrepancy between the terminology used in EPR systems and the ones used in the guideline system. Mappings are needed to make the connection. In Figure 3.7 the mappings for the drugs group are shown. A few attributes have to be described before the system knows where the right information in the EPR database can be found (e.g., in the terminology in the Gaston Editor we find an ontology group named Drug, which conforms to the Table name - Medications in the EPR database. Keyfield name - MedicationName tells GASTON in which field of this table it can look for the specific drug names. All medications in the EPR database are sorted by Prescribed, a fact that Gaston finds in the Sort field name).

Attributes. Every class of data has predefined features. Among attributes of the drug class we can distinguish 'prescribed' (followed by time constraints), 'dose', or 'frequency' (Figure 3.6). Attributes are needed to define temporal aspects during the design phase.

Purpose. The purpose of an action is used to match the actions stated by a physician in the EPR with those mentioned in the guideline. During runtime the ontology is used to retrieve the purpose of each action of the physician and to compare this purpose with the purpose of the action mentioned in the guideline. Rather than comparing the actions themselves, we compare their purposes. For example, if the guideline suggests MRI and the physician selects $\mathrm{CT}$, as long as the purposes are the same (imaging of soft tissue for 
example) no alarm should be generated by the system. The purpose of those alternative actions should be available to the system. In order to be able to compare purposes we express them by the use of standardized terminology.

\subsection{Guideline execution engine}

As mentioned above the guideline execution engine uses PSMs to execute the intention descriptions.

The GASTON framework consists of a guideline execution environment that translates defined guidelines into a more efficient representation, which is read in and processed by an execution engine.

A guideline execution engine processes the guideline representation directly. As execution speed is a very important requirement at this stage, the representation language is described in an efficient format to be interpretable by an execution engine in real-time. Finally, the guideline execution engine is able to exchange information with the outside world, such as external EPR systems [12].

To give the correct advice to the user but also to generate reminders or alerts only when needed, we designed a mechanism of pruning non-applicable actions. In cases when certain constraints, previous experience or information stored in the EPR prohibit certain actions, the system will advice the physician to not execute them. For example, if there are a number of alternative treatments mentioned in the guideline and there was a situation in the past that the patient didn't tolerate one of them, the system will note it and take it into account while giving feedback. Or the other way around, if in the past the patient reacted on one of the treatment much better than on the others, the system is not sending any alerts if the physician directly chooses this treatment the next time.

\subsection{Discussion}

In this chapter we concentrate our efforts on extending existing software to obtain computer-based guidelines that are easier to implement and evaluate. This reduces complexity, enhances flexibility in processing received data, and may increase physician's acceptance of the guideline. We introduce intentions as a solution to those problems.

Our main focus was on developing a system that can communicate with the physician in a flexible way (through advice and alerts), allowing him to take a path that may not be completely according to the guideline, but in its spirit. This provides the physician with 
the option to make decisions based on his previous experience or on local agreements. He can also take into account the patient's preferences, which is a very important issue. By studying the use of these alternatives we can obtain insight into the actions preferred by the physicians and determine the reasons of possible deviations from the guideline.

Actions that are not exactly according to the guideline but still in its spirit are stored in the database. After executing a specified number of such actions, the system may remind the physician that he is not executing the preferred action as mentioned in the guideline. A decision support system based on intentions does not only address 'what is the next action' but also 'why is this the next action'. Intentions can thus be used to explain the user why the actions have to be taken. This feature can be exploited to familiarise oneself with the guideline or for educational purposes.

Designing intention based guidelines using flowcharts, where intentions are executed in parallel or in sequence, gives us control about the guideline flow and makes giving relevant advice easier. The work with a heart failure guideline provides us with an example of such a use of intentions. Two kinds of medication are usually given in parallel: an ACE inhibitor and diuretics. A beta-blocker is given to the patient when the intention of the prescription of diuretics (no fluid retention) is fulfilled. The intentions of diuretics and beta-blocker are thus considered in sequence.

Intentions can be decomposed into other intentions (into lower level intentions) or they can refer to the plan of actions. In the first case the intention on a higher level is fulfilled when the proper logical combination of intentions at the lower level is fulfilled. In the second case a goal has to be described for the intention. This can be done in two ways: by the physician based on the current patient state or by the guideline designer obtained from medical knowledge. We can show the use of this feature in a simple example.

The intention slot of the intention description may read for example: "Lowering blood pressure". The goal in this situation might be set by the physician, because the value of the blood pressure that he would like to reach may vary from patient to patient.

Setting the goal by the guideline designer can be done, for example, in the heart failure guideline when the calcium level has to be checked. The normal level of calcium for all patients should be within a fixed range and is based on medical knowledge. Sometimes a goal is not easily expressible. In the case of the ACE inhibitor the strategy is to raise the dose as high as possible without having side effects. When such strategies are available they take the place of a goal.

We envision that implementing intention based guidelines might help evaluating the guideline. If some actions in the guideline are only recommended by a few physicians to their patients, we could conclude that those actions might not be common anymore or 
that users might not be aware of some new possibilities and may need extra information or training.

We have implemented the guideline for heart failure into the new system. The nurse practitioners from the Cardiology Department of the Academic Hospital Maastricht assisted us and validated the model. We extended the terminology editor with facilities for intention based guidelines. 


\section{References}

1 Clinical Practice Guidelines. [cited; Available from: http://www.openclinical.org/guidelines. html

2 Grimshaw JM, Russell IT. Effect of clinical guidelines on medical practice: a systematic review of rigorous evaluations. Lancet. 1993 Nov 27;342(8883):1317-22.

3 Woolf SH, Grol R, Hutchinson A, Eccles M, Grimshaw J. Clinical guidelines: potential benefits, limitations, and harms of clinical guidelines. Bmj. 1999 Feb 20;318(7182):527-30.

4 Cabana MD, Rand CS, Powe NR, Wu AW, Wilson MH, Abboud PA, et al. Why don't physicians follow clinical practice guidelines? A framework for improvement. Jama. 1999 Oct 20;282(15):1458-65.

5 Leslie SJ, Hartswood M, Meurig C, McKee SP, Slack R, Procter R, et al. Clinical decision support software for management of chronic heart failure: development and evaluation. Comput Biol Med. 2006 May;36(5):495-506.

6 Goldstein MK, Hoffman BB, Coleman RW, Tu SW, Shankar RD, O'Connor M, et al. Patient safety in guideline-based decision support for hypertension management: ATHENA DSS. Proc AMIA Symp. 2001:214-8.

7 Vogelzang M, Zijlstra F, Nijsten MW. Design and implementation of GRIP: a computerised glucose control system at a surgical intensive care unit. BMC Med Inform Decis Mak. 2005;5:38.

8 Koppel R, Metlay JP, Cohen A, Abaluck B, Localio AR, Kimmel SE, et al. Role of computerised physician order entry systems in facilitating medication errors. Jama. 2005 Mar 9;293(10):1197203.

9 Winikoff M, Padgham L, Harland J, Thangarajah J. Declarative and Procedural Goals in Intelligent Agent Systems. 2002 Toulouse, France, April 22-25, 2002.

10 Shahar Y. The Asgaard project: a task-specific framework for the application and critiquing of time-oriented clinical guidelines Artificial Intelligence in Medicine. 1998;14(1):29-51(23).

11 [cited; Available from: www.dictionary.com

12 de Clercq PA, Hasman A, Blom JA, Korsten HH. Design and implementation of a framework to support the development of clinical guidelines. Int J Med Inform. 2001 Dec;64(2-3):285-318.

13 Fensel D, Motta E. Structured Development of Problem Solving Methods IEEE Translations on Knowledge and data engineering. 2001;13(6):913-32.

14 Triantafyllou E, Kokkinou E, de Clercq PA, Peek NB, Korsten HHM, Hasman A. Representation and Execution of Temporal Criteria for Guideline-Based Medical Decision Support at the Intensive Care Unit. BNAIC Brussel; 2005; 2005. 


\section{Implementing guidelines in GASTINE}

Published as:

Latoszek-Berendsen AA, de Clercq PA, van den Herik J, and Hasman A. Intention-based expressions in GASTINE. Methods Inf Med. 2009; 48(4):391-6 


\section{Abstract}

\section{Objectives}

(1) to evaluate the design of the framework for computerised intention-based clinical practice guidelines; (2) to implement runtime features such as plan recognition and backtracking.

\section{Method}

To evaluate the design, we implemented the heart failure guideline into GASTINE, a tool for representing and executing intention-based clinical guidelines.

\section{Result}

Description of the current implementation of intention-based expressions in GASTINE and analysis of some generic shortcomings. Explanation of how these shortcomings are addressed. Presentation of how plan recognition and backtracking work and how they improve the system.

\section{Conclusion}

The improved guideline system is rather flexible, i.e., it allows deviations from the guideline as long as they are in the spirit of the guideline. The recognition of actions as intended by the users facilitates a flexible Decision Support System. The intentions are used to explain why certain actions were suggested. Therefore it is assumed that showing the intention behind suggested actions provides a better insight into why these actions are advised. 


\subsection{Introduction}

Future healthcare will benefit from computerised guidelines stored in Decision Support Systems (DSSs). Healthcare providers, insurance companies, and patients all have their requirements that are often in conflict with each other. The insurance companies aim at (1) structured work with standardization of treatments and (2) the possibility of saving costs, while the healthcare providers and patients emphasise (3) the need for more flexibility in making choices, and (4) better patient outcomes [1-3]. Dealing with these conflicting demands is a challenge for the designer of DSSs.

The quality of the guidelines used in such systems is an important issue. There are different research groups that perform advanced work in creating and testing guidelines, both paper and computer based. Some groups study the value of high-quality guidelines [4], they take into account scope and purpose, clarity and presentation, applicability etc. They conclude that guidelines produced within structured and coordinated programs and by governmental agencies have higher scores than guidelines produced in other ways. Other groups focus on how to go from a paper to a computer-based guideline. Patel et al. [5] distinguished the following steps in the translation process: (1) generating the guideline, (2) translating it into GLIF, (3) implementing the GLIF representation in the clinical institution's application system, and (4) interpreting the guideline by a clinician. The group of Gillois [6] presented the electronic Clinical Practice Guidelines (eCPG) "life cycle" of four steps, viz. (1) to structure the text, (2) to model the text by using a representation model, (3) to implement the result, and (4) to evaluate it. The process of creating eCPGs is repeated.

There are also research groups that examined the consequences of the expertise of the development group on the interpretation of the paper guideline [5]. In their work, Patel et al. [5] present results of an experiment where the same guideline was implemented by three different groups of experts. Their results suggest that depending on the level of experience and knowledge, different groups interpret the same clinical guideline in different ways. The work of the above mentioned groups gives us information on advantages and disadvantages of paper-based and computer-based guidelines. It identifies the problems that developers face during the design, implementation and evaluation of the guideline, e.g., too much information can limit the flexibility, while too little information can lead to misinterpretations.

Problems such as lack of flexibility or insufficient guideline clarity might be solved by going one step further in the direction of Intention-based Decision Support Systems [7]. Intention-based DSSs leave more room for the physician. In these systems, there is a possibility for successful cooperation between a physician and the machine, since both have their own strengths and weaknesses. As Shahar points out, a physician has better 
access to some types of patient data, while a machine has a better overview over the guideline specification and may detect more easily complex temporal patterns in the patient data. In the situation where the physician only partly follows the guideline, the intention-based system should be able to determine whether the healthcare provider follows the contents of the guideline at least according to its spirit.

We have addressed the problem of intention-based guidelines and how to implement them in a well-known existing framework for building computerised guidelines - GASTON [8]. In an earlier article we proposed a generic approach for representation and handling of guidelines using an Intention Problem Solving Method (PSM) [9].

In this chapter we describe how intentions are to be implemented and present a new system GASTINE (GASTon INtentional Expressions). We explain our implementation of intentions more extensively. We also describe the incorporated changes in implementation that resulted after a first test period during which we discovered three shortcomings. We present two runtime features, which are important for building flexible and insightful DSSs. Finally, we present a provisional evaluation (first results).

\subsection{Background information}

We start with explaining five terms commonly used in association with intentions [10]. Intention. An intention is a determination to act in a certain way or to do a certain thing; it is a high-level goal description that is abstract and not quantified (e.g., lowering blood pressure).

Goal. A goal is the objective of one's efforts; it is a measurable instance of the intention. Fulfilling the goal means fulfilling the intention (e.g., blood pressure less than 130/85 within 5 months).

Plan. A plan is a proposed method of going from one situation to another. A plan contains a series of actions to be carried out; they are called composite actions (e.g., titration of medication).

Action. An action is a process of acting or doing something (e.g., do laboratory test).

Purpose. A purpose is an expected qualitative outcome of an action (e.g., the purpose of a diuretic is to reduce fluid retention).

The formalism that we use for expressing intentions is characterised by two independent classes of reusable components: (1) domain ontologies and (2) problem-solving methods (PSMs).

Domain ontologies provide a domain of discourse; they model entities and relationships for a particular area of interest. Every class of the ontology has attributes. For the intention-based approach, we introduced one more attribute which is Purpose. It can 
have a list of values, so we can directly determine what the purposes of a certain action are when this action is encountered in the EPR. Purpose is included at the right class level. For all diuretics the same Purpose is entered at that class level and then inherited by all the individual diuretic drugs. The purpose of an action has to match an intention in the guideline. For the values of both Purpose and Intention standardized terms are used. In GASTON, it was sufficient to define in the domain ontology only those domain concepts that appear in the guideline. To be able to reason with alternative actions with the same purpose, one has to include those alternative actions also in the domain ontology.

PSMs represent generic strategies to solve stereotypical tasks, independent of the system's application domain. PSMs are role-limiting by nature, meaning that the PSMs impose specific problem-solving roles on domain knowledge. These problem-solving roles are called knowledge roles, which give an abstract description of the function that the knowledge has to play. When refining a PSM to a certain domain, the knowledge roles are mapped onto domain knowledge [11]. GASTON uses a task chaining method. As soon as an intention is satisfied control is passed to the next task. This mechanism was also used in GASTINE.

\subsection{Problems encountered after the implementation of the heart failure guideline}

We implemented the Dutch national heart failure guideline into the first version of GASTINE. We used this quite extensive guideline as a starting point. In cooperation with a nurse practitioner we formulated (and extracted) intentions underlying the guideline. We used the titration protocols for medications designed at the cardiology department at the Academic Hospital Maastricht, the Netherlands. We specified (1) pre-conditions, (2) abort conditions, (3) goals, and (4) plans (strategies to be used) to reach the goals and to fulfil the intentions.

\subsubsection{The oedema problem}

The design of our original intention PSM was based on the work by Winikoff [12]. He claimed that as soon as a goal is reached the intention is fulfilled and pending actions can be dropped. As described in section 4.2, in our system an intention is satisfied as soon as the goal is reached. For scheduling actions (and intentions), we used a task chaining approach. Subsequent actions or intentions are started after the preceding task has ended. In medicine we meet many situations where an action, e.g., giving a medication, should be continued even when the goal of giving this medication is reached. Let us provide an example. 
For the treatment of oedema, the goal is satisfied when the oedema has disappeared. As mentioned above the rule was that when the goal is reached, the intention is "fulfilled" and then is dropped. In this case, however, when oedema has disappeared, the physician is supposed to reduce the dosage of the diuretic to the lowest level still consistent with oedema absent. Even when the goal is reached, there are therefore still actions that have to be carried out before the intention is completely satisfied.

\subsubsection{The reduce heart rate problem}

In the heart failure guideline we again face the above-mentioned problem but now related to the task-chaining scheduling procedure that is used in GASTON. In this case, other actions or sub-intentions should be started as soon as the goal of the previous intention is reached. The 'reduce heart rate' intention should be executed after the 'eliminate fluid retention' intention. It has to start as soon as the goal (that oedema has disappeared) is satisfied and not when all the actions have been carried out that belong to the previous intention.

As mentioned in the previous subsection, the system was changed in such a way that the intention 'eliminate fluid retention' is only satisfied when the dosage of the medication given to the patient is decreased to the lowest level for which oedema is still absent. So, the intention 'reduce heart rate' will start too late. The task is to avoid this problem. The problems discussed in 4.3.1 and 4.3.2 are related. They have been solved by modifying the Intention PSM as explained in 4.4 .

\subsubsection{Different actions, the same purpose}

A third problem we faced after implementation was making it possible to accept different alternative composite actions having the same purpose. Our line of reasoning is as follows: as soon as a physician starts an action that has the same purpose as the action suggested by the guideline we accept the alternative action and leave the control to the physician. Our system would thus suppress part of the guideline and continue at the location in the guideline where the same action is advised as a following action advised by the physician in the EPR.

The approach worked well with atomic alternative actions but dealing with composite actions (e.g., alternative medications with different titration schemes) gave new problems. This problem was solved as follows. When an alternative composite action with the same purpose is recognised by the system, the system will replace the part of the guideline concerning the originally suggested composite action by the set of actions belonging to the alternative. No sign of recognition or warning will be given. Only when the physician perseveres in deviating from the guideline when treating more patients in the same way he will be given a reminder. 


\subsection{Final structure of the PSM}

During the design of the guideline, we need to specify which domain knowledge fulfils each of the knowledge roles needed by the PSM. The Intention PSM determines the flow through a specific part of the guideline and also plays a similar function as eligibility criteria. An Intention PSM is modelled as a flowchart of decisions steps. The information needed by an intention PSM is requested via a template, as presented in Figure 4.1. The template contains the problem solving roles, necessary for the PSM to function. For an Intention PSM these roles are pre-conditions, abort conditions (only used if all plans would fail) and goals. Similarly the plans (actions and post-intention actions) can be entered into the template (using other tabs). After entering these data, the intention PSM is fully instantiated. Since the PSM consists of a number of decision steps, it can be executed in the same way as the remaining part of the guideline.

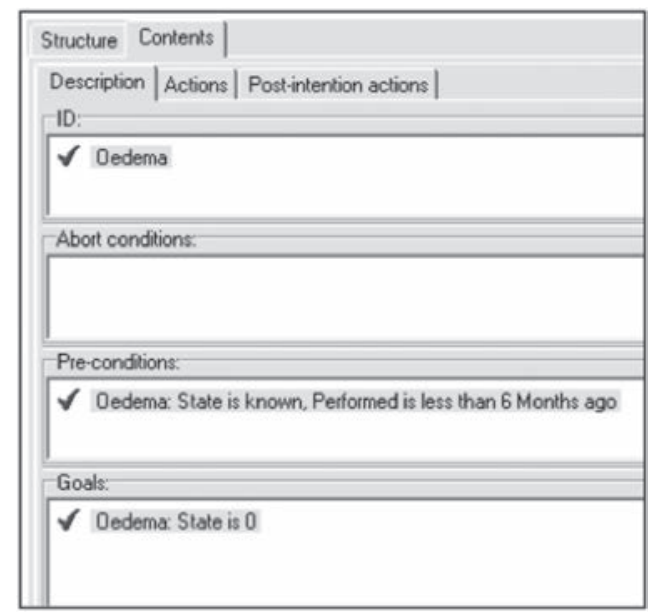

FIGURE 4.1 Information for the Intention Problem Solving Method.

In Figure 4.2 the working of the PSM is further explained. An intention is activated when its abort conditions are false and pre-conditions are true. The goal plays a role of decision step. If the goal is false (not yet reached), a sub-intention or action plan should follow. If the goal is true, the plan stored in the post-intention actions should be executed.

If the pre-conditions are false no actions are taken and the intention is not activated. In case of oedema, the pre-condition is oedema: State is known. Performed less than 6 Months ago. This means that an examination of the patient was performed less than 6 months ago and oedema was found. 


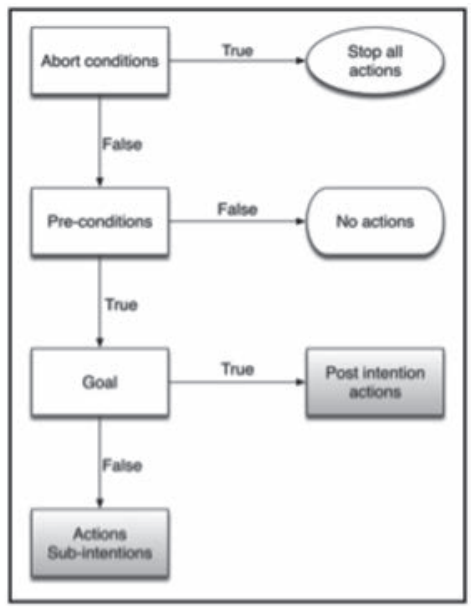

FIGURE 4.2 Flow diagram of Intention Problem Solving Method.

The problem noted in subsection 4.3.1 is solved by the addition of post intention actions. When the goal of an intention is satisfied, actions that still must be performed are specified as post-intention actions (e.g., maintaining or tempering doses), see Figure 4.2. The problem noted in subsection 4.3.2 was solved by the addition of pre-conditions. In GASTINE we now have both a task chaining procedure and a control flow based on pre-conditions. With the possibility of pre-conditions the reduce heart rate task will be started as soon as the goal of the 'eliminate fluid retention' task is reached while in the mean time the 'eliminate fluid retention' task will still be active in reducing the dosage of the diuretic via the post intention actions.

Information regarding Intention PSMs and actions is entered with the help of the Terminology Editor (TE) of GASTINE. TE is defined in the Protégé framework: a methodology and a set of tools to develop knowledge based systems [13]. With Protégé, knowledge engineers are able to define concepts and relations to create domain ontologies as well as method ontologies. In GASTINE, representation elements are arranged in such ontologies.

\subsection{Runtime features}

In this section we present two main features introduced to our system, which are important for building flexible, and insightful decision support systems. First, we discuss re-activating intentions and actions (4.5.1) and plan recognition (4.5.2). Then we continue with backward tracking (4.5.3) and complete the section by a validation of the system (4.5.4). 


\subsubsection{Re-activating intentions and actions}

GASTINE is implemented as a non-memory system. So during each communication with the EPR system, it is determined which intentions are relevant and which are not.

For example, when the 'eliminate fluid retention' sub-intention is encountered, it is important for GASTINE to know if there is any information about oedema in the patient medical history. GASTINE searches for this information in the EPR and if it is mentioned that oedema was present, it concludes that this sub-intention is relevant (the precondition is met).

Then the part of the flowchart behind the relevant intentions is traversed with the help of the patient data. Again if less current data are needed GASTINE will request them from the EPR system. After it has become clear that the pre-condition has been satisfied, it is checked whether the goal has been reached (see Figure 4.2).

If that is the case (oedema is absent) we enter the post intention actions (see Figure 4.2). The system checks whether the oedema value was the current value and if so it suggests either maintaining or decreasing the dosage.

If the value is not current then it is checked whether the patient is on diuretics. If not then the oedema problem was apparently solved earlier and nothing has to be done. If diuretics were given, then the current value of oedema should be asked and then one should act accordingly.

If the goal is not satisfied and the last oedema observation was not current, the system will ask for the current value and then act on it. If the value was current and no diuretics were described then it is advised to prescribe diuretics. If the patient was already on diuretics treatment, the dose should be maintained or increased.

GASTINE provides pro-active as well as re-active feedback. The communication between the EPR and GASTINE can be both ways. In our implementation the initiative lies with the EPR user.

In order to obtain pro-active feedback, the user has to press an Advice button on the screen of the EPR system. When the user does that, a trigger is sent to GASTINE. GASTINE then asks the EPR system for the most current data and uses these data for navigating through the flowchart.

Re-active feedback is given when the user indicates that he is ready and wants to logout. Again, GASTINE is triggered, asks for new data from the EPR, and uses them to check whether the physician's actions were according to the guideline prescriptions.

\subsubsection{Plan recognition}

As stated above, we extended GASTON with a mechanism to make the system more flexible. The system should be able (1) to reason with intentions and (2) to check the purposes of performed actions. 
To realise these research ideas, we started to look at plan recognition techniques that are able to predict the user's intentions based on his actions. Shahar et al. [14] presented such a plan recognition technique. The authors suggest that an analysis using five different levels should enable one to abstract the motives and plans of a physician and compare them with the plans of the guideline. In their work, Sips et al. [15] presented how in Asbru they used intentions to represent the underlying physician's actions and used the intentions as the basis for matching performed actions to prescribed actions.

However, we faced considerable difficulties in abstracting information from actions made by users. The problem was among others that not all actions are relevant for a certain intention. Moreover, the process in itself also turned out to be quite time consuming. We decided to develop a method, which is less universal but is certainly adequate for the goals set. The key point is that our system is designed to "follow" a physician's intentions instead of recognizing them. More general intention-based systems would process data about the actions of the physician to recognize the plan of the physician, which then is compared with the plan in the guideline. We straightforwardly compare a single data entry in the Electronic Patient Record (EPR) with the "action to be performed according to the intention" formulated in the guideline. More precisely, our system first compares the name of the action entered into the EPR with the name of the action, which should be performed according to the guideline.

If there is a match, the user works according to the guideline. If there is no match, the system retrieves from the domain ontology all actions having the same purpose as the purpose of the action of the guideline. If one of those actions matches the physician's action, this action is accepted. As was said before, for composite actions like medications with a titration scheme, part of the guideline is replaced by the plan containing the modified titration scheme of the alternative medication, see 4.3.3.

\subsubsection{Backward tracking}

The possibility of backtracking the performed actions and their corresponding intentions is especially important for educational purposes. When someone would like to know why a certain action was recommended or carried out, he can ask for an explanation (by clicking the dark "i" button) (see Figure 4.3). Further in Figure 4.4 we present the backtracking information generated by GASTINE after the user asked for more detailed information, here for example about the advice concerning diuretic treatment.

The system first displays the intention, then the sub-intentions, and only thereafter decision steps to explain the path that led to the current action. Our conjecture is that such an answer to the question 'Why was the action A advised?' will be better understandable, than when only the actions that were carried out before action A (if any) are presented. When, in our example from Figure 4.3 and Figure 4.4, the user asks why 


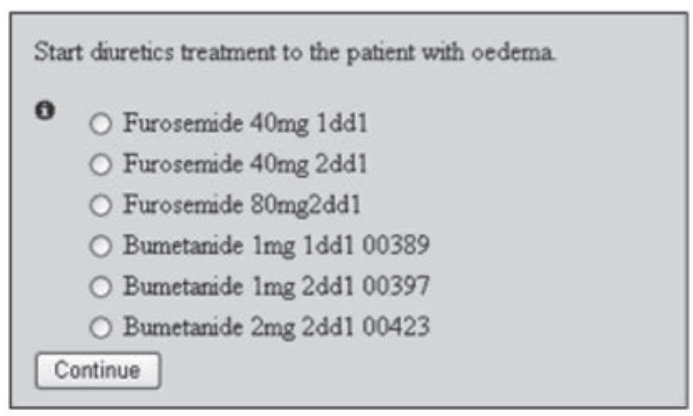

FIGURE 4.3 Possibility of backtracking by asking for more information via the "i" button.

a diuretic treatment should be started the system mentions that it wants to treat heart failure and that one of the tasks is to treat oedema when it is present. So the system may mention that oedema occurred in the patient for the first time and no diuretics have been prescribed until now. Therefore the action should be to prescribe diuretics (Furosemide or Bumetanide). As presented in Figure 4.3, there is a possibility of generating flexible advice to the user by giving him a list of possible actions to choose from.

In Figure 4.4 after each "More" it is explained what type of reasoning provided the actual results. In this case we get two decision steps that say that diuretics were described neither more than one day ago nor today. This just means that until now no diuretics were prescribed. The reason that these two steps could not be collapsed into one is that another action follows when "The diuretics is prescribed more than one day ago" is True. However, a conclusion of a decision step can be based on the result of e.g., ANDing a number of rules. These rules together with their evaluation are then shown in the next step.

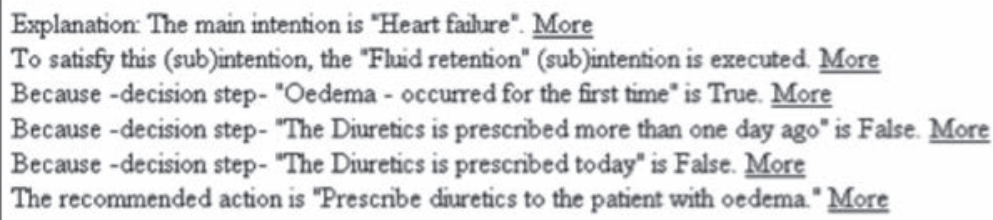

FIGURE 4.4 Backtracking information.

\subsubsection{Validation of the system}

To validate the system we entered into the EPR eight different patient states with corresponding history describing patients in different stages of treatment. By using the backtracking mechanism we could check whether the relevant part of the guideline was correctly executed. The patient states were selected in such a way that all features such as task chaining, the use of pre-conditions, and post intention actions were covered. We may conclude that the system worked well and that the earlier problems were solved. 


\subsection{Discussion}

In this chapter we presented GASTINE, a software package that supports the building and execution of intention based clinical practice guidelines.

As stated before, our system is memory-less meaning that during each communication with the EPR system, it is time and again determined which parts of the guideline are relevant and which are not. Similar to the scenario approach of Johnson et al. [16], our system provides the possibility to deviate from the guideline recommendation, allows to ask for more information ("i"-button) and generates suggestions based on present data. However, the system developed by Johnson et al. stores the position in the guideline where to start during the next visit. Our system does not remember the previous state of the patient but is generating advice based on the current state and if necessary information from the EPR.

In their work Marcos et al. [17] discuss the critiquing mechanisms in Asbru. They conclude that it is difficult to match expert actions with steps in the protocol. Sips et al. [15] used low-level intentions (context independent intentions) to characterise both actions recorded in the EPR and actions in the guideline. They could match $70 \%$ of the EPR action sequences with guideline execution sequences. We also believe that using the Purposes (the equivalent of low-level intentions) of every action is a crucial element of plan recognition and we implemented it in our system. It is important to realise that the same action can have more than one purpose. It is not clear whether Sips et al. assign more than one low-level intention to an action. The authors also do not mention problems that may arise when a medication with the same purpose is prescribed that has another titration scheme and therefore an other action sequence. In our case, GASTINE uses the information stored in guidelines to follow the physician. The system compares the Purpose of the action mentioned in the guideline and the Purpose of the corresponding action recorded in the EPR. When the Purposes match and the alternative action is a composite action the relevant part of the guideline is replaced by the plan belonging to the alternative action. When the Purposes do not match, the system generates feedback that the user is not working according to the guideline. This approach makes it possible to process intentions of the physician without abstracting them from multiple EPR entries. The introduced backtracking is another interesting feature of GASTINE. When receiving advice, the user can ask "Why?" and receive an answer in the form of (1) an automatically generated path starting from the relevant intention and leading to the current position in the guideline, (2) the relevant part of the paper-guideline, or (3) related published articles. This feature is especially interesting for educational purposes.

Shahar [18] mentions that for patients with AIDS there is a policy that haemoglobin ( $\mathrm{Hb})$ values should be kept above $7 \mathrm{mg} / \mathrm{dl}$ and that an episode of severe anaemia should not 
last for more than 2 weeks. The protocol says that in such a situation the dose of the given drug should be reduced because it lowers $\mathrm{Hb}$ as a side effect. However, the physician prescribed a blood transfusion to the patient and therefore did not follow the guideline. In our approach, the purpose of an action is related to its main effect and not to possible side effects. However, this situation can still be handled. There is no problem in entering a composite action: (reducing the dose of the drug if the drug is already given) into the system as an action with the purpose to lower the $\mathrm{Hb}$ value. In our case, this medication would have two purposes: as a medication for AIDS and as an $\mathrm{Hb}$ lowering medication (when the dosage is reduced). The system will also recognize that the purpose of blood transfusion is in the spirit of the guideline and will allow the physician to perform this action.

We designed a flexible feedback system, which allows the user to take a path that may not be completely according to the guideline, but in the spirit of the guideline. This provides the practitioner with the option to make decisions based on previous experience or on the local situation. He can also take into account the patient's preferences, which is a very important issue. Of course, these alternative actions have to be entered into the system via the Terminology Editor before they can be considered by the system.

\subsection{Conclusions}

In this article we have shown for some cases that by representing clinical practice guidelines in computerised form and by adding the underlying intentions the resulting computer-interpretable guidelines will be easier to understand and less difficult to specify. The guideline representation that we discussed here supports the (1) design of intentionbased guidelines, (2) their execution, (3) reasoning about intentions during runtime, and (4) providing flexible feedback.

Intention based Decision Support Systems can be beneficial for healthcare in many ways. We believe that they can improve a physician's adherence to the guidelines because the guidelines support and do not disturb the workflow. They make adaptation of the guidelines to the local standards easier and they can make the evaluation of the guideline simpler and faster. A second advantage is that the system implements the original guideline. All local modifications to the guideline are stored separately as alternative actions. 


\section{References}

1 Woolf SH, Grol R, Hutchinson A, Eccles M, Grimshaw J. Clinical guidelines: potential benefits, limitations, and harms of clinical guidelines. BMJ. 1999 Feb 20;318(7182):527-530.

2 Martens JD, Werkhoven MJ, Severens JL, Winkens RA. Effects of a behaviour independent financial incentive on prescribing behaviour of general practitioners. J Eval Clin Pract. 2007 Jun;13(3):369-373.

3 Cabana MD, Rand CS, Powe NR, Wu AW, Wilson MH, Abboud PA, et al. Why don't physicians follow clinical practice guidelines? A framework for improvement. JAMA. 1999 Oct 20;282(15):1458-1465.

4 Burgers JS, Cluzeau FA, Hanna SE, Hunt C, Grol R. Characteristics of high-quality guidelines: evaluation of 86 clinical guidelines developed in ten European countries and Canada. Int J Technol Assess Healthcare. 2003 Winter;19(1):148-157.

5 Patel VL, Allen VG, Arocha JF, Shortliffe EH. Representing clinical guidelines in GLIF: individual and collaborative expertise. J Am Med Inform Assoc. 1998 Sep-Oct;5(5):467-483.

6 Gillois P, Chatellier G, Jaulent MC, Colombet I, Fieschi M, Degoulet P. From paper-based to electronic guidelines: application to French guidelines. Medinfo. 2001;10(Pt 1):196-200.

7 Shahar Y. Automated Support to Clinical Guidelines and Care Plans: The Intention-Oriented View. 2002 [cited; Available from: http://openclinical.org/docs/int/briefingpapers/shahar.pdf de Clercq PA, Hasman A, Blom JA, Korsten HH. Design and implementation of a framework to support the development of clinical guidelines. Int J Med Inform. 2001 Dec;64(2-3):285-318. Latoszek-Berendsen A, Talmon J, de Clercq P, Hasman A. With good intentions. Int J Med Inform. 2007;76(3):440-446.

10 Dictionary. 27/02/2008 [cited; Available from: www.dictionary.com]

11 de Clercq PA, Hasman A, Blom JA, Korsten HH. The application of ontologies and problemsolving methods for the development of shareable guidelines. Artif Intell Med. 2001 Apr;22(1):1-22.

12 Winikoff M, Padgham L, Harland J, Thangarajah J. Declarative and Procedural Goals in Intelligent Agent Systems. 2002 Toulouse, France, April 22-25, 2002.

13 Musen MA, Gennari JH, Eriksson H, Tu SW, Puerta AR. PROTEGE-II: computer support for development of intelligent systems from libraries of components. Medinfo. 1995;8 Pt 1:766-770.

14 Shahar Y, Miksch S, Johnson P. A Task-Specific Ontology for the Application and Critiquing of Time-Oriented Clinical Guidelines. Lecture Notes In Computer Science Proceedings of the 6th Conference on Artificial Intelligence in Medicine in Europe. 1997;1211:51-61.

15 Sips RJ, Braun L, Roos N. Enabling protocol-based medical critiquing. Stud Health Technol Inform. 2006;124:471-476.

16 Johnson PD, Tu S, Booth N, Sugden B, Purves IN. Using scenarios in chronic disease management guidelines for primary care. Proc AMIA Symp. 2000:389-393. 
17 Marcos M, Berger G, van Harmelen F, ten Teije A, Roomans H, Miksch S. Using critiquing for improving medical protocols: harder than it seems. In Proc of the 8th European Conference on Artificial Intelligence in Medicine (AIME-2001). 2001:431-441.

18 Shahar Y, Musen M. Plan recognition and revision in support of guideline-based care. Working notes of the AAAl Spring Symposium on Representing Mental States and Mechanisms. 1995:118-126. 


\section{GASTINE: Verification of the system and implemented heart failure guideline}

GASTINE: Intention-based DSS in practice; results of a validation test. Latoszek-Berendsen A, Janssen-Boyne J, Gorgels A, van den Herik J and Hasman A. 


\begin{abstract}
Objective

Prior to releasing an information system to users, it needs to be well tested. In this chapter we want to answer the question whether we built the GASTINE system right. We verified whether our system is well-engineered and error-free.
\end{abstract}

\title{
Methods
}

We formalised and then implemented the Dutch national heart failure guideline into our system. In this system guidelines were structured on the basis of intentions. For the purpose of verification and validation we designed a straightforward Electronic Patient Record (EPR) with a database created in Office Access. For verification of our system we used the Multiple Condition Coverage (MCC) method. This method requires that all value combinations in the conditions of a decision step are tested. Because the code of the primitives was used in many applications it was not necessary to test all value combinations.

\section{Results}

We found three kinds of errors during verification: (1) Errors made in the criteria logic during the creation of the flowchart via the editor; (2) Errors due to incompleteness of the protocol; (3) A programming problem that occurred when changing the flowcharts. Conclusions: Structuring the guideline according to the different intentions programmed into an object oriented system in combination with the use of the MCC method made an exhaustive verification of the system feasible. For the full test of the system we had to use 2414 value combinations. 


\subsection{Introduction}

Before releasing an information system to users, it needs to be well tested. This is done by system verification and validation.

In 2010 Sergent [1] started his article by repeating the still current definition of verification and validation as stated by Schlesinger in 1979. Schlesinger defined model verification as "ensuring that the computer program of the computerised model and its implementation are correct" and model validation as "substantiation that a computerised model within its domain of applicability possesses a satisfactory range of accuracy consistent with the intended application of the model".

Preece [2] formulated those definitions in the form of supporting-questions that one need to bear in mind while testing the model.

Verification: Are we building the system right?

Validation: Are we building the right system?

In other words, verification is concerned with the question whether a system is wellengineered and error-free. Of course, it will help to determine whether the software is of high quality, but it will not ensure that the system is useful. During model verification, an information system is tested under controlled conditions. Validation is concerned with the question whether the system will meet the customer's actual needs, thus checking if it is useful. During model validation an information system is tested by the use of sets of real patient data.

In this chapter we describe how we verified the GASTINE Decision Support System (DSS). When entering a formalised guideline into the system, via a graphical editor, errors in specifying decision steps, etc. can be made. There could be three kinds of them: (1) with respect to the specification of the conditions, (2) the logical operators between them, and (3) with respect to the links connecting each possible outcome of a decision with the next step. To test whether errors were made during entry of the formalised guideline we tested for each condition those values of the corresponding variable that would lead to another outcome of the condition. Therefore, for each decision step quite a number of value combinations of the different variables involved have to be taken into account. We made use of the Multiple Condition Coverage (MCC) method. This method requires that all value combinations in the conditions of a decision step are tested. In our case we do not need to test all value combinations, because the system primitives like the decision step have already been tested in many applications. Only the conditions and the links to the next steps have to be checked. We therefore do not need to test whether the outcome of the system primitive equals the outcome of the corresponding row in the corresponding truth table. This reduces the number of combinations to be tested considerably. 


\subsection{Formalization}

To investigate whether the new features of GASTINE make a difference in building and executing computerised guidelines as we envisaged, we first formalised and implemented the Dutch national heart failure guideline into our system [3].The formalised guideline was translated into a presentation language similar to GLIF3 but with more features and then implemented into GASTINE. A main feature of the GASTINE system is the use of intentions. In cooperation with a cardiologist and a heart failure nurse practitioner (A.G. and J.B.), we formulated (and extracted) the intentions underlying the guideline's actions. We also implemented the medication titration protocols designed at the cardiology department of the Academic Hospital Maastricht, the Netherlands for providing advice with respect to the dose of the medication. We specified (1) pre-conditions, (2) abort conditions, (3) goals, (4) plans (strategies to be used to reach the goals and to fulfil the intentions), and (5) post-intention actions (strategies that need to be carried out even when the goal of the guideline is reached, e.g., maintenance). We used the implemented heart failure guideline to verify GASTINE.

\subsubsection{The creation of a computerised guideline in GASTINE}

The guideline, translated into a formal language, is represented in GASTINE in the form of a flowchart (or more precisely a task network) [4]. As already explained in Chapter 3, the guidelines entered via the GASTINE editor are created using intentions and primitives. Intentions are implemented via Problem Solving Methods which are domain-independent reasoning components [5]. Primitives are building blocks with predefined logic. There are many primitives available in GASTINE, here we present those that were most used in our project.

Decision step - One of the most important primitives, denoting a situation in the guideline where a decision has to be made. This primitive decides which path to take by executing a number of conditions. Depending on the output of these conditions (yes or no), one of two possible pathways will be followed.

Case step - Is an extension to the Decision Step which, just like the Decision Step, contains a number of conditions (expressed as Boolean logical expressions) that are evaluated. The only difference is that the case step has more outputs, while the Decision Step only offers two. Based on the output of the evaluation of the logical expressions, one of several available alternative paths is executed by the system.

Choice step - This primitive is similar to the Case Step, except that the Choice Step lets the user instead of the system decide.

Start activity (action step) - Via this primitive, GASTINE automatically writes the conclusions, as stored in the condition part of this primitive, into the database so that 
they can be used in subsequent parts of the process described by the guideline.

Recommendation - Primitive that communicates with the users by generating textual information via the user interface or by sending email/SMS messages.

\subsubsection{Pro-active versus re-active mode}

The guideline is implemented both in a pro- and re-active way. This means that either the system can support the physician when making treatment decisions or it can critique the actions of the physician when these actions are not according to the guideline. Those two different approaches require a different mind set during formalisation and implementation of the guideline into GASTINE.

While building pro-active guidelines the question "what should be done next", based on the data that are available, has to be answered, while when building re-active guidelines the question "which actions are suggested by the physician and are they according to or in the spirit of the guideline" needs to be answered.

In the pro-active mode the guideline is executed mostly in three steps.

1 Investigate the state of the patient to check whether this guideline is appropriate,

2 Decide that a certain action is required,

3 Recommend that action.

In the re-active mode the guideline is executed in a slightly different way.

1 Check whether any action is documented by the physician today,

2 Investigate the state of the patient to check whether the guideline under consideration is appropriate,

3 Determine within the re-active part of the flowchart whether the physician's action is according to the guideline,

4 If that is the case, the user works according to the guideline,

5 If there is no match, the system retrieves from the domain ontology all actions having the same purpose as the purpose of the action suggested by the guideline. If one of those actions matches the physician's action, this action is accepted. If there is no match with any possible alternative, a warning will be generated.

Guidelines built in the pro-active mode mainly use decision steps, case steps, choice steps, action steps, and recommendations. Guidelines built in the re-active mode mainly use decision steps, case steps, and recommendations. This difference is due to the fact that in the pro-active mode there is more dialogue with the physician. It is obvious that in the pro-active mode the system has to provide advice in all cases whereas in the re-active mode the system only warns when the physician deviates from the guideline's recommendation. Also, in the pro-active mode there are situations that a guideline cannot continue without further input from the physician. That happens in case of, e.g., the choice step where the physician is consulted about the next steps in 
the treatment. For example, when according to the guideline the patient should receive ACE inhibitor treatment, the physician at that point gets the possibility to choose which ACE inhibitor he would like to prescribe. In addition he will be supported by the system concerning the titration of the medication he chooses. The list of possible medications is not arbitrary; it is based on the local protocols. In the re-active mode the system uses the medication that the physician just prescribed and therefore does not need to use the choice step.

GASTINE is triggered by the physician clicking the "Advice" (more about it in subsection 5.3.4) button or when the physician is closing the patient record in the Electronic Patient Record (EPR). Those actions do not determine which mode has to be started. In a perfect world we would expect that the physician clicks "Advice" when he uses the system in pro-active mode, in case he does not know how to proceed and did not take any actions himself. However, the "Advice" button can be clicked at any moment of the patientphysician encounter to confirm the actions taken by physician. In this case the re-active mode of GASTINE should be triggered and not the pro-active mode. The same is true for "closing the patient record" that we associate with the re-active mode. The record might be closed although the physician did not take any actions. GASTINE should then proceed in the pro-active mode instead of the re-active mode.

How does our system determine whether to proceed in the pro- or re-active mode? This is determined in one of the first steps of every sub-intention. GASTINE checks whether the user has already taken actions or whether only historical data is available. The patient's EPR data are the input values for GASTINE.

Figure 5.1 for example shows the flowchart that leads to a decision to decrease the diuretics medication. Before the system came to this part of the guideline it already checked if diuretics were used by this patient and what the values of relevant parameters such as oedema, creatinine, etc. were. Thus, at the starting point of the flowchart presented in Figure 5.1, it is known that the patient was diagnosed with oedema and received diuretics treatment. The part on the "Yes" side of the first diamond, with the text "Are diuretics prescribed today?", leads to the re-active mode. The part that follows "No" leads to the pro-active mode. However, if the medication was stopped because the previous dose was the lowest dose possible for that medication and the dose can only be decreased by stopping the medication (and therefore no medication would have been prescribed today) the answer to the above question also would be "No" and the system would proceed to the pro-active mode. Therefore one more step is executed: the decision step "Is the lowest dose stopped (today)?" to see if the physician follows the guideline. The data of the EPR are inspected to check that the medication was stopped today and the previous dose was the lowest dose available for that medication. If that is indeed the case the system goes to the "End" primitive and leaves this part of the 
guideline as the guideline was properly followed by the physician. If not, the system proceeds in the pro-active mode where the physician will receive the advice either to lower or to maintain the dose of the diuretic followed by a choice step enabling him to inform the system about his choice. Depending on his choice the 'decrease dose diuretic' or 'maintain dose diuretic' part of the flowchart will be followed. The conditions of both decisions steps "Are diuretics prescribed today?" and "Is the lowest dose stopped (today)?" could have been stored in one primitive. We did not do that to have a better overview of the guideline's steps.

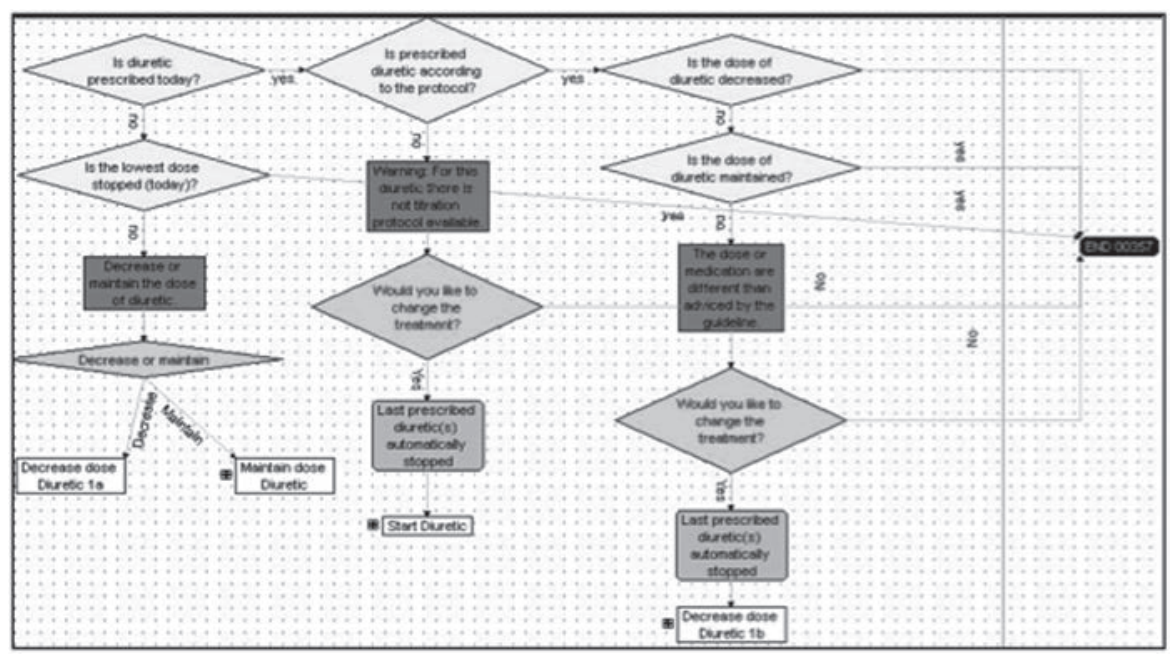

FIGURE 5.1 A sub-intention guideline where in the first steps the system decides about the pro- or re-active mode.

Let us go back to the first diamond. If a medication is prescribed today (the system proceeds in the re-active mode) then the system checks if the prescribed medication is a diuretic by checking the medications ontology and if a titration protocol for this medication is available. If the physician prescribed a known diuretic for which no titration information was implemented in GASTINE the system will follow the arrow "No" from this diamond and warn the physician about the lack of a titration protocol. A physician may still receive general advice based on the patient data (e.g., decrease, increase, or maintain the dosage), but not an exact dose. Also the system provides the possibility to revert to the treatment according to the protocol. If the physician decides to follow the system's advice, the medication he prescribed will be stopped and GASTINE will run further in pro-active mode. The system stays in pro-active mode until all advices within the current intention are given and then the system moves to the next intention. Here again it checks whether the physician acted according to the guideline in the way described above. 
If a diuretic is prescribed today (the system proceeds in the re-active mode) and it is also the one expected by the system, the next step to be checked in the re-active mode is whether the dose prescribed is lower than the last prescribed dose. That is done in the third diamond from the left in the first row. If "Yes", the system leaves this part of the flowchart and moves on. Is the answer "No", then it will check if maybe the dose is maintained. If the dose is maintained, the flowchart can be ended, if not the physician receives the possibility to change his dosage.

Although the pro-active mode does not need to use intentions, the whole guideline is structured with the help of intentions. In this way guideline actions and alternative actions of the same intention are combined in the same part of the flowchart. This makes the interpretation of the guideline easier. In the representation of the guideline without the use of intentions various actions with different goals are intermingled, making it more difficult to follow the logic of the guideline.

\subsubsection{Interpretation problems}

Interpretation problems can occur during formalization of the guideline, for example caused by the presence of vague terms. Terms such as "lower the dose", "in case of high doses, execute action A", and "if value is in the normal range, execute action B" make it impossible to translate the guideline into a formal language without additional information from the expert's group. Also "lower the dose" is ambiguous. It is essential to know what "lower the dose" means in the context of the guideline. The same is true for "in case of high doses". We need to explain to the computer what is understood by the term of "high doses". The role of experts in building the right system right is very important and influences the results of the verification process. Wrong expert interpretations of the guideline will not be detected during the verification process.

\subsection{Verification}

Sailors et al. [6] describe the importance of a good verification of decision support systems. The authors point out how complex an exhaustive test of a DSS is that contains only 10 different Boolean variables (including both input and output variables). In this case 1024 different possible combination of inputs and outputs have to be tested. In our case, when the intentions behind actions are included in the formalised CIG, the complexity of the guideline's flowchart is reduced because the guideline is split up into a number of independent flowcharts and not all variables occur in each flowchart. This results in much fewer combinations to be tested, fewer than would be the case in a network without these branches. In each flowchart the variables presented in the 
conditions control the flow through it, finally leading to a decision to carry out a certain action. The therapy is generally provided over time thus by the next patient-physician encounter GASTINE generates advice based on at that moment available data. GASTINE does not store the location of the last treatment; it is a memory-less system. In each new encounter the system will look at the current set of patient data and will start at the root of the guideline.

\subsubsection{The variables}

In the heart failure guideline we used various variables such as laboratory tests, physical examination data, and medications. In Figure 5.2 we present the three laboratory tests used in the guideline and their attributes. Next to the numeric or textual values, we use also the value "unknown" which means that the value of the attribute cannot be found in the EPR (e.g., there are no results of creatinine tests stored in the EPR). In case of an unknown value the system interprets the condition as "False", which would be "No" in Figure 5.1. In case of, e.g., creatinine, an "unknown" value cannot be treated as "too high" or "too low" as this would lead to the wrong treatment advice. In such circumstances we use a Case Step with three branches where branch 1 relates to actions for a "too high value of creatinine", branch 2 leads to actions for a "too low value of creatinine", and branch 3 leads to actions that need to be taken in case of an "unknown value of creatinine".

\begin{tabular}{|c|c|c|c|c|c|}
\hline \multirow{6}{*}{ 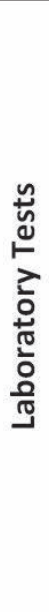 } & & \multirow{2}{*}{ Variable } & \multicolumn{3}{|c|}{ Attributes } \\
\hline & & & \multicolumn{2}{|c|}{ Possible values } & Performed \\
\hline & \multirow{2}{*}{1} & \multirow{2}{*}{ Creatinine } & woman & $\begin{array}{c}\text { unknown } \\
\mathrm{C}<90 \\
\mathrm{C}=90 \\
\mathrm{C}>90\end{array}$ & \multirow{4}{*}{$\begin{array}{c}\text { unknown } \\
\text { less than } 7 \text { days ago } \\
\text { more than } 7 \text { days ago }\end{array}$} \\
\hline & & & man & $\begin{array}{c}\text { unknown } \\
\mathrm{C}<120 \\
\mathrm{C}=120 \\
\mathrm{C}>120\end{array}$ & \\
\hline & 2 & Potassium & & $\begin{array}{l}\text { own } \\
.5 \\
<5.5 \\
.5 \\
.5\end{array}$ & \\
\hline & 3 & NT-proBNP & & $\begin{array}{l}\text { own } \\
N P<125 \\
N P=125 \\
N P>125\end{array}$ & \\
\hline
\end{tabular}

FIGURE 5.2 Laboratory variables and their attributes. 
The group of physical examination data includes 7 variables and their attributes, see Figure 5.3.

\begin{tabular}{|c|c|c|c|c|}
\hline \multirow{9}{*}{ 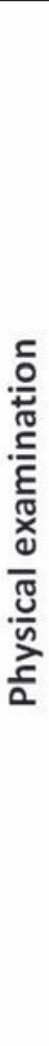 } & & \multirow[b]{2}{*}{ Variable } & \multicolumn{2}{|c|}{ Attributes } \\
\hline & & & Possible values & Performed \\
\hline & 1 & Systolic blood pressure & $\begin{array}{c}\text { unknown } \\
S B D<100 \\
S B D=100 \\
100<S B D<135 \\
S B D=135 \\
S B D>135\end{array}$ & \multirow{7}{*}{$\begin{array}{c}\text { unknown } \\
\text { less than } 1 \text { days ago } \\
\text { more than } 1 \text { day ago }\end{array}$} \\
\hline & 2 & Diastolic blood pressure & $\begin{array}{c}\text { unknown } \\
\mathrm{DBD}<60 \\
\mathrm{DBD}=60 \\
60>\mathrm{DBD}>85 \\
\mathrm{DBD}=85 \\
\mathrm{DBD}>80\end{array}$ & \\
\hline & 3 & Heart rate & $\begin{array}{c}\text { unknown } \\
H R<70 \\
H R=70 \\
H R>70\end{array}$ & \\
\hline & 4 & Weight & $\begin{array}{l}\text { unknown } \\
<80 \mathrm{~kg} \\
=80 \mathrm{~kg} \\
>80 \mathrm{~kg}\end{array}$ & \\
\hline & 5 & NYHA class & $\begin{array}{c}\text { unknown } \\
1,2,3,4\end{array}$ & \\
\hline & 6 & CVP & $\begin{array}{c}\text { unknown } \\
0,1,2\end{array}$ & \\
\hline & 7 & Oedema & $\begin{array}{c}\text { unknown } \\
0,1,2\end{array}$ & \\
\hline
\end{tabular}

FIGURE 5.3 Variables of physical examination and their attributes.

The 7 medication groups contain in total 14 different medications and their attributes as presented in Figure 5. 4. 


\begin{tabular}{|c|c|c|c|c|c|c|c|}
\hline \multirow{16}{*}{$\frac{\frac{5}{8}}{\frac{8}{8}}$} & \multicolumn{3}{|c|}{ Variable } & \multicolumn{4}{|c|}{ Artrifutes } \\
\hline & & Medication rroup & Medications & - different doses & I Frequencies & Prescriotion eate & Stog cate \\
\hline & & & Captopeil & 5 & 1 & \multirow{14}{*}{$\begin{array}{l}\text { unknown } \\
\text { more than } 1 \text { day ago } \\
\text { less than } 1 \text { day ago }\end{array}$} & \multirow{14}{*}{$\begin{array}{l}\text { unknown } \\
\text { more than } 1 \text { day ago } \\
\text { less than } 1 \text { day ago }\end{array}$} \\
\hline & 1 & ACE inhibitor & Enalapeit & 3 & 2 & & \\
\hline & & & Quinapril & 3 & 2 & & \\
\hline & 2 & A H Anranaigr & Valsartan & 2 & 1 & & \\
\hline & 2 & 2. & Losartan & 2 & 1 & & \\
\hline & \multirow{2}{*}{3} & \multirow{2}{*}{ Beta blocker } & Carvedilol & 2 & 3 & & \\
\hline & & & Metoprolol & 1 & 2 & & \\
\hline & \multirow{4}{*}{4} & \multirow{4}{*}{ Diuretics } & Furosemide & 2 & 2 & & \\
\hline & & & Bumetanide & 5 & 4 & & \\
\hline & & & Triomtereen & 1 & 1 & & \\
\hline & & & Spironolecton & 3 & 2 & & \\
\hline & 5 & Diecoxine & Lanoxin & 5 & 2 & & \\
\hline & 6 & Nirtaten & isosorbide & 2 & 2 & & \\
\hline & 7 & Mineral supplement & Slow-K & 2 & 2 & & \\
\hline
\end{tabular}

FIGURE 5.4 Variables of medications and their attributes.

\subsubsection{The role of Intentions}

By using intentions the number of combinations will be reduced to a minimum because the guideline becomes much more structured. With intentions the guideline can be divided into seven flows of which six can be tested separately. The exception concerns the ACEinhibitors and Angiotensin-II-receptor-antagonists. Both flows include actions fulfilling the same intention, but because the medications are from different groups, we could validate them first separately and then together. Not all variables are relevant for each flow. By taking that into account the number of combinations to be checked is reduced significantly. The flow corresponding to the (1) "Reduce afterload" intention concerns medications from the group of ACE inhibitors and Angiotensin-II receptor antagonists; laboratory test: creatinine; physical examination: systolic and diastolic blood pressure, NYHAclass and oedema; (2) the "Fluid retention" intention concerns medications like diuretics and spironolacton; laboratory tests: creatinine, and NT-proBNP; physical examination: NYHAclass, and oedema; (3) the "Increase ejection fraction" intention supports Betablocker prescription; laboratory test: NT-proBNP; physical examination: heart rate, weight, NYHAclass, CVP and oedema; (4) the "Kidney function" intention monitors kidney parameters such as the potassium value; (5) the "Increase contractility" intention is concerned with the titration of digoxin medication; (6) the "Preload reduction" intention is concerned with carrying out the titration of nitrate.

\subsubsection{Multiple Conditions Coverage}

For verification of our system we used the Multiple Condition Coverage (MCC) method. With MCC all necessary combinations of conditions (variable followed by a comparator operator (equal to, larger than, etc.) followed by a threshold value) in the decision step 
are tested. To test whether AND statements were entered correctly we start by assigning every condition the value True (by assigning relevant values to the variables). The outcome of the decision step should then be True. This however does not guarantee that the conditions have been entered correctly. Thresholds used in the conditions may still be wrong. Therefore every variable at least once has to be assigned a value such that the corresponding condition becomes False. When all other conditions are assigned the value True the outcome of the decision should become False. If not, the condition made false was not correctly entered. In OR statements the statement with all conditions false has to be tested. In addition we have to assign every condition at least once the value True while all other conditions are False.

We assume, given the experiences with GASTON in many applications that the relational operators as shown in Figure 5.5 in all primitives function accurately. Therefore we only have to check whether every condition has been entered correctly and whether the outputs (true and false) of the decisions are connected to the correct recommendations. As also visible in this figure, statements like "equal or less than" and "equal or more than " are joined in one operator. In consequence, it also reduces the amount of test values. E.g., the creatinine value from Figure 5.2 will require two and not three values to be checked. When the creatinine value need to be $>=90\left[\mathrm{ml} / \mathrm{min} / 1.73 \mathrm{~m}^{2}\right]$, it is sufficient in our case to take the values $89\left[\mathrm{ml} / \mathrm{min} / 1.73 \mathrm{~m}^{2}\right]$ and $90\left[\mathrm{ml} / \mathrm{min} / 1.73 \mathrm{~m}^{2}\right]$ to test. If the creatinine value is supposed to be $=<90$, we would have to use $90\left[\mathrm{ml} / \mathrm{min} / 1.73 \mathrm{~m}^{2}\right]$ and $91\left[\mathrm{ml} / \mathrm{min} / 1.73 \mathrm{~m}^{2}\right]$.

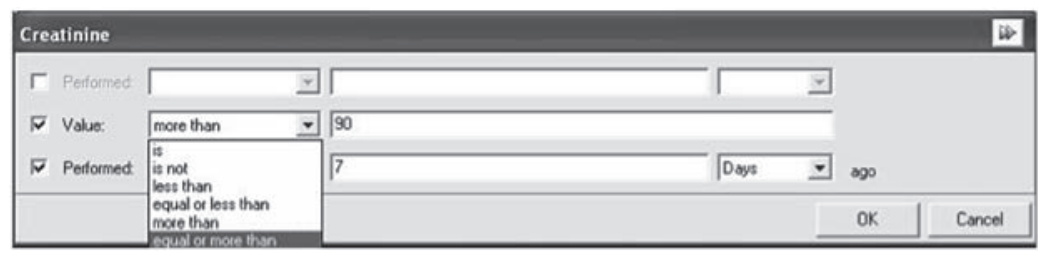

FIGURE 5.5 Relational operators used to describe conditions.

The necessary test cases required for full multiple condition coverage of a decision are given by the truth tables of the logical operators used in the decision. But because we do not have to check the correctness of the decision step primitive not all entries of the table are necessary.

The test value of each variable is entered in the EPR, see subsection 5.3.4. If the condition in GASTINE is wrongly specified, the value stored in the EPR may lead to another truth value for the corresponding condition than expected on the basis of the correct condition. In this way erroneously entered conditions can be detected.

We explain this method by three examples. 


\subsubsection{Example 1. Simple AND conditions.}

Decision Step: "Does patient receive the ACE inhibitor treatment?"

Conditions: ACE inhibitor prescribed less than 6 months ago AND there is no ACE inhibitor StopDate.

Table 5.1 Simple AND conditions.

\begin{tabular}{ccccc}
\hline & ACE $<6 \mathrm{~m}$ & AND & ACE no StopDate & OUTPUT \\
\hline 1 & $\mathrm{~T}$ & $\mathrm{~T}$ & $\mathrm{~T}$ \\
\hline 2 & $\mathrm{~T}$ & $\mathrm{~F}$ & $\mathrm{~F}$ \\
\hline 3 & $\mathrm{~F}$ & $\mathrm{~T}$ & $\mathrm{~F}$ \\
\hline
\end{tabular}

Of the four $\left(2^{2}\right)$ possible combinations only three need to be tested. First it is tested that combination 1 has as output the value True. The output should be False for the second and third combination. In that case it is not necessary to test the combination where both conditions are False. If combination 2 gives as output False then the second condition has been correctly specified. If condition 3 gives as output False then the first condition was specified correctly.

\subsubsection{Example 2. Simple OR conditions.}

Decision Step: Is the prescribed diuretic one of the medications from the protocol? Conditions: Bumetanide prescribed $<1 \mathrm{~d}$ ago OR Furosemide prescribed $<1 \mathrm{~d}$ ago OR Spironolactone prescribed $<1 \mathrm{~d}$ ago.

Table 5.2 Simple OR conditions

\begin{tabular}{|c|c|c|c|c|c|c|}
\hline & Bumetanide & OR & Furosemide & OR & Spironolactone & OUTPUT \\
\hline 1 & $\mathrm{~F}$ & & $\mathrm{~F}$ & & $\mathrm{~F}$ & $\mathrm{~F}$ \\
\hline 2 & $\mathrm{~T}$ & & $\mathrm{~F}$ & & $F$ & $\mathrm{~T}$ \\
\hline 3 & $\mathrm{~F}$ & & $\mathrm{~T}$ & & $\mathrm{~F}$ & $\mathrm{~T}$ \\
\hline 4 & $\mathrm{~F}$ & & $\mathrm{~F}$ & & $\mathrm{~T}$ & $\mathrm{~T}$ \\
\hline
\end{tabular}

Of a total of $8\left(2^{3}\right)$ combinations 4 have to be tested. Of the OR combinations first the one for which all conditions are False is tested. The output should be False. Then with each of the other statements one of the conditions can be tested by making that condition True. The output should then become True. The remaining 4 combinations do not have to be tested. 
5.3.3.3 Example 3. One of the most complicated AND/OR conditions from our guideline. Decision Step: "Does the patient have side effects caused by the last dose of the ACE inhibitor?"

Conditions: (Gender is Female AND CreatinineLast >90 AND CreatinineLast is higher than CreatininePrevious AND CreatinineLast Performed less than 7 days ago) OR (Gender is Male AND CreatinineLast $>120$ AND CreatinineLast is higher than CreatininePrevious AND CreatinineLast Performed less than 7 days ago) OR (RR syst <100 AND Performed less than 1 day ago) OR (RR dias < 60 AND Performed less than 1 day ago) OR (Oedema: State is 2 AND Performed less than 1 day ago) To determine the number of combinations that need to be tested in Step 1 the number of necessary combinations within AND statements is determined and in Step 2 the number of combinations within OR statements.

\section{Step 1}

A: (Gender is Female AND CreatinineLast >90 AND CreatinineLast is higher than CreatininePrevious AND CreatinineLast Performed less than 7 days ago) OR (Gender is Male AND CreatinineLast >120 AND CreatinineLast is higher than CreatininePrevious AND CreatinineLast Performed less than 7 days ago)

Of a total of $36\left(2^{4}+2^{4}+2^{2}\right)$ combinations 8 have to be tested. The number of necessary conditions is grossly reduced due to three reasons. (1) In this statement Gender is an important attribute and takes values Female and Male. In case Gender is Female only the first part of the OR statement has to be tested (the truth values of the other variables in the second part of the OR statement do not matter then). In case Gender is Male, the other part should be tested. (2) Considering the first part of the statement related to Female, 4 combinations need to be tested like an AND statement. In case Gender is Male, the other 4 combinations have to be tested. (3) The whole statement is an OR, thus only three combinations out of four need to be tested (True left side, True right side, and both False). As can be seen in the table, all combinations are included within those 8 cases.

\begin{tabular}{|c|c|c|c|c|c|c|c|c|c|c|c|c|c|c|c|c|c|}
\hline & & Female & AND & $\begin{array}{c}\text { Creat } \\
>90\end{array}$ & AND & $\mathrm{CL}>\mathrm{CP}$ & AND & $\begin{array}{c}\text { Perf } \\
<7 \\
\text { days }\end{array}$ & OR & Male & AND & $\begin{array}{l}\text { Creat } \\
>120\end{array}$ & AND & $\mathrm{CL}>\mathrm{CP}$ & AND & $\begin{array}{c}\text { Perf } \\
<7 \\
\text { days }\end{array}$ & $\begin{array}{l}\text { OUT- } \\
\text { PUT }\end{array}$ \\
\hline \multirow{8}{*}{ A } & 1 & $\mathrm{~T}$ & & $\mathrm{~F}$ & & $\mathrm{~T}$ & & $T$ & & $\mathrm{~F}$ & & - & & - & & - & $\mathrm{F}$ \\
\hline & 2 & $T$ & & $T$ & & $\mathrm{~F}$ & & $T$ & & $\mathrm{~F}$ & & - & & - & & - & $\mathrm{F}$ \\
\hline & 3 & $T$ & & $T$ & & $T$ & & $\mathrm{~F}$ & & $\mathrm{~F}$ & & - & & - & & - & $\mathrm{F}$ \\
\hline & 4 & $T$ & & $T$ & & $T$ & & $T$ & & $\mathrm{~F}$ & & - & & - & & - & $T$ \\
\hline & 5 & $\mathrm{~F}$ & & - & & - & & - & & $T$ & & $\mathrm{~F}$ & & $T$ & & $T$ & $\mathrm{~F}$ \\
\hline & 6 & $\mathrm{~F}$ & & - & & - & & - & & $T$ & & $T$ & & $\mathrm{~F}$ & & $T$ & $\mathrm{~F}$ \\
\hline & 7 & $\mathrm{~F}$ & & - & & - & & - & & $T$ & & $T$ & & $T$ & & $\mathrm{~F}$ & $\mathrm{~F}$ \\
\hline & 8 & $\mathrm{~F}$ & & - & & - & & - & & $T$ & & $T$ & & $T$ & & $T$ & $T$ \\
\hline
\end{tabular}


B: (RR syst <100 AND Performed less than 1 day ago)

\begin{tabular}{ccccc}
\hline & $\begin{array}{c}\text { RR } \\
\text { sys }<100\end{array}$ & AND & $\begin{array}{c}\text { Perf } \\
<1 \mathrm{~d}\end{array}$ & OUTPUT \\
\cline { 2 - 5 } $\mathrm{B}$ & $\mathrm{T}$ & $\mathrm{F}$ & $\mathrm{F}$ \\
\hline & 1 & $\mathrm{~T}$ & $\mathrm{~T}$ & $\mathrm{~T}$ \\
\hline $\mathrm{nyyyy}$ & $\mathrm{F}$ & $\mathrm{T}$ & $\mathrm{F}$ \\
\hline
\end{tabular}

C: (RR DIAST <60 AND Performed less than 1 day ago)

\begin{tabular}{cccccc}
\hline & \multicolumn{1}{c}{$\begin{array}{c}\text { RR } \\
\text { dias }<60\end{array}$} & AND & $\begin{array}{c}\text { Perf } \\
<1 \mathrm{~d}\end{array}$ & OUTPUT \\
\cline { 2 - 5 } $\mathrm{C}$ & $\mathrm{T}$ & $\mathrm{F}$ & $\mathrm{F}$ \\
\hline & 1 & $\mathrm{~T}$ & $\mathrm{~T}$ & $\mathrm{~T}$ \\
\hline & $\mathrm{T}$ & $\mathrm{T}$ & $\mathrm{T}$ & $\mathrm{F}$ \\
\hline 3 & $\mathrm{~F}$ & &
\end{tabular}

D: (Oedema: State is 2 AND Performed less than 1 day ago)

\begin{tabular}{ccccc}
\hline & $\begin{array}{c}\text { Oedema } \\
=2\end{array}$ & AND & $\begin{array}{c}\text { Perf } \\
<1 \mathrm{~d}\end{array}$ & OUTPUT \\
\cline { 2 - 5 } $\mathrm{D}$ & $\mathrm{T}$ & $\mathrm{F}$ & $\mathrm{F}$ \\
\hline 2 & $\mathrm{~T}$ & $\mathrm{~T}$ & $\mathrm{~T}$ \\
\hline 3 & $\mathrm{~F}$ & $\mathrm{~T}$ & $\mathrm{~F}$ \\
\hline
\end{tabular}

Step 2

\begin{tabular}{|c|c|c|c|c|c|c|c|c|}
\hline & A (8) & OR & B (3) & OR & $C(3)$ & OR & $D(3)$ & OUTPUT \\
\hline 1 & $F$ & & $\mathrm{~F}$ & & $\mathrm{~F}$ & & $\mathrm{~F}$ & $\mathrm{~F}$ \\
\hline 2 & $T$ & & $\mathrm{~F}$ & & $\mathrm{~F}$ & & $\mathrm{~F}$ & $T$ \\
\hline 3 & $\mathrm{~F}$ & & $T$ & & $\mathrm{~F}$ & & $\mathrm{~F}$ & $T$ \\
\hline 4 & $\mathrm{~F}$ & & $\mathrm{~F}$ & & $T$ & & $\mathrm{~F}$ & $T$ \\
\hline 5 & $\mathrm{~F}$ & & $\mathrm{~F}$ & & $\mathrm{~F}$ & & $T$ & $T$ \\
\hline
\end{tabular}

In this case 22 test combinations need to be tested here.

With this method we tested if the conditions at every node of our flowchart corresponded to the expected output actions described in the guideline, our gold standard. We applied this method to all flowcharts and tested 2414 combinations before concluding that we built the system right. 


\subsubsection{The Electronic Patient Record}

For the purpose of the verification and validation tests we designed a straightforward EPR with a database created in Office Access. All variable names have to be selected so that there is no issue of wrongly spelled variables. Where possible, the range values were also specified to avoid major mistakes (e.g., weight $0[\mathrm{~kg}]-600[\mathrm{~kg}]$, length $40[\mathrm{~cm}]-250$ [cm], etc.)

In this EPR we could create patients, fill in symptoms, laboratory test values, physical examination values and prescribe and stop medications. The "Advice" button is located in the right top corner of the screen, see Figure 5.6.

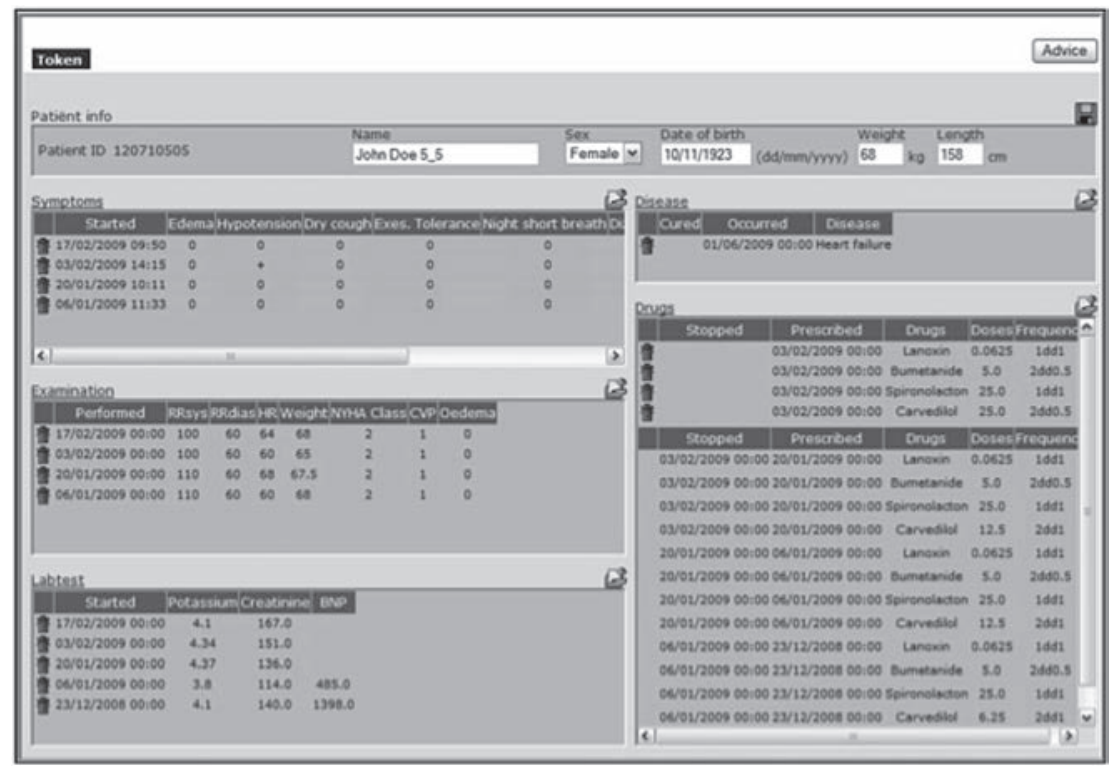

FIGURE 5.6 EPR designed for the needs of this project

The advices and warnings are presented in the window below the EPR screen, see Figure 5.7. There are two ways to see the path that GASTINE takes through the guideline: (1) by inspecting the Evaluation Steps table of the database where the IDs of the guideline, primitives, and conditions are stored in the order they were executed, (2) by turning on a special feature within the user interface of the EPR called "Show steps" that will present below the EPR screen all IDs of the guideline, primitives, and conditions that the system passes. It uses the same window that is used for generating advices, warnings, and recommendations. With this information we can always reproduce the path that GASTINE took to generate advice. The backtracking information is also based on this evaluation information. It is presented in a more compact and comprehensible form, however. 


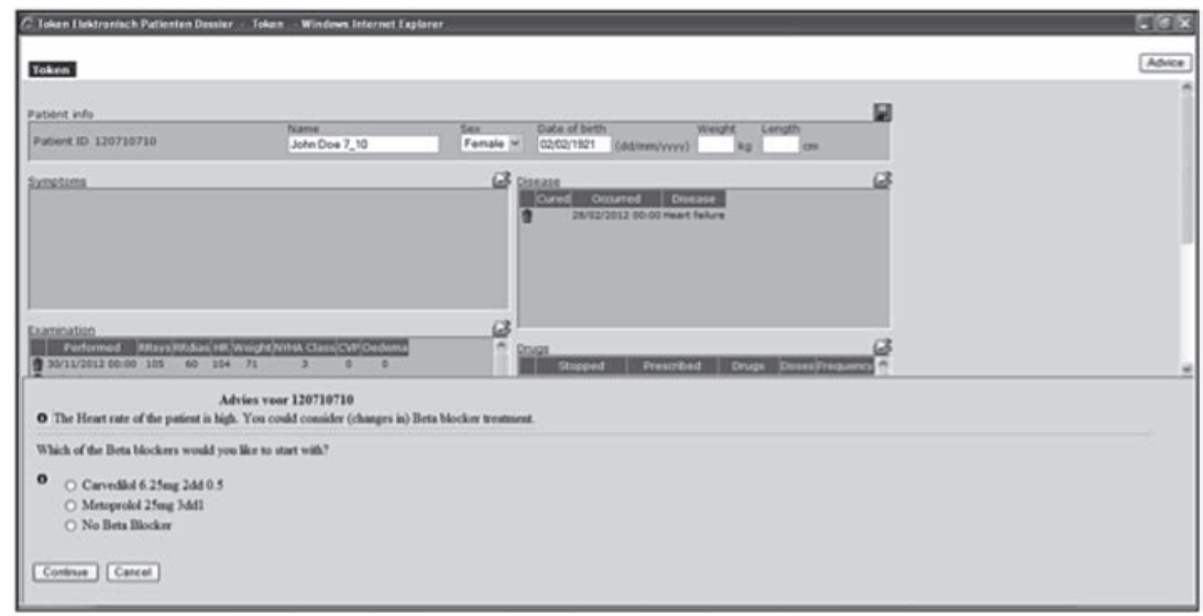

FIGURE 5.7 Electronic Patient Record with advice

We verified each flowchart using the value combinations for variables as explained above when there are two or more conditions. We selected flowchart after flowchart and tested node after node. The flowcharts in GASTINE are created in such way that it is clear to the system which one is the main flowchart; this is the starting point for the system. In this main flowchart other flows exist and along these lines they create a workflow. As an example we show how we verified the flowchart presented in Figure 5.1. It will be apparent that the method is time consuming and has to be carried out accurately.

By entering the relevant data into the EPR we make sure that the system arrives at the flowchart shown in Figure 5.1: GASTINE starts with the main flowchart "Heart failure" (main intention), from there it enters the $2^{\text {nd }}$ flowchart that is the "Fluid retention" subintention, and then it passes via decision steps to the sub-flowchart "Decrease diuretics check". To test every node of this flowchart we force the system, by entering the right data into the EPR (both historical and current data), to go to the first decision step of this flowchart. Then we carry out the twelve following steps.

1 No diuretics are entered into the EPR and then it is checked if the outcome of the decision step "Are diuretics prescribed today" is "No".

2 We then check if the system immediately goes via the next decision node "Is the lowest dose stopped (today)?" to the outcome "No". This outcome can be easily seen on the interface of the EPR, where the following recommendation "Decrease or maintain the dose of diuretic" should be present.

3 We enter into the EPR the data that is needed to test the node "Is the lowest dose stopped (today)?" providing the outcome "Yes". To this end we enter one of the three possible minimum doses of diuretics: Furosemide $40 \mathrm{mg} 1 \mathrm{dd} 1$ and its StopDate 
with the date of "today" and we check if the system's outcome is "Yes." We then delete this medication from the database.

4 We change the StopDate into a random date in the past, run the system, and check if the system's outcome for the node "Is the lowest dose stopped (today)?" is "No" this time.

5 We repeat step 3 and 4 for the other two possible minimum doses of diuretics: Furosemide $40 \mathrm{mg} 2 \mathrm{dd} 1$ and Bumetanide $1 \mathrm{mg} 1 \mathrm{dd} 1$. After receiving positive tests results, the medications are deleted from the database.

6 We now prescribe the correct diuretics, with the dose lower than the one prescribed during a previous patient-physician encounter, and check if the system's outcome after going through the first diamond of the flowchart is "Yes".

7 We also check if the system's outcome for the node "Is prescribed diuretic according to the protocol?" is "Yes" as well.

8 We check if the system's outcome for the next node "Is the dose of diuretic decreased?" is "Yes" as well.

9 We change in the EPR the dose of the prescribed medication to the same dose as the one prescribed during the previous encounter. We run the system and check if this time the system's outcome for node "Is the dose of diuretic decreased?" is "No".

10 Immediately we can see if the system's outcome for node "Is the dose of diuretic maintained?" is "Yes".

11 We change in the EPR the dose of the prescribed medication to a dose higher than the one prescribed during the last encounter. We run the system and check if the system's outcome for node "Is the dose of diuretic maintained?" is "No".

12 As last check for this flowchart we delete the diuretics prescribed today and we prescribe a diuretics that is not known by the guideline and check if the system's outcome for the node "Is prescribed diuretic according to the protocol?" is "No".

The golden standard in this case is the formalised guideline before it was entered into the system. It is assumed that the formalised guideline is correct.

\subsubsection{Most common inaccuracies}

We observed three kinds of errors during verification.

1 Errors made in the criteria logic during the creation of the flowchart via the editor; those errors were always easily corrected (e.g., value "higher than 5" instead of "higher than or equal to 5 " or assigned value being 55 instead of 5.5).

2 Errors due to incompleteness of the protocol. Those errors had to be discussed within the expert's team including professionals from different fields. Sometimes the solution of the problem resulted in changes in the flowchart of the DSS. For example, the use of the creatinine value when the NTproBNP value was not available 


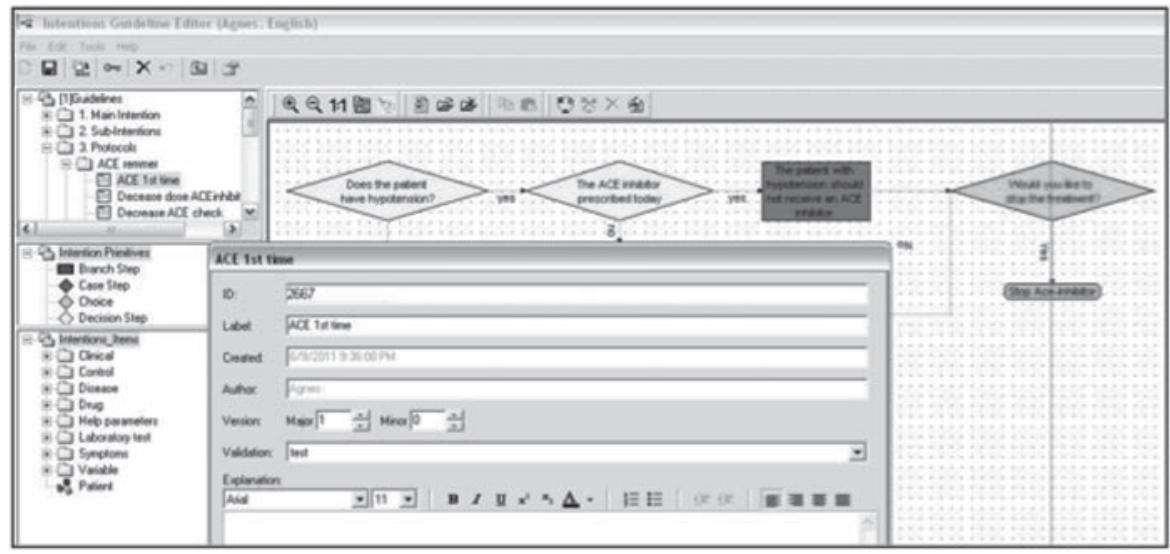

FIGURE 5.8 Flowchart ID

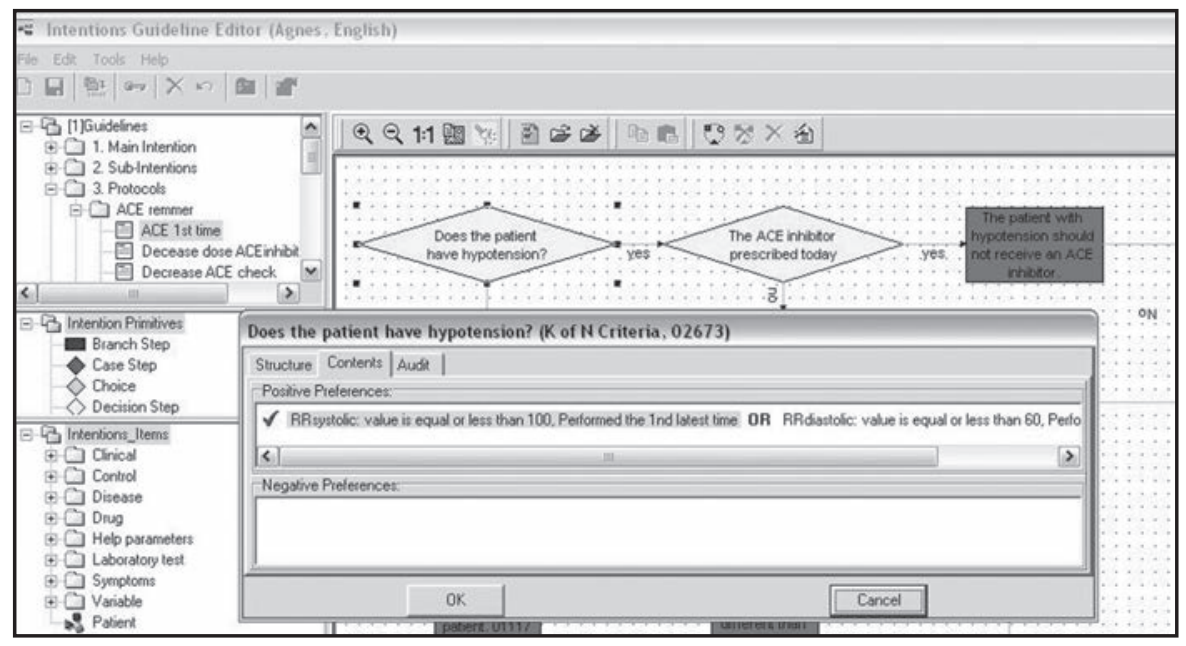

FIGURE 5.9 Primitive ID

was not originally part of the protocol. Both the protocol and the flowchart had to be changed. Because the earlier tests of the "Fluid retention" flowchart did not consider creatinine as one of the variables the tests therefore had to be repeated.

3 A programming problem (we call it the "Root problem") that occurred when changing the flowcharts. It occurred when the first primitive of the flowchart was deleted or moved. When formalizing the guideline with the Editor every folder, every flowchart and every primitive obtains its own unique ID. When the formalization is finished, the guideline is compiled and exported to a text file and to the runtime files. In Figure 5.8 we show the properties of the flowchart named "ACE $1^{\text {st }}$ time" (visible in the left top window). In the pop-up window in the middle of the screen we read the ID: 2667. In Figure 5.9 the content of the first primitive in this flow is shown and its 
ID is 02673 . We say that primitive 02673 is the root of the flowchart 2667, in other words whenever flowchart 2667 is called, primitive 02673 will be the first step that GASTINE will examine.

In the text file it can be seen (Figure 5. 10) that flowchart 2667 with label ACE $1^{\text {st }}$ time has a root primitive with ID 02673.

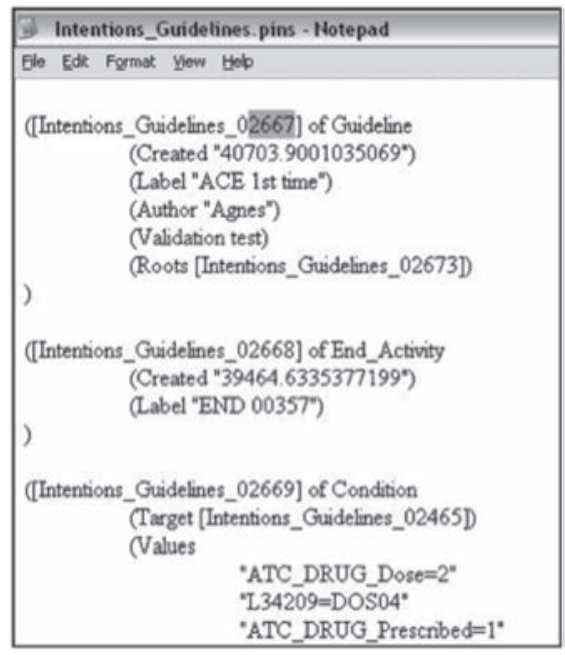

FIGURE 5.10 Guideline presented in the text file.

We observed that in a quite special situation when a new primitive was added to the flowchart as the first primitive (and therefore placed before the primitive with ID 02673), this new situation was not correctly saved in the text file. We saw that in the text file two roots were present although all connections in the Editor were correct. As a consequence when flowchart 2667 was called, it randomly jumped to one of the two roots which was observed during the tests as a random correct/incorrect answer. It was a programming bug that had to be fixed before further tests could be carried out.

\subsection{Discussion and conclusion}

In this chapter we report how we verified GASTINE. Since the code of GASTON underlying GASTINE was checked many times, we only checked whether the conditions extracted from the guideline were entered correctly into the system. Therefore, we tested for each of the sub-flowcharts whether combinations of different values of the variables used in the conditions of each decision step of the guideline led to the right recommendations. 
The values of the variables were chosen such that each condition obtained both the value True and False. We followed the strategy of choosing values as explained by Sailors. However, it was sufficient to take two values for a variable instead of three as mentioned by Sailors: one below or above the threshold (depending on the relational operator) and one equal to the threshold in such a way that the condition was assigned the value False for one value and the value True for the other. Probably the difference is caused by the fact that we can trust the code of the primitives, because they have been used in many applications.

Structuring the guideline according to the different intentions leads to a number of sub-flowcharts that use a limited set of conditions and variables. So we did not have to test all value combinations of all variables simultaneously which reduced the number of combinations to be tested considerably. Because the code of the GASTINE primitives were correct we did not have to use all combinations as required by the MCC method. This reduced the number of combinations even further. In total we tested 2414 combinations which is a much smaller number than the number of combinations that would have to be tested if the guideline was not structured by the use of intentions. In the latter case millions of combinations would have to be tested. Owing to our procedure, we could test GASTINE exhaustively. The MCC method is time consuming but in a period of seven weeks all the relevant flowcharts could be checked and where needed corrected.

Based on the results reported we may conclude that after the verification and the correction of the detected errors the system was built right. In the next chapter we will describe the validation of the system. 


\section{References}

1 Sargent R. Verification and validation of simulation models. Simulation Conference (WSC), Proceedings of the 2010 Winter. p. 166-183.

2 Preece A. Validation of knowladge-based systems: The state-off-the-art in north america. Journal of communication and cognition - Artifitial intelligence. 1994;11: 381-413.

3 Gorgels A, Takx-Kohlen B, Rutten F. Diagnose en therapie 2011; Hart- en vaatziekten: Bohn Stafleu van Loghum; 2011.

4 de Clercq PA, Hasman A, Blom JA, Korsten HH. Design and implementation of a framework to support the development of clinical guidelines. Int J Med Inform. 2001 Dec;64(2-3):285-318.

5 Latoszek-Berendsen A, Talmon J, de Clercq P, Hasman A. With good intentions. Int J Med Inform. 2007;76(3):440-446.

6 Sailors RM, East TD, Wallace CJ, Carlson DA, Franklin MA, Heermann LK, et al. Testing and validation of computerised decision support systems. Proc AMIA Annu Fall Symp. 1996:234-238. 


\section{GASTINE: Intention-based DSS in practice; results of a validation test}

GASTINE: Intention-based DSS in practice; results of a validation test. Latoszek-Berendsen A, Boyne J, Gorgels A, van den Herik J and Hasman A. 


\section{Abstract}

\section{Objective}

To validate GASTINE, in particular to test whether the proposed architecture for a DSS using intentions actually provides the envisaged support. Assuming the intentions underlying the actions are included in the formalised CIG, we hypothesise that the DSS will be able to provide more flexible advice, give less unnecessary warnings, and provide more insight into the reasons behind the generated advice.

\section{Methods}

To validate the system we used the data of 7 patients with 70 patient-physician encounters in total. Patients were chosen randomly from the group of patients treated by the specialised heart failure nurses. Validation was carried out in two phases. In phase one, for each patient-physician encounter, two items were entered into the Electronic Patient Record system interfaced to GASTINE: (1) laboratory and physical examination results and (2) the medication history of the patient. Based on the entered data, the DSS generated advice. The system was tested in both the pro-active and the re-active mode. In the pro-active mode the system generated advice based on historical data; in the re-active mode the system determined whether the prescribed medication was according to the guideline, and if that was not the case, generated warnings. Those advices and warnings were stored in a specifically created database. In phase two, a cardiologist together with a heart failure nurse practitioner appraised and compared the treatment advice generated by the system and the original treatment. For this procedure, they made use of backtracking information.

\section{Results}

In $99 \%$ of the 70 cases it was clear why GASTINE gave explicit advice i.e., an advice and an explanation. In $94 \%$ of the 70 cases the explanation was considered as more useful than the advice on its own. In $24 \%$ of 58 selected cases (criterion is NYHA class in accordance with a guideline) the system's advice and the given treatment perfectly matched and in the remaining $76 \%$ they did not correspond. In $25 \%$ of 44 selected cases (criterion is mismatch of advice and given treatment) the tester indicated that the treatment given in practice was adequate, although not in accordance with the guideline; however, for the remaining $75 \%$ of those 44 cases the tester indicated that the treatment given in practice was not optimal.

\section{Conclusions}

The use of intentions (1) gives healthcare providers more freedom and (2) distracts them less. The reason is that advices, warnings, and alerts will be given only when the users ask for it or when it is really necessary. Intentions also improved the insightfulness of the given advice by the delivery of backtracking information. 


\subsection{Introduction}

Healthcare is increasingly supported by clinical practice guidelines (CPG). The development of these guidelines is a response to (1) the discovery of large, unexplained variations in the practice of physicians, (2) the documentation of significant rates of inappropriate care, and (3) an interest in managing healthcare cost [1]. With time progressing, increasingly more CPGs are created. Therefore, it became difficult for the physicians to keep an overview of all guidelines. The CPGs are beneficial for physicians because they provide an adequate explanation of available treatments with recommendations for those physicians who are uncertain about how to proceed. The guidelines may also improve consistency of care and help to change the attitude of practitioners who became used to their, maybe outdated, medical practice. Guidelines help to structure the treatment of patients more adequately and make it more clear how to proceed, but they can harm, e.g., (a) if they do not provide adequate advice or (b) when the inadequate consensus statements are turned into performance indicators or (c) other tools inadequately critique the quality of the physician's care $[2,3]$.

Early guidelines were often based on consensus among experts from the same discipline. Gradually, this changed to consensus among experts from different disciplines, resulting in multidisciplinary guidelines. However, guideline adherence is still low. The reasons for that behaviour are related to (1) the guideline: e.g., the guidelines could contain ambiguous text or even inconsistencies; (2) the workflow: e.g., paper-based guidelines are difficult to use in clinical practice; (3) the user: e.g., physicians consider the use of guidelines as cookbook medicine, or physicians are not convinced that the use of guidelines would lead to better care; and (4) the organisation: e.g., the necessity of local adaptation.

As mentioned above, CPGs may contain ambiguities and inconsistencies. Moreover, consensus does not mean that always the best actions are suggested. Therefore, guideline recommendations are increasingly based on evidence instead of consensus. Research findings are translated into actionable recommendations for clinical care. Since the CPGs were difficult to use in practice the use of computer-interpretable guidelines ( $\mathrm{ClGs}$ ) was considered an alternative.

The core characteristic of every guideline approach is its underlying model which must be able to represent various kinds of guidelines that differ considerably in complexity and in level of abstraction. Examples of guideline modelling approaches are: PROforma, GLIF, GUIDE, PRODIGY, EON, and Asbru. Decision Support Systems (DSSs) can be used in a pro-active and a re-active way. In the first mode DSSs suggest actions that have to be carried out by the physicians whereas in the second mode the actions of the physicians are monitored and when the actions deviate from those suggested by the guideline, the physician is warned. Overviews of computer interpretable guideline formalisms 
were presented by de Clercq et al. [4], by Isern and Moreno [5], and by Sonnenberg and Hagerty [6].

But also the use of CIGs is not free of obstacles. We mention three of them. A first obstacle is that in the re-active mode DSSs lack the flexibility to accept actions that are in the spirit of the guideline. A second obstacle is that most DSSs do not inform the user of the guideline about the steps that led to the advice. A third obstacle is that as soon as the DSSs would present the guideline-driven steps to the user, it is difficult to understand the rationale of why the steps should be taken, because the goal of the treatment is not yet available. Facing the obstacles, we proposed in an earlier article an approach that attempts to resolve the three issues. We designed and implemented an advanced functionality, as an extension to the existing DSS GASTON [7]. When working in the re-active mode, GASTON is executed in such a way that it is able to accept actions that are in the spirit of the guideline. This was established by the use of intentions in the guideline description. An intention is a high-level goal description that is abstract and not quantified. An example of an intention is 'Fluid retention'. The intentions - seen as a determination to act in a certain way or to do a certain thing - therefore make clear to the user why the various steps via the guideline were taken. Thus, the use of intentions makes the guideline easier to interpret and therefore also easier to update. In total six different intentions were recognised in the heart failure guideline: (1) the "Reduce afterload" intention mainly concerning medications from the group of ACE inhibitors and Angiotensin-II receptor antagonists; (2) the "Fluid retention" intention mainly concerning medications such as diuretics and spironolacton; (3) the "Increase ejection fraction" intention supporting Betablocker prescription; (4) the "Kidney function" intention monitoring kidney parameters; (5) the "Increase contractility" intention concerning the titration of digoxin medication and (6) the "Preload reduction" intention concerned with carrying out the titration of nitrate. The new extension was called GASTINE (GASTon INtentional Expression) [8]. Here we remark that Asbru also uses intentions. However, our approach differs in the sense that our system checks whether the user acts according to the guideline and if not it checks whether the action is according to the spirit of the guideline. If we understand the literature about Asbru correctly this last feature is not implemented in Asbru although the authors stated that the system should be able to do so with the help of plan recognition [9].

For our research, we hypothesise that when the intentions underlying the actions are included in the formalised CIG, (1) the DSS will be able to provide more flexible advice, (2) give less unnecessary warnings, and (3) provide more insight into the reasons behind the generated advice. By advice we understand a recommendation concerning a future action that is based on the evidence-based guideline. Further in the text we also will 
use the term warning (cautionary advice, indicating a possible danger or problem) and alert (mostly presented in our system as pop-up, a quick note about the presence of a dangerous situation).

In this contribution we investigate whether using intentions makes the execution of guidelines more flexible (allows the system to recognize actions not according to the guideline but in its spirit), and more insightful [10].

\subsection{Validation tests}

The goal of the validation is to answer the following three questions.

1 Do intentions actually provide more flexibility when critiquing medical decisions?

2 Do intentions help to better understand the reasons underlying an advice?

3 Could the system be useful in practice by preventing deviations from the guideline? To validate the system we used the data of 7 patients with in total 70 patient-physician encounters $(8,12,17,7,5,10,11$ encounters per patient, respectively). Patients were chosen randomly from the group of patients treated by the specialised heart failure nurses (HF nurses). All patients were diagnosed and treated for heart failure, often in combination with other diseases. Because only the heart failure guideline was implemented, we randomly selected patients from those by whom other cardiac conditions are under control and not considered by the guideline used in our tests. The heart failure guideline was selected because of its complexity. During these validation tests we used the treatment part of the guideline in combination with the titration protocols for heart failure medications designed at the cardiology department of the Academic Hospital Maastricht, the Netherlands [11]. The patient - physician encounters took place every two weeks somewhere between 2005 and 2009. The validation was carried out in two phases.

\subsection{Phase one}

In phase one, for each patient-physician encounter, two items were entered into the Electronic Patient Record system interfaced to GASTINE: (1) laboratory and physical examination results and (2) the medication history of the patient. None of the selected patient-physician encounters concerned the start of a treatment. The reason is that the treatment starts in the heart failure outpatient clinic (OPC) or in the hospital. The medication is prescribed to the patients there. After that, the patients are taken over by the HF nurses and treated in the home situation. A HF nurse is a specialised nurse 
who adjusts medication, in consultation with the cardiologist, certainly for diuretics, sometimes for ACE inhibitors and Beta-blockers.

The data available to us came from the recordings of the HF nurses. The data from the hospital system were not available to us due to the transition to a new Hospital Information System (HIS). The first record of each patient in our EPR dealt with an already started treatment. This provided problems to our system since it considered the first record as the first visit. We call this situation "the $1^{\text {st }}$ visit issue". A more detailed explanation about this $1^{\text {st }}$ visit issue is presented in subsection 6.5.2.

The information about this first record includes only:

1 Actual (not older than 7 days) laboratory data,

2 Actual (not older than 1 day) physical examination data,

3 In the pro-active mode - neither the list of prescribed and stopped medications, nor medications prescribed during the current encounter; in the re-active mode - only the list of medications prescribed during the current encounter.

For the following patient records the information also included:

1 Historical laboratory data,

2 Historical physical examination data,

3 In the pro-active mode - the list of prescribed and stopped medications (excluding the one(s) prescribed during the current encounter); in the re-active mode - the list of prescribed and stopped medications including the one(s) prescribed during the current encounter.

Based on the entered laboratory and physical examination data and (if available) previous active and non-active medications and new prescriptions, the DSS generates advice. This advice, always concerning the prescription of medications, together with information about the way the systems traversed the flowchart in this encounter, was stored in a specifically created database.

The system was tested in both the pro-active and the re-active mode. In the pro-active mode the system generates advice based on historical data, in the re-active mode the system determines whether the prescribed medication was according to the guideline and if that was not the case, generates warnings.

\subsection{Phase two}

A cardiologist (A.G.) together with a heart failure nurse practitioner (J.B.) evaluated both the treatment advice generated by the system and the original treatment. First, the nurse practitioner carried out the validation, then the cardiologist assessed her judgements and when he did not agree discussed the judgement with the nurse. In these cases they 
together reached consensus. When they stated that the advice of the system was not according to the guideline, the guideline was checked to see whether they were right. They both had not been involved in the development of the system. They did, however, answer questions about the interpretation of the guideline during the formalisation process. From now on we will refer to them as the testers.

To make the interpretation of the validation easier, we created a questionnaire that the testers had to fill in for each of the 70 encounters. The following questions were asked.

1 The system gives an explanation of how it did proceed to generate an advice. On the basis of the explanation do you understand why this advice was given?

2 Is the explanation more insightful than only the advice?

3 Is the advice given in accordance with the guideline?

4 If the advice is not according to the guideline, what advice should have been given?

5 Was it clear from the explanation of the system why an advice different from the one according to the guideline was given?

6 Does the correct advice of the system correspond to what was done in practice?

In Figure 6.1 we show how the advice was presented to the user. The advice is generated in the pro-active mode in a situation without Beta-blocker treatment where the patient has a heart rate higher than $70 \mathrm{bpm}$. The system offers a choice either to start Betablocker treatment with one of the medications suggested by the heart failure protocol or to postpone Beta-blocker treatment. The heart failure guideline states that a Betablocker is commonly added in a later stage of the treatment; however, there is no information indicating when exactly it has to be started. Therefore, the system offers the choice mentioned above: the care provider has to decide what the best moment is to start the treatment.

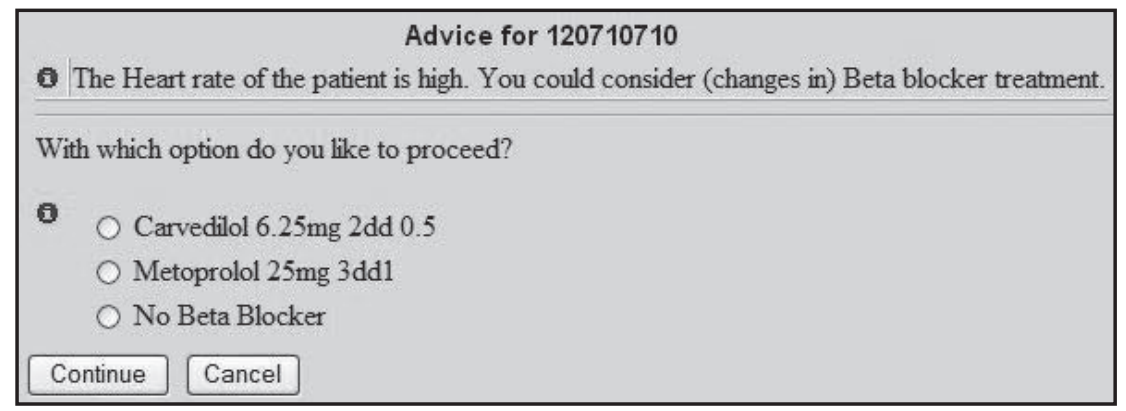

FIGURE 6.1 The advice given in pro-active mode.

Next to the given recommendation an " $\mathrm{i}$ " symbol is visible. By clicking on this round button the tester obtains more information concerning the given advice, called by us backtracking information. In Figure 6.1 there are two symbols " $\mathrm{i}$ " present. The first " $\mathrm{i}$ " 
shows the path that the system took through the guideline to generate a warning about the high heart rate; the second " $i$ " shows the path that the system took to generate the advice about the Beta Blocker medication. The Beta Blocker treatment is just one step further in the guideline than the warning about the high heart rate and therefore the path descriptions are identical. This may seem superfluous. However, we enable backtracking for every step that communicates with the user (e.g., choice step, recommendation) because some steps in the flowchart may be reused in different parts of the guideline and consequently the same step can be reached via different paths.

In the re-active mode all backtracking information is available as well as a possibility to switch to pro-active mode, see Figure 6.2.

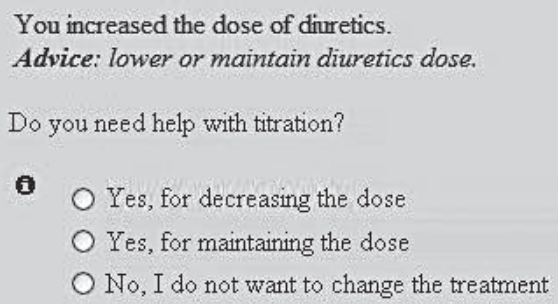

FIGURE 6.2 A warning generated in re-active mode.

We recognize the re-active mode by the way the warning is given. First, the action(s) taken are mentioned, then advice about the correct action is given and finally the option to follow the system's advice (and switch to the pro-active mode) is mentioned together with the possibility to continue treatment as planned.

In Figure 6.3 we show how the backtracking information is presented. We present here the backtracking information that belongs to the advice from Figure 6.1. The text is created using one of a fixed set of sentences. The combination of (1) the type of step that was executed (in this case a decision step) and (2) its label together with its outcome - true or false - creates a sentence. For example, every "decision step" primitive corresponds to the sentence Because - decision step - "Label" is "Value".

To satisfy the intention, the "Increase ejection fraction" (sub)intention is executed

Because - decision step- "The heart rate is more than $70 \mathrm{bpm}$ " is True.

Because -decision step- "Does the patient have oedema? (based on BNP)" is False.

Because -decision step- "Is Oedema $>0$ ?" is False.

Because -decision step- "Does the patient receive Beta blocker treatment?" is False

To satisfy this, strategy "Prescribe Beta blocker" is executed

Explanation: The recommended action is "The Heart rate of the patient is high. You could consider (changes in) Beta blocker treatment." 
First, it is explained what the intention is that needs to be fulfilled (increase ejection fraction) and then the four executed decision steps are shown. The outcome of every decision step is determined by criteria. If the criteria are met then the outcome of the decision step has the value "True", otherwise it has the value "False". After the list of the relevant decision steps (see Figure 6.3), the system mentions the plan (strategy) and the recommended action that should be carried out to fulfil the intention. The advice is generated and the choice of possible medications is presented to the care provider as shown in Figure 6.1. This advice can also be requested in the re-active mode.

\subsection{Results}

In this chapter we aim to answer the first two questions of section 6.2. For this purpose, we analyse the answers to the questions in the questionnaire that we used for validation. The answers provided us with information about the quality of the given advice and warnings, its accuracy, and its usefulness. The tester not only had to compare the advice of the system and the actually given treatment with the guideline's advice, but was also asked to evaluate the quality of the explanation belonging to the advice, and to provide suggestions for improvement if necessary.

During data entry into the EPR we discovered that for 9 patient-physician encounters the NYHA (New York Heart Association) class was missing. The treatment of the patient depends on the seriousness of the heart failure: without information about the NYHA class the guideline cannot be executed. However, this does not influence the interpretation of

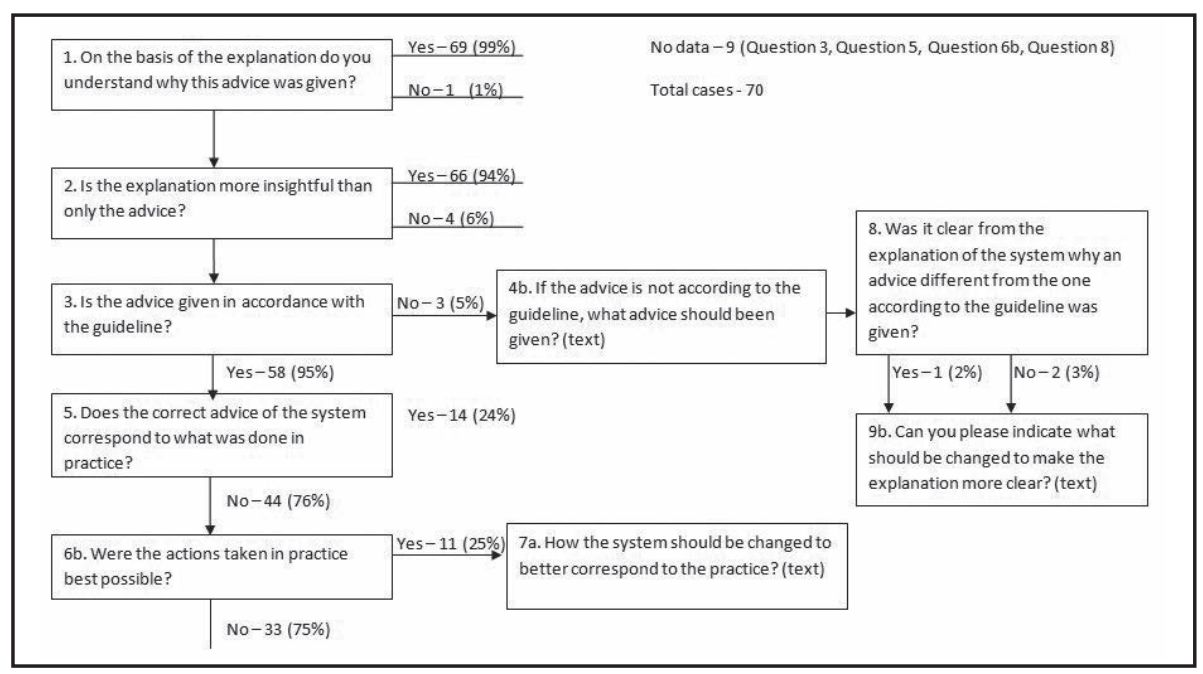

FIGURE 6.4 The overview of answers to the questions from the questionnaire. 
the next record because in this record all historical information is again available. For those 9 cases only questions 1 and 2 could be answered.

In Figure 6.4 we present an overview of percentage of answers "Yes" and "No" to the questions from the questionnaire. The percentage mentioned in question 3 is, due to the above mentioned reason, based on 61 encounters.

\subsubsection{The system gives an explanation of how it did proceed to generate an advice. On the basis of the explanation do you understand why this advice was given?}

In $99 \%$ of the 70 cases the answer was "Yes". In one case the answer was "No", caused by insufficient information regarding the "side effects". After analysis of the guideline, it was clear that the advice of the system was correct. However, when a side effect occurs, the user would like to read in the explanation which side effect it is.

\subsubsection{Is the explanation more insightful than only the advice?}

The answer "Yes" was given in 94\% of the 70 cases. The four "No's" were related to four distinct causes.

(1) The $1^{\text {st }}$ visit issue occurs when the system is expected to give advice based on only one laboratory test and one set of physical examination data. Let us explain the problem using an example of ACE inhibitor advice given in one of our cases. In this case we had a patient who already has been diagnosed and treated for heart failure in the OPC. Then the team of the HF nurses took the patient over. The patient record data available to our system looks as follows: examination data from less than $24 \mathrm{~h}$ ago; a laboratory test outcome from less than 3 days ago; no medication data available. In the pro-active mode the system advised to start with ACE inhibitor treatment and suggested the starting doses of one of the medications. When the tester compared this advice with the actions taken in practice, (s)he sees that in practice the patient received the maximal dose of one of the ACE inhibitors. The backtracking information contains sentences such as: "Because -decision step- "Oedema - occurred for the first time" is True" or "Because -decision step"Patient receives ACE inhibitor treatment" is False". These sentences indicate that the system considers these data to belong to a new case of heart failure. In the re-active mode, our system warned that according to the EPR the ACE inhibitor is given for the first time and therefore advised not to use the maximal dose but one of the starting doses. Here we note the following: if in the EPR it was recorded that the patient already received the highest doses of the ACE inhibitor and that there were no side effects, the system would advice maintenance of the dose of ACE inhibitor. That was the advice that the tester expected. The tester experienced the advice as confusing and was not able to figure out from the backtracking information what exactly the issue was, because this 
information only explained how the system had arrived at the advice and did not state explicitly that the system regarded the data as coming from a first visit. This situation happened for the $1^{\text {st }}$ visit encounter of the second patient (for the $1^{\text {st }}$ visit of the $1^{\text {st }}$ patient the NYHA data was not available, therefore no treatment advice was given) and the tester contacted us for an explanation assuming something was wrong with the system. After the explanation of the $1^{\text {st }}$ visit phenomenon, they understood why the difference occurred and proceeded with the next encounters. The other $1^{\text {st }}$ visit records were now interpreted correctly by the tester.

(2) There is not sufficient information in the backtracking information, e.g., the fact of not indicating which side effect occurred as mentioned above.

(3) Missing an expected advice due to the use of normal values. According to the guideline, e.g., a normal value for potassium lies in the range between $3.5[\mathrm{mmol} / \mathrm{L}]$ and $5.5[\mathrm{mmol} / \mathrm{L}]$. In this case the patient had a potassium level around $5[\mathrm{mmol} / \mathrm{L}]$ and the system gave advice according to the guideline. However, in practice sometimes the guideline recommendations are not followed because such a potassium level is considered as high.

(4) The use of a range of normal values with strict boundaries. In this case it was not clear to the tester why the advice to change a therapy was given for a "stable" patient. According to the guideline the norm for creatinine for a man is $110\left[\mathrm{ml} / \mathrm{min} / 1.73 \mathrm{~m}^{2}\right]$ and for a woman $90\left[\mathrm{ml} / \mathrm{min} / 1.73 \mathrm{~m}^{2}\right]$. In this case the patient was a woman and three creatinine values concerning three patient-physician encounters were available. Those three values were 93, 95, $96\left[\mathrm{ml} / \mathrm{min} / 1.73 \mathrm{~m}^{2}\right]$, thus above the norm. In accordance with the guideline, GASTINE takes previous values into account and concludes that the creatinine value is already too high and still rises. We understood from the comments of the tester that in practice the described situation belongs to a "stable" patient and therefore no change in treatment would be necessary.

\subsubsection{Is the advice given in accordance with the guideline?}

In $95 \%$ of the 61 encounters the answer was "Yes". But, there were still three cases where the tester indicated a deviation from the guideline. The question is whether the given advice was indeed not according to the guideline or just not according to what is done in practice.

The first of the three cases concerned the situation described in point (3) of subsection 6.5.2. The system correctly advised spironolacton (a potassium-sparing diuretic) for a patient with NYHA class 3 . In practice, the patient's potassium value is considered too high for adding spironolacton.

In the second case the system advised "decrease or maintenance of the diuretics". Both, decrease and maintenance were possible and correct actions that could be carried out 
(but probably not for every patient, it depends on the data). The system suggested a decrease of diuretics because of a too high creatinine value. However, maintenance of the dose would be correct as well, since the patient still had oedema. In the pro-active mode the system therefore suggested both options; however, the tester focussed on the oedema value and therefore incorrectly concluded that the advice was not according to the guideline. In the re-active mode no warning was generated since the dose of diuretics was maintained for the patient.

The third case concerns a different issue with respect to the treatment by diuretics. The implemented flowcharts take both the physical examination result Oedema and the laboratory value of Central Venous Pressure (CVP) into account. When either parameter indicates oedema it triggers a check of the medications. In this particular case both the laboratory result and the physical examination Oedema result did not indicate the presence of oedema. The fact that the patient gained $5 \mathrm{~kg}$ in weight within 2 weeks was considered by the tester as an indication of oedema. The weight is not used in the guideline and that was the reason that the system's advice was not accepted by the tester. When the answer to the questions discussed in this subsection was "No", the question of subsection 6.5.4 had to be answered, otherwise the question of subsection 6.5.6.

\subsubsection{If the advice is not according to the guideline, what advice should be given?}

As mentioned in the previous subsection the tester stated that in $95 \%$ of the 61 encounters the system's advice was according to the guideline.

The tester provided us with multiple suggestions about the form of the advice which GASTINE should give to make it more accurate. The remarks were given in free text.

The suggestions were related to: (1) the use of additional tests (such as weight, or the use of the creatinine value when the N-terminal prohormone of brain natriuretic peptide (NTproBNP) value is not available). NT-proBNP is an important parameter because its level in the blood is used for screening and diagnosis of acute congestive heart failure (CHF) and may be useful to establish the prognosis of heart failure; (2) the addition of a formalised concept of a "stable" patient as mentioned in subsection 6.5 .2 ; (3) the adaptation of normal values such as for the example of potassium is mentioned in subsection 6.5.2.

There is one more case, where alternative medications were used. By alternative medications we understand medications that belong to the same drug category or medications that have the same effectiveness and can be exchanged. A medication can have more than one purpose so that it can be used in different situations (e.g., paracetamol is used as pain reliever and fever reducer); if an alternative medication has the same purpose as the medication prescribed by the guideline, the alternative medication is according to the intention of the (sub)guideline. A titration protocol is necessary to give detailed information about the dosage of the alternative medication; 
however, the system can still work if the titration protocol is not yet inserted. In cases where, in practice, alternative medications were prescribed, the system gave general advice about the treatment (e.g., decrease/ increase the dose, stop medication) when the relevant titration protocol was not yet incorporated in GASTINE. The alternative medication, of course, had to be already known to the system together with its purposes. If one of these purposes matches the intention of the guideline, the medication, although it is not according to the guideline, is accepted.

\subsubsection{Was it clear from the explanation of the system why an advice different from the one according to the guideline was given?}

As mentioned in subsection 6.5.3 there were, from the point of view of the tester, three cases where the system deviated from the guideline. But, only in one of the three cases it was understandable for the tester, based on the backtracking information, why this difference occurred. That was for the case with "too high creatinine", explained in subsection 6.5.2.

In case of "gaining weight" and "spironolacton" it would help when the backtracking information also showed the criteria that decide about the outcome of a decision step. We will illustrate why the results of the criteria are also important for understanding the advice using the example of determining whether the ACE inhibitor causes side effects.

While building the flowchart we use labels that describe each step for easier navigation, see Figure 6.5. The primitive in the middle of this figure has the label "Does the patient have side effects caused by the last dose of the ACE inhibitor?" The label is used for displaying the backtracking information as is clear from Figure 6.3 for another case. In the case of Figure 6.5 the corresponding sentence in the backtracking information would be Because - decision step - Does the patient have side effects caused by the last dose of the ACE inhibitor? is True.

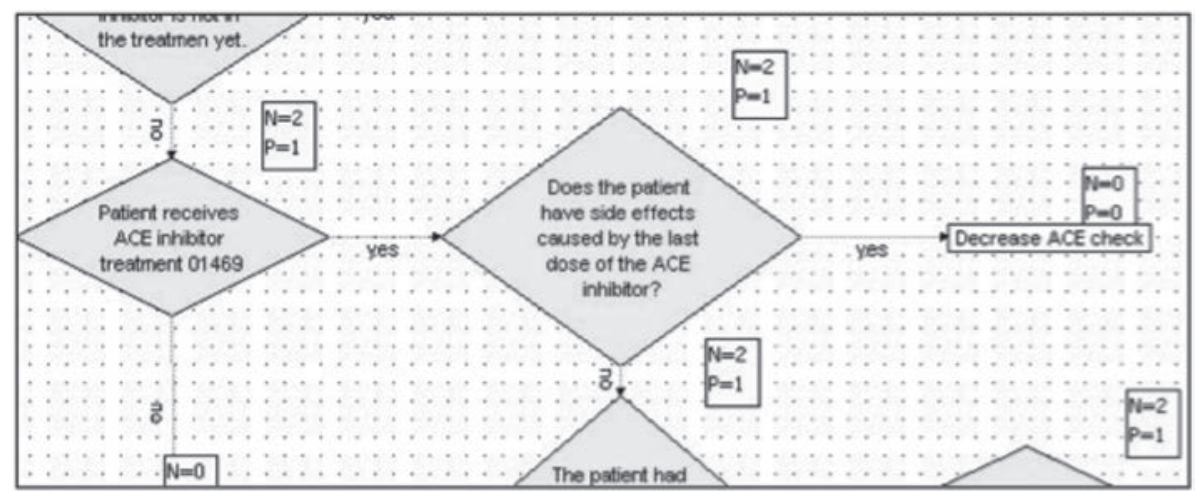

FIGURE 6.5 The ACE-inhibitor flowchart; checking side effects. 
The outcome of this decision step is determined by criteria, see Figure 6.6. There are several criteria that mention specific side effects and the system checks if any of them is present.

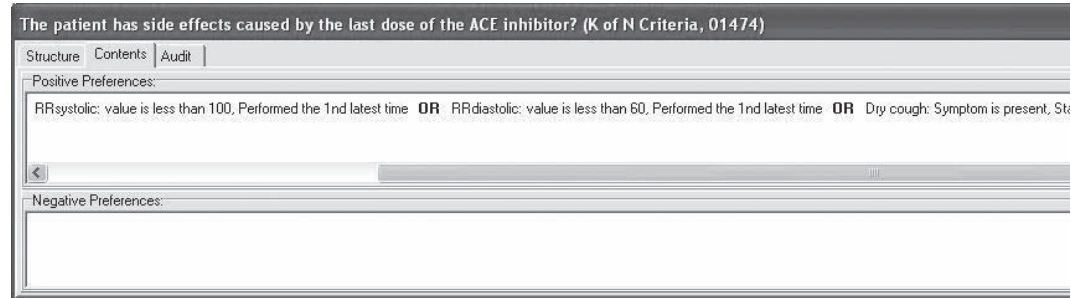

FIGURE 6.6 The decision step in the ACE-inhibitor flowchart that uses side effects for its determination.

When the backtracking information is generated, the outcome of the criteria is not listed. The user can see that there are side effects caused by the ACE inhibitor but cannot see which one(s). However, the tester would like to see which side effect was present. This means that the backtracking information should be even more detailed and show the outcome of a number of sub-steps related to the decision step.

In the case of "gaining weight" and "spironolacton", the addition of the criteria to the backtracking information would show to the tester that weight is not considered as one of the criteria or that the system uses normal values for potassium in the range between 3.5 to $5.5[\mathrm{mmol} / \mathrm{L}]$ to generate the advice.

\subsubsection{Does the correct advice of the system correspond to what was done in practice?}

In $24 \%$ of the 58 cases the system's advice and the given treatment perfectly matched and in the remaining $76 \%$ they did not correspond.

In $25 \%$ of the 44 cases where the system's advice and the given treatment did not match, the tester indicated that the treatment given in practice was adequate, although not in accordance with the guideline; however, for the remaining $75 \%$ of those 44 cases the tester indicated that the advice given in practice was not optimal.

Most of the reasons why a system's advice could lead to a better treatment were the addition or exclusion of some medications. For example, after checking all kidney parameters, GASTINE concluded in one situation, that the kidney functioned adequately. Since the patient still received medication that supports the kidney function, GASTINE advised to stop that medication. However, the tester found mismatches between the actions in practice and the advice based on the kidney function intention in three patients. In a second situation, the system concluded that the patient's heart rate was high and that 
the patient did not receive medication to treat it. Before generating the advice GASTINE checked whether the conditions to add this medication were met. There was a mismatch between the advice based on the Increase ejection fraction intention and the actions in practice in four patients.

In a third case, GASTINE warned that the medication should be stopped because of low values of the blood pressure.

\subsection{Discussion}

The validation was performed to answer two of our research questions: (1) do intentions actually provide more flexibility in making medical decisions? and (2) do they help to understand the reasons underlying an advice? In addition we now can answer the third question about the potential usefulness of the system.

\subsubsection{Flexibility}

We evaluated whether the proposed architecture for a DSS using intentions actually provides the flexibility we envisaged. We understand by flexibility the capability of the system not to react in situations where the physician does not work according to the guideline but does work in its spirit.

There is a difference in giving advice in the pro-active and the re-active mode. In the proactive mode the system does not have to check whether the advice is in the spirit of the guideline because the system's advice follows the guideline. However, in the re-active mode the warning of the system is based on incorrect actions of the healthcare provider. Since in only $24 \%$ of cases the system's advice matched exactly the treatment given, the re-active mode produced many alerts and additional advices. In the phase of evaluating the system we allowed all alerts, warnings, and advices to appear, even if the actions taken were in the spirit of the guideline (e.g., prescribing an alternative medication, see Figure 6.7). For actions that are in the spirit of the guideline, the system can be tuned not to show warnings immediately. The system could be tuned to generate a warning only after the alternative medication has been prescribed, for example 5 times, or after a certain period of time, or when a predefined value is reached by some parameter in the EPR. Not showing warnings at all as well as not showing alerts, we agreed, is not an option. The flexibility that the system gives is desired but the security of following the guideline is required as well.

As shown in the example of Figure 6.7, our system recognised that the alternative medication had the same purpose as the medication suggested by the guideline, and 


\section{Warning for 120710710}

Patient receives diuretics which are not mentioned in the protocol.

The patient still has oedema, please increase the dose of diuretics if possible.

FIGURE 6.7 Warning generated in case of an alternative medication.

allows the user to proceed without exactly following the guideline. As is clear from this example the system did not contain the titration protocol and therefore does not present more detailed information about the dosage. The use of a system such as GASTINE increases the flexibility by accepting actions taken by the physician that are in the spirit of the guideline.

\subsubsection{Insightfulness}

In $99 \%$ of the cases the system gave an understandable explanation of how it did proceed to generate an advice. In $94 \%$ of the cases the tester agreed that advice with explanation was preferred over plain advice. The backtracking information did provide more insight into why the recommendation was given. The backtracking information is clustered per (sub)intention which means that all actions related to one intention are presented below each other. Such a feedback makes it easier to oversee the steps that led to the advice. However, in practice, the tester was not fully satisfied with the backtracking information. The system provides the final result of the assessment of a decision step, but does not mention the results of the intermediate steps. The enrichment of the backtracking information with the results of these intermediate steps, as described in subsection 6.5.5, would help the user to understand why a particular advice was given, or why his actions generated the warning even better. The suggestions provided by the tester are easily implementable and will increase the insightfulness of the system even more.

Finally, we would like to underline the importance of the physician and computer working together. The healthcare provider might do something different from what the guideline suggests or patient data allows; for instance, the system gives a warning and does not accept the physician's actions, preventing him from making a mistake. The computer system collects data, checks criteria, and executes rules faster than humans, but it provides advice based on dry facts; the dry information is for the system the starting point for the treatment. In contrast, the healthcare provider is the one who can communicate with the patient, can assess his emotional state, can respond to his needs and take responsibility for the performed actions. Those two approaches combined create a very powerful tool that can improve quality of care and patient outcomes. 


\subsubsection{Usefulness}

In the study the system was evaluated, and simultaneously the actually given therapy could be assessed.

We found a considerable deviation between guideline (or protocol) advice and practice. It appeared that in $74 \%$ of relevant encounters ( $47 \%$ of all 70 encounters) actions deviated from the actions advised by the guideline. The deviations occurred consistently (in all records where this decision had to be made) in four patients. For one patient there was only one deviation from the guideline and for two patients the guideline was followed. Of the six intentions, two were not related to mismatches. These two intentions were not executed because they are related to NYHA class 4 and our patients did not have that state of disease. It is clear from the above that use of a system such as GASTINE may reduce these deviations and that therefore the system can be useful.

However, there were a number of situations where the tester indicated that the system gave incorrect advice. Does this make the system less useful? This question leads to two follow-up questions. (a) Are the deviations due to errors in the formalisation of the guideline? (b) Can the errors be corrected or are the errors in the guideline itself? We conjectured and partly observed that the deviations were due to (1) the interpretation of what is a (too) high potassium value, (2) the lack of an additional criterion in the guideline concerning the presence of oedema and (3) the system lacking a formalised concept of a "stable patient". So, may we thus conclude that the guideline was not correct or not sufficiently concrete for these situations and that it does not use the concept of stable patient? No, we need to remember that in the guideline we find general descriptions of which treatment should be given under which circumstances (e.g., "By all patients with heart failure the ACE inhibitor treatment in combination with diuretics should be considered"). In the (local) protocol we find detailed criteria that are used to determine how the treatment should be given. The more detailed the information stored in the protocol and/or our system is (e.g., criteria, alternatives), the better the advice and critique.

If the system would have been used in practice it may be expected that the number of deviations from the guideline will decrease.

In our study we noted that in 9 encounters the NYHA class was not mentioned. In case of missing data, the suggestion of the tester was that it is better to give no advice about the treatment at all, since an advice could mislead the health professional. However, a warning about missing data can be given that offers the possibility to fill in these missing data directly. After data entry the advice of the system can be generated using the enriched data set. In this way GASTINE will increase the completeness of the data.

All in all, from the tests performed and analysed we tentatively may conclude that the system is useful in practice. However, only when we test the system's use in practice will 
we be sure that the system will reduce deviations from the guideline. More research should be carried out focussing on (1) special cases caused by measurements falling near the boundaries of ranges and (2) how to cope with them when formalising a guideline.

\subsubsection{Limitations}

In this contribution we mentioned many advantages and challenges that occurred during the validation of the heart failure guideline implemented in GASTINE. Our results are based on only 7 patients with in total 70 encounters about which not always all data were available. Still, we believe that our conclusions are indicative for a larger number of patients and encounters. Deviations from the guideline occurred consistently. Although the system could have prevented deviations from the guideline in almost $50 \%$ of the encounters this does not guarantee that in practice these deviations will be prevented. It would therefore be interesting to validate GASTINE on a larger group of patients during real time encounters.

\subsection{Conclusions}

After a brief but abundant discussion we now arrive at three conclusions based on our validation procedure with GASTINE.

First, the use of intentions provides more flexibility in giving advice. By comparing the physician's purpose behind his actions with the intention(s) of the guideline, we may conclude that healthcare providers have more freedom and are less distracted because the advices, warnings, and alerts only will be given when they ask for it or when it is really necessary.

Second, intentions also improve the insightfulness of the given advice by the delivery of backtracking information. The use of intentions structures the flowchart. Via this structure it can be easily deduced (by backtracking) which intention led to the suggested new action. The backtracking shows the outcome of the decision steps and in future it can even show the outcome of the criteria that determine the outcome of the decision step. It gives the healthcare provider an explanation of how the system arrived at the advice, warning, or alert. From the study, we may conclude that backtracking is a very useful tool and appreciated by the tester.

Third, the system is expected to be useful in practice. Admittedly, many of the decisions made in practice were not optimal. However, the use of the system may reduce the number of deviations between practice and guideline to a sufficient degree. Yet, some problems concerning ranges remain to be solved. 


\section{References}

1 Shaneyfelt TM, Mayo-Smith MF, Rothwangl J. Are guidelines following guidelines? The methodological quality of clinical practice guidelines in the peer-reviewed medical literature. JAMA. 1999 May 26;281(20):1900-1905.

2 Shaneyfelt TM, Centor RM. Reassessment of clinical practice guidelines: go gently into that good night. JAMA. 2009 Feb 25;301(8):868-869.

3 Latoszek-Berendsen A, Tange H, van den Herik HJ, Hasman A. From clinical practice guidelines to computer-interpretable guidelines. A literature overview. Methods Inf Med. 2010;49(6):550570 .

4 De Clercq P, Kaiser K, Hasman A. Computer-Interpretable Guideline formalisms. Stud Health Technol Inform. 2008;139:22-43.

5 Isern D, Moreno A. Computer-based execution of clinical guidelines: a review. Int J Med Inform. 2008 Dec;77(12):787-808.

6 Sonnenberg FA, Hagerty CG. Computer-interpretable clinical practice guidelines. Where are we and where are we going ? Yearb Med Inform. 2006:145-158.

7 de Clercq PA, Hasman A, Blom JA, Korsten HH. Design and implementation of a framework to support the development of clinical guidelines. Int J Med Inform. 2001 Dec;64(2-3):285-318.

8 Latoszek-Berendsen A, de Clercq P, van den Herik J, Hasman A. Intention-based expressions in GASTINE. Methods Inf Med. 2009;48(4):391-396.

9 Shahar Y, Miksch S, Johnson P. The Asgaard project: a task-specific framework for the application and critiquing of time-oriented clinical guidelines. Artif Intell Med. 1998 SepOct;14(1-2):29-51.

10 Latoszek-Berendsen A, Talmon J, de Clercq P, Hasman A. With good intentions. Int J Med Inform. 2007;76(3):440-446.

11 Gorgels A, Takx-Kohlen B, Rutten F. Diagnose en therapie 2011; Hart- en vaatziekten: Bohn Stafleu van Loghum; 2011. 
General discussion and conclusions 
To change the clinicians' behaviour, Clinical Practice Guidelines (CPGs) should provide adequate patient-specific decision support during patient encounters, which is reliable and understandable by the clinicians. Paper-based CPGs are not easy to use in practice. That is why guidelines are implemented in a Computer Interpretable Guidelines (CIGs) formalism that provides support based on patient data. Over time, we observed an increase of guidelines implemented in Decision Support Systems (DSSs) [1-3] that help users to follow a guideline. The DSS retrieves all necessary patient data from an Electronic Patient Record (EPR) and generates patient specific advice based on these patient data and the guideline content.

The main contribution of the research presented in this thesis is the introduction of intentions and their use in the formalization of guidelines. Formalization of guidelines implies a translation of narrative guidelines using computer interpretable templates for representing guideline knowledge and using clinical abstractions that are appropriate for particular guideline sub-domains [4].

This chapter summarises and discusses our work. It presents the principal findings in section 7.1, and addresses strengths and weaknesses of our approach in section 7.2. Furthermore, in section 7.3 it relates our work to that of others, and in section 7.4 the significance of our work is explained. Finally, it provides an outlook on future research in section 7.5 and presents concluding remarks in section 7.6.

\subsection{Principal Findings}

In the introduction in Chapter 1, five research questions were posed that served as a guide for the research described in this thesis. We reiterate and address these research questions below.

\section{RQ1: What are high quality guidelines and what is the best way to create them?}

To answer that question we performed a literature study and gathered information from three different perspectives.

First, we looked from the perspective of the CPG. We established that a high quality CPG has following characteristics.

- A guideline is evidence-based.

- A guideline is valid, that means when implemented, it will lead to outcomes that it intended to achieve and that its recommendations are both internally and externally valid [5-7].

- A guideline is reliable, that means guideline users will interpret the recommendations in the same way $[5,6,8]$. 
- A guideline is reproducible, that means given the same data, other guideline producers will offer the same recommendations $[5,6,8]$.

- A guideline is developed by multidisciplinary teams and potential biases inherent of guideline development have been addressed adequately [5, 7].

- $\quad$ A guideline is produced within established guideline programs (e.g., structured and coordinated programs designed with the specific aim of producing several clinical practice guidelines) or by government-funded agencies. The research showed that guidelines created within such organisations had a higher quality than the guidelines produced by specialist societies. This is due to the fact that government-funded agencies have a guideline program that provides a systematic procedure with key elements such as a multidisciplinary guideline development group, systematic literature review, external peer review, and different products for dissemination [5, 7].

- A guideline is clinically applicable, that means individual recommendations within a guideline can be easily implemented in practice $[5,8]$.

- A guideline provides clinical flexibility [5].

- A guideline is well documented [5, 9].

Second, we looked from the perspective of a CIG. A high quality CIG should have the following characteristics.

- The underlying model - must be able to represent various kinds of guidelines that differ considerably in complexity and in level of abstraction.

- A standardized terminology - should be imposed so that the terms in the guidelines can be matched with terms in an EPR $[10,11]$.

- The recommendations - should be based on consensus on evidence instead of consensus on opinions only.

- A sharable CIG - to share CIGs a guideline knowledge base should be reusable and the DSS should include a medical record query interface, terminology mediation, and an act interface[12].

- $\quad$ Access to the Electronic Patient Record (EPR) - makes the application of guidelines patient specific and therefore applicable at the moment of care.

Third, we looked from the perspective of a DSS [13]. Below we mention its characteristics.

- $\quad$ Retrieval of guidelines - when several potential guidelines exist, a guideline must be first retrieved from a guideline's repository and perhaps tested for its applicability prior to its application.

- Visualization of guidelines - to assist the care provider to understand the guideline better, the guideline encoded knowledge should be visualised in an unambiguous and intuitive manner.

- Integration with local clinical hosts - the DSS should be linked to other information 
systems (e.g., an order entry system), including the user interface, via standard messages and protocols.

- Persistent storage of guideline applications - to support intermittent application of complex GLs over long periods of time, the data gathered during an application of the GL (e.g., the user's decisions) must be stored in a persistent manner (e.g., database).

- Autonomous clinical control - the DSS must allow the clinician to override or ignore the GL's advice or data interpretation.

- Support for "look ahead" - to support queries about the meaning of recommended actions, the DSS should enable its users to explore dynamically what the guideline requires in each future situation without actually applying it (i.e., to carry out a "What If" query).

- Minimise user interaction - due to the short duration of clinical consultations, the DSS must not require too much user attention. For example, the system should indicate when sufficient data have been provided for the current decision step, without requesting an unnecessary data entry by the user.

- Capability to handle variable access to electronic patient data - the DSS should be able to profit from being connected to an EPR. It should be able to upgrade its level of support according to the functionality of the EPR; but it should also be able to apply the GL, even in the situation of not having optimal EPR-based support, or when EPR is not available at all.

- Capability to handle different levels of guideline representation - the DSS should be able to handle GLs specified at different levels of representation, ranging from structured text to a fully formal, machine-understandable format.

\section{RQ2: What should be the architecture of an intention-based decision support system?}

Guidelines implemented into a DSS are strictly defined and fixed. In situations where a patient's state or local constraints do not allow following the guideline exactly, a physician has to deviate from the guideline. As we noted, these deviations from the guideline may be still in the 'spirit' of the guideline, thus they should be acceptable.

A decision support system based on intentions should not only address 'what is the next action' but also 'why this is the next action'. Intentions can thus be used to explain the user the reason why the actions have to be carried out. This feature can be exploited to familiarise oneself with the guideline or for educational purposes.

An intention-based system should analyse the data about the actions of the physician to recognize the plan that he wants to execute, which plan then can be compared with the plan in the guideline. However, to abstract the motives and plans of a physician and compare them with the plans of the guideline is considerably difficult (Shahar et al. [14] Sips et al. [15]). 
A single data entry in the EPR can be directly compared with the "action to be performed according to the intention" formulated in the guideline, given that the used vocabularies are the same. It works as follows. The system will compare the name of the action entered into the EPR with the name of the action, which should be performed according to the guideline. If there is a match, the user works according to the guideline. If there is no match, the system will retrieve from the domain ontology all actions having the same purpose as the purpose of the action of the guideline. If one of those actions matches the physician's action, this action is accepted. In case of medications alternative medications can have a different titration protocol than the medication advised by the guideline. This can cause that the treatment plan of the alternative medication is different from the treatment plan followed in the guideline. In GASTINE therefore for each medication the respective titration protocols are stored. When the alternative medication is prescribed the corresponding titration protocol overwrites the titration protocol of the preferred medication in the guideline.

In consequence,

- the standardised terminology used in creating the guidelines e.g., medications, examination tests, laboratory tests, needs to have a defined attribute "purpose",

- a general ontology of intentions should be created that makes it possible to use the terms for intentions across different systems,

- $\quad$ every action can have multiple purposes,

- guidelines need to be implemented into computer systems using the same terminology,

- there need to be a ranking of alternatives with the same purpose, and there should be a possibility to make this ranking specific for gender, race, age, and local adaptation,

- designing intention-based guidelines using flowcharts, where intentions are executed in parallel or in sequence, lets us control the guideline flow and makes giving relevant advice easier,

- intentions can be decomposed into other intentions (into lower-level intentions) or they can directly refer to a plan of actions.

We developed the GASTINE system with the above mentioned characteristics. We realised that by designing an Intention Problem Solving Method (PSM). The PSM's template contains the problem solving roles, necessary for the PSM to function. For an Intention PSM these roles are pre-conditions, abort conditions (only used if all plans would fail) and goals. Similarly the plans (actions and post-intention actions) can be entered into the template (using other tabs). After entering these data, the Intention PSM is fully instantiated. Since the PSM consists of a number of decision steps, it can be executed in the same way as the remaining part of the guideline. An intention PSM is activated when 
its abort conditions are false and pre-conditions are true. The goal plays a role of decision step. If the goal is false (not yet reached), a sub-intention or action plan should follow. If the goal is true, the plan stored in the post-intention actions should be executed. The Dutch Heart Failure protocol was then implemented in GASTINE and we then verified the resulting DSS. Finally we validated the system using historic patient data.

\section{RQ3: - Do intentions actually provide more flexibility in making medical decisions?}

We evaluated whether the proposed architecture for a DSS using intentions actually provides the flexibility we envisaged. We understand by flexibility the capability of the system not to react in situations where the physician does not work according to the guideline but in its spirit.

There is a difference in giving advice in the pro-active and the re-active mode. In the pro-active mode the system does not have to check whether the advice is in the spirit of the guideline because the system's advice follows the guideline. However, in the reactive mode the system warns the care provider in case of incorrect actions. The system's flexibility therefore can only be studied in the re-active mode.

Since in the validation study it appeared that in only $24 \%$ of the patient encounters the system's advice matched exactly the actual treatment given, the re-active mode produced many alerts and additional advices. In the phase of evaluating the system we allowed all alerts, warnings, and advices to appear, even if the actions taken were in the spirit of the guideline (e.g., prescribing an alternative medication). For actions that are in the spirit of the guideline, the system can be tuned not to show warnings immediately. The system can be tuned to generate a warning only when the alternative medication has been prescribed, for example, (a) 5 times, or (b) after a certain period of time, or (c) when a predefined value is reached by some parameter in the EPR. Not showing warnings at all as well as not showing alerts, we agreed, is not an option. The flexibility that the system gives is desired but the security of following the guideline is required as well.

When our system recognises that the alternative medication has the same purpose as the medication suggested by the guideline it allows the user to proceed without exactly following the guideline. However, when an alternative medication was used and the system did not contain the titration protocol for this medication, it could not present more detailed information about the dosage.

It could be concluded that GASTINE had the flexibility to accept actions taken by the physician that were in the spirit of the guideline. 


\section{RQ4 - Do intentions help to make changes and updates in a DSS easier?}

Intentions lead to flowcharts that are structured in a more insightful way. In that sense the flowcharts are less complex. Each flowchart is devoted to a certain intention and therefore better to understand and follow.

The complexity of the implemented guideline of course also depends on the complexity of the original guideline and on the experience of the implementer. It is our experience after years of working with computer based systems such as GASTON and GASTINE that various implementers build flowcharts using primitives in different ways. The more experienced the implementer, the less complex the solution and the more efficiently the system features are used.

Intentions can make updates of the DSS easier as flowcharts regarding the same intention are located together and often use the same functions.

Also, systems like GASTON and GASTINE allow re-use of the flowcharts in different parts of the guideline. That feature, next to intentions, also makes the updating of guidelines easier. As a consequence, making changes and updates is easier, because changes in such a flowchart have to be made only once, independent of where this flowchart is re-used. In case new medications have to be included in the treatment protocol there are two steps that should be carried out. First, when the purpose of such a medication is known, it can be easily added to the medication ontology and after that the system will immediately generate general advice e.g., "Patient receives diuretics which are not mentioned in the protocol. The patient still has oedema, please increase the dose of diuretics if possible". Second, by placing the titration procedure of this medication in the right flow, the DSS is enriched with information about an alternative medication so that the system from then on will also accept this medication as an action in the spirit of the guideline if the purpose of the medication is the correct one and the dose is according to its titration procedure. The changes have to be made once in the flowcharts related to the purpose of this medication. If the flowchart is re-used, those places in the guideline will be updated automatically.

Because of the more transparent structure of the implemented guideline an exhaustive verification of the guideline was possible. This would not have been the case when the guideline was formalised and implemented without the use of intentions: the number of checks is then much too large. As a result, intentions make not only the maintenance of guidelines easier but also their verification.

\section{RQ5 - Do intentions help to reduce the complexity of flowcharts and thinking?}

The answer to the question whether guideline formalization using intentions is less complex is subjective. As explained above, the complexity is reduced resulting in an easier maintenance of the guideline. The flowcharts can be easier understood because of 
the use of intentions. Also intentions are used to explain why certain recommendations were given.

But, the use of intentions requires a change in the way of thinking of the implementer. In pro-active mode the implementer just follows the guideline's steps whereas in the re-active mode the implementer has to determine which action was carried out. What is the purpose of this action? To what intention does it belong? Is it according to the guideline? If not, is it acceptable because the action is in the spirit of the guideline? These considerations have to be translated with the help of the guideline's building blocks.

It can be concluded that by using intentions it is easier to arrange treatments according to their purposes. That makes the overview of possible alternatives less complicated and creates an opportunity to arrange those options in a preference order. On the other hand different flowcharts are needed for the pro- and re-active mode which makes updating the flowchart more complex.

Because of the use of intentions the users can be explained via the backtracking information why a certain action is recommended. The intentions indicate the reason why actions have to be carried out. With the original guideline only previous steps can be displayed but these steps only concern previous actions and the reason why these actions were carried out does not have to be apparent.

\subsection{Strengths and Weaknesses of our Approach}

In our approach to the development of an Intention-based Decision Support System we arrived at a solution that is as much as possible generally applicable and implementable. Therefore, it is applicable to a broad range of clinical practice guidelines, rather than being a single system solution. The Intention-based approach is general provided that the terminology and the ontology regarding intentions used across different systems is standardised.

To investigate whether the new features of GASTINE make a difference in building and executing computerised guidelines, we started to prove that building guidelines using intentions is possible. Structuring the guideline according to the different intentions leads to a number of sub-flowcharts that contain a limited set of conditions. So, during verification tests we did not have to test all value combinations of all variables simultaneously, which reduced the number of combinations to be tested considerably. Because the code of the GASTINE primitives was correct, during verification tests we did not have to use all combinations as required by the Multiple Condition Coverage (MCC) method. This reduced the number of combinations even further. We could test GASTINE exhaustively. The MCC method is time consuming, but in a period of seven weeks all the 
relevant flowcharts were checked and where needed corrected.

Further, during the validation tests we found out that in $99 \%$ of the cases the system gave an understandable explanation of how it did proceed to generate an advice. In $94 \%$ of the cases the tester agreed that advice with explanation was preferred over plain advice. Consequently, the backtracking information did provide users more insight into why recommendations were given. The backtracking information is clustered per (sub) intention which means that all actions related to one intention are presented together. Such a feedback makes it easier to understand the steps that led to the advice.

When a physician deviates from the guideline he is given a warning and receives advice according to the guideline. However, he may still decide to continue his course of action. All steps taken are saved in the Evaluation Steps and Evaluation Response tables and can be used to detect insufficiencies in the guideline (guideline is perhaps outdated) or in the physician's medical practice (lack of awareness of an existing different treatment process). Unfortunately our system has also a few weaknesses. During the formalization and implementation of the guideline errors occurred. However, they were the usual ones: (1) errors made in the criteria logic during the creation of the flowchart; (2) errors due to incompleteness of the protocol; (3) a programming problem.

To create a formalised guideline for use in the re-active mode requires a different way of thinking of the implementer. The original guideline cannot directly serve as a template for the formalised guideline.

We tested the system using historic patient data. However, our results are based on only 7 patients with in total 70 encounters about which not always all data were available. Still, we believe that our findings regarding flexibility, insightfulness, and usefulness of our system will also be valid when a larger number of patients and encounters is used. In our study we noted that in 9 encounters the NYHA class was not mentioned. In case of missing data, the suggestion of the tester was that it is better to give no advice about the treatment at all, since an advice could mislead the health professional. However, a warning about missing data can be given that offers the possibility to fill in these missing data directly. After data entry the advice of the system can be generated using the enriched data set. In this way GASTINE will increase the completeness of the data.

Further, during validations tests we found that deviations from the guideline occurred consistently. The system could have prevented deviations from the guideline in almost $50 \%$ of the encounters. However, the value of the system still needs to be tested in practice.

The tester was not fully satisfied with the backtracking information. The system provides the final result of the assessment of a decision step, but does not mention the outcome of intermediate steps. This makes the interpretation of the final outcome of a decision step more difficult. The enrichment of the backtracking information with the results of these 
intermediate steps would help the user to understand better why a particular advice was given or why his actions generated a warning. The system can be changed easily to accommodate this feature.

There were a number of situations where the tester indicated that the system gave incorrect advice. But, does this make the system less useful? This question leads to two follow-up questions. (a) Are the deviations due to errors in the formalisation of the guideline and therefore correctable? or (b) Are the errors due to the guideline itself? We conjectured and partly observed that the deviations were due to (1) the interpretation of what is a (too) high potassium value, (2) the lack of an additional criterion in the guideline concerning the presence of oedema and (3) the lack of a formalised concept of a "stable patient". So, may we thus conclude that the guideline was not correct or not sufficiently concrete for these situations and that it does not use the concept of stable patient? No, we need to remember that in the guideline we find general descriptions of which treatment should be given under which circumstances (e.g., "By all patients with heart failure the ACE inhibitor treatment in combination with diuretics should be considered"). In the (local) protocol we find detailed criteria that are used to determine how the treatment should be given. The more detailed the information stored in the protocol and/or our system is (e.g., criteria, alternatives), the better the advice and critique. However, the specification of these detailed criteria also leads to one of the errors mentioned above: a too strictly defined boundary between normal and high. The other two errors were due to missing specifications.

In conclusion, our research indicated that the use of intentions makes the use of the system more flexible and reduces the complexity of the implemented guidelines. This allows the system to explain the reasons why warnings are given or to explain the reasons behind the advice. Also intentions make the system easier to maintain.

\subsection{Related Research}

The research presented in this thesis was inspired by studies from other domains of medical informatics and computer science.

In Chapter 2 we presented a literature overview on Clinical Practice Guidelines, Computer Interpretable Guidelines, and Decision Support Systems. We showed that many research groups all over the world are trying to support the healthcare system in realising its biggest challenge: providing the best healthcare (process) of the highest quality (outcome) at the lowest costs.

The obstacles and shortcomings that one group is facing, may become a source of inspiration for other groups. 
In this context the way a hungry cat finds food as described by Winikoff et al. [16] inspired us with ideas of how to change the system - GASTON - to make the Decision Support System more flexible. We presented his example in more detail in the chapter 1. When circumstances change and the cat's plan cannot be accomplished, he goes for an alternative set of actions, but still with the purpose to reach his goal, getting food. He needs to change his primary plan to fulfil his intention. Similarly, a physician may select an other treatment than specified in the guideline, because of a patient specific situation or a local or temporary constraint, not foreseen when the guideline was developed.

There are other groups who implemented intention-based guidelines. In their work, Miksch et al. [17] and Shahar et al. [18] introduced intentions in ASBRU. They define intentions as high-level goals that support special tasks such as critiquing and modification. Intentions are patterns of states or actions to be achieved, maintained, or avoided, during or after the execution of the plan [19]. ASBRU represents clinical guidelines as skeletal plans. Skeletal plans are plan schemata at various levels of detail, capturing the essence of the procedure, but leaving room for execution-time flexibility in the achievement of particular goals. The ASBRU syntax is quite elaborate. Current execution engines for ASBRU only use part of the syntax. To our knowledge intentions in the way we defined them and made them explicit cannot be processed by these engines.

Further, Marcos et al. [19] discussed the critiquing mechanism in Asbru. They concluded that it is difficult to match expert actions with steps in the protocol. Sips et al. [15] used low-level intentions (context independent intentions) to characterise both actions recorded in the EPR and actions in the guideline. They could match $70 \%$ of the EPR action sequences with guideline execution sequences. We also believe that using the Purposes (the equivalent of low-level intentions) of every action is a crucial element of plan recognition and implemented it in our system. By using the purpose of an action makes it possible to analyse intentions of the physician without abstracting them from multiple EPR entries.

\subsection{Significance of the Research}

By introducing intentions into guidelines we have made it possible to accept, at least for the first number of encounters, actions that are in the spirit of the guideline. In the case of medications this means that not only alternative medications can be accepted but also that the system is able to use titration protocols differing from the titration protocol of the medication used in the guideline. Only when the physician does not follow the titration protocol the system is not able to determine that the physician acts in the spirit of the guideline. So our approach increases the flexibility of the DSS. The system does not 
have to infer the treatment plan of the physician from data in the EPR.

In this way we made the system more acceptable to physicians. Also, by providing backtracking information the physician better understands why advice or warnings are given. We showed as well that because of the use of intentions the guideline could be exhaustively verified which would not have been possible without the use of intentions.

\subsection{Further Work}

The emphasis of the research has been on the design and development of a framework using Intentions in building computer-interpretable guidelines. Further work may involve (1) the development of an intention ontology, (2) the formalisation and implementation of more guidelines needing the use of intentions, and (3) testing the system in practice on a bigger scale.

Further development of an intention ontology. In their study Fox et al. [20, 21] summarised the current state of art for modelling clinical goals and compared different approaches. The authors conclude that there is promising convergence but further work is needed to come to a final scheme.

Implementation of more guidelines. Further translation, formalisation, and implementation of guidelines into systems like GASTINE may produce new requirements not considered by us. It also may further confirm the suitability of our approach.

Further using the system prospectively in real time encounters will be interesting to validate whether GASTINE performs as well on a larger group of patients as on historical cases. Additionally, it is necessary to evaluate the use of the DSS in the daily workflow of healthcare providers. Also further research should focus on how to cope in decision steps with measurements that fall near the boundaries of ranges.

\subsection{Concluding Remarks}

Our main goal was to develop a system that can communicate with the physician in a flexible way (through advice and alerts), allowing him to take a path that may not be completely according to the guideline, but within its spirit. This provides the physician with the option to make decisions based on his previous experience, on local agreements, or the patient's preferences, which is a very important issue.

The guideline representation that we discussed here supports the (1) design of intentionbased guidelines, (2) their execution, (3) reasoning about intentions during runtime, and (4) providing flexible feedback. 
Intention-based Decision Support Systems can be beneficial for health care in many ways. We believe that they can improve a physician's adherence to guidelines because the guidelines (1) support and do not disturb the workflow; (2) make adaptation to local standards easier; (3) can make their evaluation simpler and faster. The system implements the original guideline and all local modifications to the guideline are stored separately as alternative actions.

We envision that implementing intention-based guidelines might help in evaluating whether the guidelines are up-to-date. By using an explicit representation of the intentions behind guideline actions and guideline steps, a flexible decision support system can be built that does not only check the adherence to the formal guideline but also evaluates clinical activities in the light of the intention of the guideline. If some actions in the guideline are only recommended by a few physicians to their patients, we may conclude that those actions might not be current anymore or that users might not be aware of some new possibilities and may need extra information or training.

Based on the validation tests we tentatively may conclude that the system will be useful in practice. However, only when we test the system's use in practice will we be sure that the system indeed reduces deviations from the guideline.

Finally, we would like to underline the importance of the physician and computer working together. When the healthcare provider does something different from what the guideline suggests the system gives a warning and does not accept the physician's actions, preventing him from making a mistake. The computer system collects data, checks criteria, and executes rules faster than humans, but it provides advice based on pure facts; these pure facts are for the system the starting point for determining a treatment. In contrast to the computer system, the healthcare provider can communicate with the patient, can assess his emotional state, can respond to his needs, and can take responsibility for the performed actions. The two 'actors' combined create a very powerful tool that can improve quality of care and patient outcomes. But this is only possible when the DSS allows the physician to deviate from its recommendations. This becomes possible when intentions are used in the guideline. 


\section{References}

1 Peleg M, Tu SW. Design patterns for clinical guidelines. Artif Intell Med. 2009 Sep;47(1):1-24.

2 Leslie SJ, Hartswood M, Meurig C, McKee SP, Slack R, Procter R, et al. Clinical decision support software for management of chronic heart failure: development and evaluation. Comput Biol Med. 2006 May;36(5):495-506.

3 Goldstein MK, Hoffman BB, Coleman RW, Tu SW, Shankar RD, O'Connor M, et al. Patient safety in guideline-based decision support for hypertension management: ATHENA DSS. Proc AMIA Symp. 2001:214-218.

4 Vogelzang M, Zijlstra F, Nijsten MW. Design and implementation of GRIP: a computerised glucose control system at a surgical intensive care unit. BMC Med Inform Decis Mak. 2005;5:38.

5 Shaneyfelt TM, Mayo-Smith MF, Rothwangl J. Are guidelines following guidelines? The methodological quality of clinical practice guidelines in the peer-reviewed medical literature. JAMA. 1999 May 26;281(20):1900-1905.

6 Littlejohns P, Cluzeau F. Guidelines for evaluation. Fam Pract. 2000 Feb;17 Suppl 1:S3-6.

7 Burgers JS, Cluzeau FA, Hanna SE, Hunt C, Grol R. Characteristics of high-quality guidelines: evaluation of 86 clinical guidelines developed in ten European countries and Canada. Int J Technol Assess Healthcare. 2003 Winter;19(1):148-157.

8 Shiffman RN, Dixon J, Brandt C, Essaihi A, Hsiao A, Michel G, et al. The Guideline Implementability Appraisal (GLIA): development of an instrument to identify obstacles to guideline implementation. BMC Med Inform Decis Mak. 2005;5:23.

9 Field M, Lohr K, (Eds). Guidelines for clinical practice: from development to use. Institute of Medicine, Washington, DC: National Academy Press. 1992.

10 Patel VL, Branch T, Wang D, Peleg M, Boxwala A. Analysis of the process of encoding guidelines: a comparison of GLIF2 and GLIF3. Methods Inf Med. 2002;41(2):105-113.

11 Wang D, Peleg M, Tu SW, Boxwala AA, Ogunyemi O, Zeng Q, et al. Design and implementation of the GLIF3 guideline execution engine. J Biomed Inform. 2004 Oct;37(5):305-318.

12 Tu SW, Campbell JR, Glasgow J, Nyman MA, McClure R, McClay J, et al. The SAGE Guideline Model: achievements and overview. J Am Med Inform Assoc. 2007 Sep-Oct;14(5):589-598.

13 Young O, Shahar Y, Liel Y, Lunenfeld E, Bar G, Shalom E, et al. Runtime application of HybridAsbru clinical guidelines. J Biomed Inform. 2007 Oct;40(5):507-526.

14 Shahar Y, Miksch S, Johnson P. A Task-Specific Ontology for the Application and Critiquing of Time-Oriented Clinical Guidelines. Lecture Notes In Computer Science Proceedings of the 6th Conference on Artificial Intelligence in Medicine in Europe. 1997;1211:51-61.

15 Sips RJ, Braun L, Roos N. Enabling protocol-based medical critiquing. Stud Health Technol Inform. 2006;124:471-476.

16 Winikoff M, Padgham L, Harland J, Thangarajah J. Declarative and Procedural Goals in Intelligent Agent Systems. 2002 Toulouse, France, April 22-25, 2002. 
17 Miksch S, Shahar Y, Johnson P. Asbru: A task-specific, intention-based, and time-oriented language for representing skeletal plans. Proceedings of the Seventh Workshop on Knowledge Engineering Methods and Languages. 1997((Milton Keynes, UK, 1997)):9-1 - 9-20.

18 Shahar Y. The Asgaard project: a task-specific framework for the application and critiquing of time-oriented clinical guidelines Artificial Intelligence in Medicine. 1998;14(1):29-51(23).

19 Marcos M, Berger G, van Harmelen F, ten Teije A, Roomans H, Miksch S. Using critiquing for improving medical protocols: harder than it seems. In Proc of the 8th European Conference on Artificial Intelligence in Medicine (AIME-2001). 2001:431-441.

20 Fox J, Alabassi A, Black E, Hurt C, Rose T. Modelling clinical goals: a corpus of examples and a tentative ontology. Stud Health Technol Inform. 2004;101:31-45.

21 Fox J, Alabassi A, Patkar V, Rose T, Black E. An ontological approach to modelling tasks and goals. Comput Biol Med. 2006 Jul-Aug;36(7-8):837-856. 
Summary 
Healthcare is a domain with many different fields and specializations. Currently, we observe several changes that take place in that domain. Four important ones are: (1) paper patient records are being replaced by electronic patient records (EPR); (2) workflows and treatments are supported by computers; (3) quality issues have become a major topic: physicians increasingly work in an evidence-based way; (4) the growth of medical knowledge is so fast that most physicians are not able to keep up with this development and have to be supported by knowledge-based systems. With these changes, new challenges and obstacles appear.

One of the greatest challenges of this moment is the way healthcare is struggling with the dilemma of providing the best healthcare (process) of high quality (outcome) at the same or lower costs. Clinical practice guidelines (CPGs) were developed to support physicians but such paper-based guidelines are quite time consuming to go through and unsuitable for just-in-time use at the point of care. Moreover their quality was not always optimal.

One of the disciplines that try to help healthcare to find a balance between those requirements is medical informatics. As active participants in this field, medical informaticians are interested in supporting healthcare with services based on Information and Communication Technologies (ICT).

To adapt the clinicians' behaviour, during patient encounters CPGs should provide adequate patient-specific decision support, which is reliable and understandable by the clinicians. That is why guidelines are implemented in a Computer Interpretable Guideline (CIG) formalism that provides support based on patient data. Overtime, we observed an increase of guidelines implemented in Decision Support Systems (DSSs) that help users to follow the guideline. In a DSS CIGs are implemented in a formalised way. The DSS retrieves all necessary patient data from an EPR and generates patient specific advice based on these patient data and the guideline content.

However, DSSs are not sufficiently flexible. For instance, even when the clinician acts in the spirit of the guideline DSSs directly issue a warning. When clinical research leads to new insights or new pharmaceuticals become available, this knowledge should be implemented in the system, preferably directly and easily. Therefore, the system should be able to adopt new insights in a procedural way. Also, the guideline must be implemented in a particular clinical setting. Ultimately, all clinical decisions are made in a local environment, which includes the practice culture, the characteristics of the particular clinician, and the characteristics of the particular patient. It seems paradoxical to produce practice guidelines to reduce variations in practice and then integrate mechanisms that facilitate local variations. However, it seems to be necessary in order to achieve clinician acceptance.

The main contribution of the research presented in this thesis is the introduction of Intentions and their use in formalizing guidelines and their implementation into computer 
systems. Formalization of guidelines means the translation of narrative (paper-based) guidelines into $\mathrm{CIGs}$ by the use of computer interpretable templates. An intention is a determination to act in a certain way; it is a high level goal description which is abstract and not quantified (e.g., lowering blood pressure).

In Chapter 1 we present historical information about the development of guidelines and Decision Support Systems in the field of health care. We briefly discuss relevant definitions and introduce computer interpretable guidelines. We also present the GASTON framework that was designed and developed at the Medical Informatics department at Maastricht University and the Signal Processing Systems division of the Department of Electrical Engineering of the Eindhoven University of Technology in the Netherlands. GASTON is a tool for representing and executing computerised clinical guidelines. The problem to be solved by the research discussed in this thesis is: What features must a DSS (or the CIG) possess to be more flexible and allow actions in the spirit of the guidelines in order to encompass fully satisfyingly the intentions behind the actions?

This led to the following five main research questions.

$1 \quad$ What are high quality guidelines and what is the best way to create them?

2 What should be the architecture of an intention-based decision support system?

3 Do intentions actually provide more flexibility in making medical decisions?

$4 \quad$ Do intentions help to make changes and updates in a DSS easier?

5 Do intentions help to reduce the complexity of flowcharts and thinking?

In Chapter 2 we present the results of a literature review to obtain insight into factors that influence the design and implementation of guidelines. An extensive search of the scientific literature was carried out focused on guideline characteristics, guideline development and implementation, and guideline dissemination. We present studies that enabled us to explain the characteristics of high quality guidelines, and new advanced methods for guideline formalization, computerization, and implementation. We show how the guidelines affect processes of care and patient outcome. We discuss the reasons of low guideline adherence as presented in the literature.

In Chapter 3 we describe the development of a framework for clinical practice guidelines that not only allows the representation of best practices, but also facilitates reasoning about acceptable alternatives for those best practices. We present the design of a representation formalism for intentions of guidelines and guideline steps. We called our system GASTINE (GASTon INtentional Expressions). The developed formalism was used to represent a heart failure clinical guideline. It is demonstrated that the representation of intentions provides the needed flexibility to avoid unnecessary errors and warnings. 
In Chapter 4 we focus on the evaluation of the design of the framework for computerised intention-based clinical practice guidelines and on the implementation of the runtime features such as plan recognition and backtracking. We present the current implementation of intention-based expressions in GASTINE and analyse some generic shortcomings. We explain how these shortcomings were addressed. We also explain how plan recognition and backtracking work and how they improve the system.

In Chapter 5 we describe the first evaluation phase, verification. We looked for an answer to the question whether we built the system right. We verified whether our system was well-engineered and error-free. We show the results of those verification tests using the formalised and implemented Dutch national heart failure guideline. A simple EPR with a database created in Office Access was created to support the verification and validation tests. We explain the Multiple Condition Coverage (MCC) method that we used during verification of our system. This method requires that all value combinations of the variables present in the conditions of a decision step are tested. However, because the code of the primitives carrying out the logical operations have been tested many times the number of value combinations could be reduced. Also, since intentions divide a flowchart in several sub-flowcharts the number of value combinations to be tested is reduced considerably. Several errors in the guideline representation were detected and corrected.

In Chapter 6 we show the results of the second evaluation phase, validation. Now the question whether we built the right system had to be answered. In other words, is the system useful? We had to prove that the intentions underlying the actions allow more flexible advice, lead to less unnecessary warnings, and provide more insight into the reasons behind the generated advice. To validate the system we used the data of 7 patients with 70 patient-physician encounters in total. Validation was carried out in two phases. In phase one, for each patient-physician encounter, two types of data were entered into the Electronic Patient Record system interfaced to GASTINE: (1) laboratory and physical examination results and (2) the medication history of the patient. Based on the entered data the DSS generated advice. The system was tested in both the proactive and the re-active mode. In the pro-active mode the system generated advice based on historical data; in the re-active mode the system determined whether the prescribed medication was according to or in the spirit of the guideline, and if that was not the case, generated warnings. Those advices and warnings were stored in a specifically created database. In phase two, a cardiologist together with a heart failure nurse practitioner appraised and compared the treatment advice generated by the system with the original treatment. In doing so they made use of backtracking information. 
During the validation tests we found out that in $99 \%$ of the cases the system gave an understandable explanation of how it did proceed to generate an advice. In $94 \%$ of the cases the tester agreed that advice with explanation was preferred over plain advice. Consequently, the backtracking information did provide users more insight into why recommendations were given. Further, we also found that deviations from the guideline occurred consistently in daily practice. The system could have prevented deviations from the guideline in almost $50 \%$ of the encounters. However, the value of the system still needs to be tested in practice.

In Chapter 7 we describe our principle findings, and address the strengths and weaknesses of our approach. We relate our work to that of others and provide an outlook on future research.

Our main focus was to develop a system that can communicate with the physician in a flexible way (through advice and alerts), allowing him to take a path that may not be completely according to the guideline, but within its spirit. This provides the physician with the option to make decisions based on his previous experience, on local agreements, or on the patient's preferences, which is a very important issue.

The most important conclusions that can be formulated based on the research presented in this thesis is that Intention based Decision Support Systems can be beneficial for health care in many ways. We believe that they can improve a physician's adherence to the guidelines because (1) the guidelines support and do not disturb the workflow; (2) they make adaptation of the guidelines to local standards easier; (3) they can make the evaluation of the guideline simpler and faster; and (4) the system implements the original guideline and stores all local modifications to the guideline separately as alternative actions.

Finally, we would like to underline the importance of the physician and computer working together. The healthcare provider might do something different from what the guideline suggests or patient data allows; in that case the system gives a warning and does not accept the physician's actions, preventing him from making a mistake. However, actions in the spirit of the guideline are accepted. The computer system collects data, checks criteria, and executes rules faster than humans, but it provides advice based on dry facts; this information is for the system the starting point for the treatment. In contrast, the healthcare provider is the one who can communicate with the patient, can assess his emotional state, can respond to his needs and take responsibility for the performed actions. Those two approaches combined create a very powerful tool that can improve quality of care and patient outcomes. And the physician stays in charge and remains responsible. 
Samenvatting 
Artsen en verpleegkundigen worden momenteel geconfronteerd met verschillende veranderingen in de gezondheidszorg zoals: (1) de introductie van het elektronisch patiënten dossier (EPD); (2) ondersteuning door computers van werkstromen en behandelingen; (3) kwaliteitsborging: artsen en verpleegkundigen voeren steeds meer handelingen uit, die gebaseerd zijn op de best beschikbare informatie over doelmatigheid en doeltreffendheid; en (4) de medische kennis neemt zo snel toe, dat de meeste artsen de ontwikkelingen niet meer bij kunnen houden en ondersteund moeten worden door op kennis gebaseerde systemen. Deze veranderingen vormen een uitdaging maar zijn anderzijds ook struikelblokken. Eén van de grootste uitdagingen is een oplossing te vinden voor de opgave om de beste gezondheidszorg (proces) van de hoogste kwaliteit (uitkomst) tegen gelijke of lagere kosten te leveren. Klinische richtlijnen kunnen artsen en verpleegkundigen daarbij ondersteunen. Papieren richtlijnen zijn tijdens het bezoek aan de arts of aan het ziekbed niet gemakkelijk te gebruiken. Het vinden van de juiste behandeling is tijdrovend terwijl de kwaliteit van de richtlijnen niet altijd optimaal is. Het vakgebied Medische Informatica onderzoekt middelen, vaak gebaseerd op de toepassing van informatiesystemen, die dit probleem kunnen verminderen dan wel oplossen.

Om het handelen van clinici te ondersteunen moet een systeem de arts op de patiënt gerichte betrouwbare en inzichtelijke adviezen bieden. Een beslissingsondersteunend systeem (Decision Support System, DSS) dat gebruik maakt van richtlijnen kan, in tegenstelling tot de papieren richtlijn, door gebruik te maken van patiëntengegevens uit een EPD en op basis van informatie uit een computer interpreteerbare richtlijn (Computer Interpretable Guideline, CIG) patiënt specifieke adviezen leveren.

De afgelopen tijd zijn er meerdere DSS-en ontwikkeld. Veel DSS-en zijn echter niet voldoende flexibel. Wanneer de clinicus bijvoorbeeld in de geest van de richtlijn handelt, zal het DSS toch een waarschuwing geven, wat de acceptatie van een DSS door de clinicus negatief kan beïnvloeden, omdat dergelijke onnodige waarschuwingen verstorend kunnen werken. Als door klinisch onderzoek of door de introductie van nieuwe geneesmiddelen betere behandelmogelijkheden ontstaan, moeten deze in het systeem op een eenvoudige wijze opgenomen kunnen worden. Bovendien wordt een richtlijn altijd in een bepaalde setting geïmplementeerd. Deze setting wordt bepaald door een eigen cultuur en door de kenmerken van behandelaar en patiënt. Het lijkt paradoxaal om richtlijnen te ontwikkelen met het doel onnodige praktijkvariatie te voorkomen en dan toch mogelijkheden te scheppen om lokale variaties te faciliteren. Echter, om acceptatie door clinici te bewerkstelligen blijkt dit nodig te zijn.

De belangrijkste bijdrage van het onderzoek, beschreven in dit proefschrift, is de invoering van intenties en hun gebruik ten behoeve van het formaliseren van richtlijnen en hun implementatie in informatiesystemen. Het formaliseren van richtlijnen houdt in dat de vrije tekst uit een papieren richtlijn wordt vertaald in een vorm, die de computer kan 
interpreteren. Onder intentie verstaan we het vaste voornemen om op een bepaalde manier te handelen; het is een abstracte en niet gekwantificeerde beschrijving van het doel dat men wil bereiken (zoals 'verlagen van de bloeddruk').

In hoofdstuk 1 worden de ontwikkelingen met betrekking tot het gebruik van richtlijnen binnen het domein van de gezondheidszorg in de afgelopen 30 jaar gepresenteerd. De verschillende definities van termen in gebruik bij richtlijnen en DSS-en passeren de revue. Tevens introduceren we GASTON, het systeem dat ontwikkeld werd binnen de voormalige afdeling Medische Informatica van de Universiteit Maastricht en de Signal Processing Systems groep van de Faculteit Electrical Engineering van de Technische Universiteit in Eindhoven. GASTON is een tool voor het representeren en uitvoeren van CIGs.

De vraag die het onderzoek, beschreven in dit proefschrift, moet beantwoorden is: Welke kenmerken moet een DSS (of een CIG) hebben om meer flexibel te zijn zodat handelingen kunnen worden geaccepteerd die in de geest van de richtlijn zijn en voldoen aan de intenties achter deze handelingen? Dit leidde tot de volgende vijf onderzoeksvragen.

1 Wat zijn richtlijnen van hoge kwaliteit en wat is de beste manier om ze op te stellen?

2 Hoe dient de architectuur van een op intenties gebaseerd beslissingsondersteunend systeem eruit te zien?

3 Staan intenties inderdaad meer flexibiliteit toe bij het nemen van medische beslissingen?

4 Wordt het wijzigingen van een DSS door het gebruik van intenties gemakkelijker?

5 Reduceren intenties de complexiteit van stroomdiagrammen en de manier van denken?

In hoofdstuk 2 worden de resultaten van een uitgebreide literatuurstudie gepresenteerd die werd uitgevoerd om inzicht te verkrijgen in factoren die het ontwerp en de implementatie van richtlijnen beïnvloeden. De studie concentreerde zich op richtlijn karakteristieken, de richtlijn ontwikkeling en implementatie en op de verspreiding van richtlijnen. We bespreken hoe richtlijnen zorgprocessen en de uitkomst van de patiëntenbehandeling beïnvloeden. De in de literatuur beschreven redenen waarom van richtlijnen wordt afgeweken worden door ons bediscussieerd en becommentarieerd.

Hoofdstuk 3 beschrijft de ontwikkeling van een raamwerk voor de ontwikkeling van CIGs, waarmee niet alleen op ' best practice' gebaseerde beslissingen maar ook alternatieven voor deze 'best practice' beslissingen worden ondersteund. Het ontwerp van een representatie formalisme voor intenties en hun implementatie in richtlijnen wordt gepresenteerd. Het systeem wordt GASTINE (GASTon INtentional Expressions) genoemd. Het formalisme is gebruikt om de hartfalen richtlijn te representeren. Aangetoond wordt dat het gebruik van intenties de benodigde flexibiliteit biedt die nodig is om onnodige foutmeldingen en waarschuwingen te vermijden. 
In hoofdstuk 4 wordt beschreven hoe de nationale richtlijn hartfalen met behulp van intenties geïmplementeerd werd in GASTINE. Tevens wordt besproken welke tekortkomingen bij een eerste evaluatie werden geconstateerd en wordt aangegeven hoe deze zijn verholpen. Verder wordt uitvoerig besproken hoe het doel van de door de arts voorgenomen acties wordt herkend en hoe de stappen, die hebben geleid tot het huidige advies, gebruikt kunnen worden om het systeem te verbeteren.

In hoofdstuk 5 wordt de verificatie van het systeem beschreven. Hierbij wordt antwoord gegeven op de vraag of het systeem correct ontworpen is.

Met behulp van een eenvoudig eigen ontwerp EPD met Access database is de implementatie van de Nederlandse hartfalen richtlijn geverifieerd. Met behulp van Multiple Condition Coverage (MCC) zijn alle mogelijke waardecombinaties van alle variabelen, die aanwezig zijn in de condities van de beslissingsstappen, getest. Doordat de code van de primitieven, die logische operaties uitvoeren, vele malen zijn getest kon het aantal waardecombinaties worden gereduceerd. Door het gebruik van intenties wordt het stroomdiagram opgesplitst in een aantal subdiagrammen. Hierdoor wordt het aantal waardecombinaties dat getest moet worden aanzienlijk verkleind. Het bleek daardoor mogelijk de implementatie van de richtlijn uitputtend te testen. De gevonden fouten konden alle worden hersteld.

Hoofdstuk 6 beschrijft de validatie van GASTINE. Hierbij wordt antwoord gegeven op de vraag of wij het juiste systeem hebben ontworpen. Voldoet het systeem wel aan de behoeften van de gebruiker? We moesten bewijzen dat de intenties inderdaad een meer flexibele advisering mogelijk maken, leiden tot minder onnodige waarschuwingen en meer inzicht bieden in de redenen achter het gegeven advies. Aan de hand van de data van 7 patiënten met een totaal van 70 patiëntencontacten is het systeem gevalideerd. De validatie werd in twee fasen uitgevoerd. In fase 1 zijn voor elk patiëntencontact relevante gegevens (laboratoriumuitslagen, resultaten van lichamelijk onderzoek en medicatiegegevens) in het EPD (dat gekoppeld is aan GASTINE) ingevoerd. Op basis van deze data genereert GASTINE een advies.

Zowel de proactieve als de reactieve wijze van werken van het systeem is getest. Proactief betekent dat het systeem op basis van historische data een advies genereert voordat de arts daadwerkelijk een actie onderneemt. Reactief betekent dat het systeem waarschuwt als de door de arts ingevoerde actie niet volgens of in de geest van de richtlijn is. In fase 2 vergeleken een cardioloog en een nurse practitioner hartfalen de werkelijk uitgevoerde stappen in het behandelplan met de door GASTINE op basis van de richtlijn voorgestelde stappen, daarbij gebruikmakend van de backtracking informatie.

Bij de validatie werd vastgesteld dat het systeem inzichtelijk maakt op grond waarvan een 
advies wordt gegeven. Het team dat de adviezen van het systeem vergeleek met de in de praktijk gegeven behandeling gaf aan, dat in $94 \%$ van de gevallen een advies met uitleg te prefereren was boven het advies alleen. De backtracking informatie leverde dus meer inzicht in het waarom van het advies. In de praktijk blijken afwijkingen van de richtlijn vaak voor te komen. Het systeem zou afwijkingen van de richtlijn kunnen voorkomen in bijna $50 \%$ van de patiëntencontacten. De waarde van het systeem moet echter nog wel in de praktijk getest worden.

Hoofdstuk 7 geeft een overzicht van de belangrijkste bevindingen uit het onderzoek en plaatst deze in de context van eerder onderzoek. Bovendien worden de sterke en zwakke punten van de gebruikte aanpak besproken en worden richtingen voor verder onderzoek aangegeven.

Ons belangrijkste doel was een systeem te ontwikkelen dat de arts op een flexibele wijze (via advies en waarschuwingen) kan ondersteunen door hem toe te staan af te wijken van de richtlijn, zolang maar wordt gewerkt in de geest van de richtlijn. Hierdoor heeft de arts de mogelijkheid te beslissen op basis van opgedane ervaring, lokale afspraken of de voorkeur van de patiënt.

De belangrijkste conclusie van het in dit proefschrift beschreven onderzoek is dat op intenties gebaseerde beslissingsondersteunende systemen op verschillende manieren een positieve bijdrage aan de gezondheidszorg kunnen leveren. Wij geloven dat een dergelijk systeem de kans op afwijken van de richtlijn verkleint omdat (1) de richtlijn het werkproces ondersteunt en het werkproces niet verstoort door het geven van onnodige waarschuwingen; (2) de intenties aanpassing aan lokale standaarden vereenvoudigen; (3) intenties de evaluatie van richtlijnen versnelt en versimpelt; en (4) intenties toestaan dat het systeem de originele richtlijn implementeert terwijl alle lokale wijzigingen op deze richtlijn worden opgeslagen als alternatieve acties.

Ten slotte willen wij het belang van de samenwerking tussen arts en informatiesysteem onderstrepen. De arts kan afwijken van wat de richtlijn voorschrijft of patiëntengegevens toestaan. Het systeem waarschuwt de arts dan en verhindert op deze manier dat de arts een fout maakt, terwijl acties in de geest van de richtlijn wel worden geaccepteerd. Echter, het computersysteem verzamelt data, controleert criteria en voert regels sneller uit dan mensen dat kunnen, maar baseert de adviezen enkel op droge feiten. De arts kan, in tegenstelling tot het systeem, communiceren met de patiënt en de emotionele toestand en behoeften van de patiënt mee laten wegen in de te nemen beslissing. De twee benaderingen gecombineerd resulteren in een zeer krachtig instrument dat de kwaliteit van de zorg aan de patiënt kan verbeteren. De arts behoudt de leiding en blijft eindverantwoordelijk voor de te nemen acties. 
Streszczenie 
Lekarze i pielęgniarki stoją w obliczu szeregu zmian zachodzących w słóżbie zdrowia, takich jak: (1) wprowadzenie elektronicznej dokumentacji pacjenta, (2) implementacja komputerów wspomagających procesy leczenia i zabiegi lekarskie, (3) wzrost jakości leczenia poprzez bazowanie jego na najlepszych dostępnych informacjach na temat skuteczności i efektywności leczenia, oraz (4) rozrost i rozpowszechnianie się wiedzy medycznej, które następuje obecnie w tak szybkim tempie, że większość lekarzy nie jest w stanie nadąrzyć za tym rozwojem.

Stąd rodzi się wielka potrzeba na systemy komputerowe oparte na wiedzy medycznej, które wspierałyby decyzje lekarskie. Zmiany te są interesującym wyzwaniem, aczkolwiek generują również nowe problemy. Jednym z największych wyzwań jest znalezienie kompromisu między dostarczeniem najlepszej terapii (proces) o jak najwyższej jakości (wynik) po jak najniższych kosztach. Protokoły badania klinicznego mogą tutaj pełnić znaczącą rolę. Podczas wizyty lekarskiej, lub podczas obchodu, protokoły badania klinicznego w wersji papierowej nie są łatwe w użyciu. Znalezienie odpowiedniego leczenia jest czasochłonne, a jakość protokołów medycznych nie zawsze jest optymalna. Jedną z dziedzin, która stara się pomóc opiece zdrowotnej znaleźć równowagę między tymi wymaganiami jest specjalizacja nazywana Informatyką Medyczną. Informatyka Medyczna interesuje się wspieraniem opieki zdrowotnej systemami opartymi na technologiach informacyjnych $i$ komunikacyjnych (ICT).

Aby w prawidłowy sposób wspierać działania lekarzy podczas wizyty lekarskiej, muszą takie systemy komputerowe być niezawodne, a ich użytkowanie wkomponowane w rutynę przebiegu leczenia. Poza tym decyzje lekarskie będą jeszcze lepiej wspomagane, kiedy taki system komputerowy ma możliwość wykorzystania danych z elektronicznej dokumentacji pacjenta. W ten sposób komputer może dostarczyć jak najbardziej adekwatną wskazówkę leczenia pacjenta.

W ostatnich latach wiele systemów komputerowych wspomagających decyzje lekarskie (Decision Support System DSS) zostało zrealizowanych, aczkolwiek okazuje się, że takie systemy ograniczają możliwości wyboru leczenia.

Na przykład, jeżeli lekarz nie działa dokładnie według protokołu badania klinicznego, który wprowadzony jest w komputer, ale wykonuje działania które mają tą samą intencję, system wspomagania decyzji lekarskich nadal da ostrzeżenie. W konsekwencji akceptacja takiego systemu przez lekarza może być negatywna, gdyż będzie otrzymywał nadmierne i często niepotrzebne ostrzeżenia.

Jeśli na podstawie badań klinicznych lub przez wprowadzenie nowych leków istnieje możliwość zastosowania nowej, lepszej terapii, system komputerowy wspomagający decyzje lekarskie powinien być skonstuowany w taki sposób, aby nie było większych trudności wprowadzenia nowych informacji do systemu. Ponadto nowy protokół badania klinicznego powinien być wdrożone według odpowiednich opcji. Te opcje są 
określane przez charakterystyki lekarza i pacjenta.

To może wydawać się trochę paradoksalne, że w wielu dziedzinach powstają nowe protokoły badania klinicznego, które mają na calu ograniczenie nadmiernych wariacji w terapiach, a jednocześnie tworzone są systemy komputerowe, kóre mają na celu ułatwienie wprowadzania lokalnych i indywidualnych opcji w leczeniu. Istotne jest, aby przez wprowadzenie protokołów badania klinicznego ograniczyć terapie, które są przestarzałe albo nieefektywne, a przez wprowadzenie komputerowych systemów wspomagających decyzje lekarskie, oferować pomoc na przykład u pacjentów z współwystępującymi chorobami chronicznymi (np: cukrzyca i choroby układu krążenia i nadciśnienie tętnicze). Okazuje się koniecznością tworzenie takich systemów w celu podwyższenia akceptacji komputerowych systemów wspomagających decyzje lekarskie przez samych lekarzy. Podstawowym zadaniem tej pracy doktorskiej jest wprowadzenie intencji i ich zastosowanie w formalizacji protokołów badań klinicznych w wersji elektronicznej. Tekst z wersji papierowej zostaje w formalny sposób przekształcony tak, aby komputer potrafił go zinterpretować Pod termem intencji rozumiemy determinację do działania w określony sposób, to jest abstrakcyjny i nie ilościowo opis celu, który ma być osiągnięty. Na przykład intencją jest „obniżenie ciśnienia krwi”(opis abstrakcyjny), gol jest aby ciśnienie skurczowe było niższe niż $120 \mathrm{mmHg}$, a rozkurczowe niższe niż 80 mmHg (opis ilościowy).

W rozdziale 1 prezentowany jest rozwój i zmiany odnoszące się do stosowania protokołów badań klinicznych w ciągu ostatnich 30 lat. Opisane są definicje różnych pojęć używanych przez komputerowe systemy wspomagające decyzje lekarskie i ich opcje. Przedstawiony jest GASTON, system wymyślony, zaprojektowany i wykonany przy współpracy grupy Informatyki Medycznej na Uniwersytecie w Maastricht i Signal Processing Systems grupy Wydziału Elektrycznego Politechniki w Eindhoven. GASTON jest narzędziem do reprezentowania i formalizowania protokołów badań klinicznych.

Pytaniem, na które w czasie pracy nad tym doktoratem chcieliśmy znaleźć odpowiedzieć jest: Jakie cechy powinien mieć komputerowy systemy wspomagający decyzje lekarskie (albo protokół badania klinicznego), żeby dostarczyć lekarzom więcej swobody w wybieraniu najlepszej terapii dla pacjenta nawet jeśli nie jest ona wedłóg protokołu, ale ma tą samą intencję?. Aby móc odpowiedzieć na to obszerne pytanie, postawionych zostało pięć bardziej konkretnych pytań.

1 Jakie właściwości powinien mieć wysokiej jakości protokół badania klinicznego, i jaka jest najlepsza metoda aby go prezentować w wersji elektronicznej?

2 Jaka powinna być architektura systemu wspomagającego decyzje lakarskie opertego na intencjach?

3 Czy introdukcja intencji rzeczywiście zwiększa swobodę przy podejmowaniu decyzji medycznych? 
4 zy zmiany w protokołach badania klinicznego są łatwiejsze w implementacji przy użyciu intencji?

5 Czy modelowanie protokołów badania klinicznego przy użyciu intencji zmiejsza ich komleksowość?

Rozdział 2 przedstawia wyniki obszernego przeglądu literatury, które zostało przeprowadzone w celu uzyskania informacji na temat czynników wpływających na projektowanie i realizację protokołów badania klinicznego. Ten przegląd literatury koncentruje się na charakterystykach protokołów badania klinicznego, ich rozwój, wdrażania i rozpowszechniania. Omówione zostało jak protokoły badania klinicznego wpływają na proces opieki medycznej i jaki wpływ mają na wyniki leczenia pacjenta. Opisane w literaturze przyczyny nie przestrzegania protokołów badania klinicznego zostały tu przedyskutowane i skomentowane.

Rozdział 3 opisuje rozwój i architekturę systemu do budowy protokoły badania klinicznego w wersji elektronicznej, który nie tylko wspomaga decyzje lekarsje w oparciu o „wyniki najlepszej praktyki”, ale także wspiera alternatywne decyzje. Prezentowany jest zaprojektowany formalizm reprezentacji elektronicznych protokołów przy użyciu intencji i ich realizacja. System nazwany został GASTINE (GASTon INtencyjne Expresje). Ten formalizm został użyty do reprezentacji protokołu leczenia niewydolności serca. Test ten wykazał, że zastosowanie intencji zwieksza swobodę wymaganą w celu uniknięcia niepotrzebnych ostrzeżeń.

Rozdział 4 opisuje, jak protokół leczenia niewydolności serca przy użyciu intencji został zrealizowany w GASTINE. Omawia także deficyty napotkane podczas pierwszej evaluacji systemu i pokazuje, jak te deficyty zostały rozwiązane. W dalszej części rodziału został omówiony sposów w jaki GASTINE rozpoznaje intencje akcji podejmowanych przez lekarza. Następnie zostało przedstawione jak wszystkie kroki protokołu, które doprowadziły do prezentacji obecnego ostrzeżenia są prezentowane użytkownikowi i jak te informacje mogą być użyte w edukacji lekarzy i aktualizaji protokołów.

W rozdziale 5, opisana została weryfikacja systemu GASTINE. Wyniki tej weryfikacji pomagają odpowiedzieć na pytanie, czy system został prawidłowo zaprojektowany $\mathrm{i}$ zaprogramowany.

Przy pomocy prostej elektronicznej dokumentacji pacjenta zaprojektowanej w Microsoft Access został zweryfikowany holenderski protokół niewydolności serca. Ta elektroniczna dokumentacja pacjenta jest połączona z GASTINE. Korzystając z metody Multiple Condition Coverage (MCC) wszystkie możliwe kombinacje wszystkich zmiennych, które zawarte 
są w kondycjach na każdym kroku schematu protokołu reprezentowanego w GASTINE zostały przetestowane. Ponieważ używane przez nas elementy do budowy schematów (nazywane przez nas prymitywami), które wykonują operacje logiczne, były wiele razy przetestowane $w$ innych sytuacja, można było przyjąć, że prymitywy działają poprawnie i ich logika nie musi być testowana. Dlatego też liczba kombinacji badanych wartości może zostać zmniejszona. Dzięki użyciu intencji, schemat protokołu zostaje podzielony na pewną liczbę subdiagramów. W efekcie, liczba kombinacji wartości do badania zostaje jeszcze bardziej ograniczona, co umożliwia nam całkowite przetestowanie systemu i korekcję znalezionych błędów.

Rozdział 6 opisuje walidację GASTINE. Walidacja pomaga odpowiedzieć na pytanie, czy zaprojektowaliśmy odpowiedni system. Czy system spełnia potrzeby użytkownika? Musieliśmy tu udowodnić, że intencje rzeczywiście mają pozytywny wpływ na swobodę działania lekarza. Prowadzą do zmniejszenia liczby niepotrzebnych ostrzeżeń i zapewniają użytkownikowi dostęp do informacji dotyczących przyczyn prezentowanych wskazówek czy ostrzeżeń. Na podstawie danych medycznych od 7 pacjentów (w sumie 70 wizyt lekarskich) została w dwóch fazach przeprowadzona walidacja systemu. W pierwszej fazie wszystkie istotne dane z każdej wizyty lekarskiej (takie jak wyniki laboratoryjne, wyniki badań fizycznych i używane leki) zostały wprowadzone do naszej elektronicznej dokumentacji pacjenta. Na podstawie tych danych GASTINE generowała wskazówki i ostrzeżenia.

Zarówno pro-aktywna jak i re-aktywna metoda działania systemu została tutaj zbadana. Pro-aktywna metoda oznacza, że system generuje wskazówki i ostrzeżenia oparte na danych historycznych zanim lekarz sam podejmie działanie. Re-aktywna metoda oznacza, że system ostrzega użytkownika dopiero kiedy podjęte przez lekarza akcje nie pokrywają się z intencją protokołu medycznego.

W drugiej fazie kardiolog i pielęgniarka wyspecjalizowana w leczeniu niewydolności serca porównali wyniki akcji faktycznie wykonanych podczas leczenia pacjenta z proponowanymi akcjami przez GASTINE, w tym celu mieli też do dyspozycji funkcję cofania, która umożliwia prezentację wszystkich kroków protokołu, które doprowadziły do prezentacji obecnego ostrzeżenia i logikę każdego krogu argumentującą następny krok.

Jedną z konkluzji przeprowadzonej walidacji jest fakt, że system przystępnie reprezentuje swoje rozumowanie jak doszedł do prezentowanej wskazówki czy ostrzeżenia. Zespół, który porównywał akcje podjęte podczas leczenia z tymi generowanymi przez GASTINE doszedł do wniosku, że w 94\% przypadków dawanie ostrzeżenia z dodatkowym wyjaśnieniem było bardzo mile widziane. W praktyce nie przestrzeganie protokołu było dość powszechne. System był w stanie w niemal 50\% przypadków skorygować odstępstwa od protokołu (które nie miały tej samej intencji jak protokół). Wskazane byłoby system testować na większą skalę. 
Rozdział 7 podsumowuje główne wnioski z naszych badania i umieszcza je w kontekście wcześniejszych badań. Ponadto, zostały tu omówione słabe i mocne strony zastosowanego rozwiązania i sugerowane kierunki dalszych badań.

Naszym głównym celem było stworzenie systemu, który dałby lekarzowi więcej swobody podczas leczenia z możliwością działania zgodnie z protokołem, a przynajmniej jego intencji. Dzieki takiemu systemowi, lekarz ma możliwość bazowania leczenia na podstawie zdobytego doświadczenia, lokalnych możliwości (brak aparatury uniemożliwia czasami działanie według protokołu) lub preferencji pacjenta.

Głównym wnioskiem z przeprowadzonych badań opisanych w tej pracy doktorskiej jest fakt, że system wspomagania decyzji lekarskich oparty na intencjach może na różne sposoby pozytywnie wspomagać ochronę zdrowia. Według nas, taki system zmniejsza ryzyko nie przestrzegania protokołów, ponieważ (1) protokoły teraz wspierają pracę lekarza i nie koliduje dając niepotrzebne ostrzeżenia, (2) intencje uproszczaję adaptację protokołów do lokalnych standardów, (3) ewaluacja protokołów wdrożonych przy użyciu intencji jest szybsza i łatwiejsza, i (4) dzięki intencjom protokół wdrożony w systemie jest oryginalnym protokołem, podczas gdy wszystkie lokalne zmiany są zapisywane jako alternatywne działania.

Na zakończenie, chcemy podkreślić znaczenie współpracy między lekarzem i systemem informacji. Leczenie wskazane przez lekarza może się różnić od tego, co protokół nakazuje. System ostrzega lekarza i zapobiega w ten sposób, że lekarz popełni błąd, aczkolwiek działania zgodne z intencją protokołu zostaną zaakceptowane. $Z$ jednej strony, systemem informacji zbiera dane, monitoruje i kontroluje kryteria i sprawdza regóły dużo szybciej niż człowiek byłby w stanie to zrobić, aczkolwiek wskazówka czy ostrzeżenie generowane przez system jest oparta tylko na suchych faktach. Z drugiej strony, lekarz jest w stanie, w przeciwieństwie do systemu komputerowego rozmawiać z pacjentem, ocenić jego stan emocjonalny, wziąć wymagania pacjenta pod uwage przy podejmowaniu decyzji o leczeniu. Dwie strony medalu, które właściwie ze sobą połączone stają się bardzo potężnym narzędziem.Należy jednak podkreślić, że to lekarz ponosi ostateczną odpowiedzialność za działania, które zostaną podjęte. 
Curriculum Vitae 
Agnieszka Anna (Agnes) Latoszek-Berendsen was born in Warsaw, Poland on December $6^{\text {th }}, 1977$.

In 2000 she did three months Erasmus-Socrates foreign scholarship at The Faculty of Physics of the Eindhoven University of Technology in collaboration with the Máxima Medisch Centrum in Veldhoven. She worked on project titled "Wavelet analysis compared to Fourier analysis in blood pressure variability in preterm infants".

In 2002 she obtained her master degree in Biocybernetics and Biomedical Engineering at the Warsaw University of Technology with her work entitled "Design and realization of ultrasound monitor of fetal activity".

In May 2003 she started her PhD study reported in this thesis at the Medical Informatics group of Maastricht University.

In 2008 she started her work for NIPED Prevention Compass on development and implementation of their, powered by GASTON, product.

In 2009 she joined the team of MEDECS BV in the function of Account Manager.

She lives in Heerlen, is married to Ralph and has 2 daughters Eliza and Mijke. 
Publications 
Latoszek-Berendsen, A., Talmon, J., \& Hasman, A. (2006). With good Intentions. Studies in health technology and informatics, 124, 509.

Latoszek-Berendsen, A., Talmon, J., de Clercq, P., \& Hasman, A. (2007). With good intentions. International Journal of Medical Informatics, 76, S440-S446.

Latoszek-Berendsen, A. A., Talmon, J. L., de Clercq, P. A., Gorgels, A. P., \& Hasman, A. (2007). Sensible Decision Support System. In Medinfo 2007: Proceedings of the 12th World Congress on Health (Medical) Informatics; Building Sustainable Health Systems (p. 2206). IOS Press.

Latoszek-Berendsen, A., de Clercq, P., van den Herik, J., \& Hasman, A. (2009). Intentionbased Expressions in GASTINE. Methods of Information in Medicine, 48(4), 391.

Latoszek-Berendsen, A., Tange, H., van den Herik, H. J., \& Hasman, A. (2010). From clinical practice guidelines to computer-interpretable guidelines. Methods Inf Med, 49, 550-570. 
Acknowledgement 
An extraordinary PhD process cannot have an ordinary acknowledgement.

When you start a PhD research everything seems to be straightforward. You will be doing research on a particular topic, with a clear ultimate goal: produce a number of articles and a dissertation showing that you are able to conduct independent research. For reaching this goal you have a whole four years. Especially at the start, this seems to be a very long time... However, those four years do not include all kind of circumstances. In my case, both positive and negative experiences contributed to a process that took slightly longer. The past ten years have learned me, that I would not have reached the original target without the help of many people. Many people who came and went, but left their respective traces behind. Many of you I am grateful for being there, some to assist in contents, some to support me mentally, or just being there for a good talk and laugh. But, most importantly those that were there when I could not go on; there was always one of you who took me by the hand and pulled me up.

After having met so many helpful people in all those years, chances of forgetting a single name at the moment of writing this text are too big so I just do not do it. If you know me then you already know how grateful am I for meeting you on my path and all that you did. Without you, I would not have reached this point.

"When you feel

That you can make it all alone

Remember no one is big enough

To go it all alone"

V. SHEPARD 
SIKS Dissertation Series

189 
2009-01 Rasa Jurgelenaite (RUN)Symmetric Causal Independence Models

2009-02 Willem Robert van Hage (VU)Evaluating Ontology-Alignment Techniques

2009-03 Hans Stol (UVT)A Framework for Evidence-based Policy Making Using IT

2009-04 Josephine Nabukenya (RUN)Improving the Quality of Organisational Policy Making using Collaboration Engineering

2009-05 Sietse Overbeek (RUN)Bridging Supply and Demand for Knowledge Intensive Tasks - Based on Knowledge, Cognition, and Quality

2009-06 Muhammad Subianto (UU) Understanding Classification 2009-07 Ronald Poppe (UT)Discriminative Vision-Based Recovery and Recognition of Human Motion

2009-08 Volker Nannen (VU) Evolutionary Agent-Based Policy Analysis in Dynamic Environments

2009-09 Benjamin Kanagwa (RUN)Design, Discovery and Construction of Serviceoriented Systems

2009-10 Jan Wielemaker (UVA) Logic programming for knowledge-intensive interactive applications

2009-11 Alexander Boer (UVA)Legal Theory, Sources of Law \& the Semantic Web

2009-12 Peter Massuthe (TUE, Humboldt-Universitaet zu Berlin)Operating Guidelines for Services

2009-13 Steven de Jong (UM)Fairness in Multi-Agent Systems

2009-14 Maksym Korotkiy (VU)From ontology-enabled services to service-enabled ontologies (making ontologies work in e-science with ONTO-SOA)

2009-15 Rinke Hoekstra (UVA)Ontology Representation - Design Patterns and Ontologies that Make Sense

2009-16 Fritz Reul (UvT)New Architectures in Computer Chess

2009-17 Laurens van der Maaten (UvT)Feature Extraction from Visual Data

2009-18 Fabian Groffen (CWI)Armada, An Evolving Database System

2009-19 Valentin Robu (CWI)Modeling Preferences, Strategic Reasoning and Collaboration in Agent-Mediated Electronic Markets

2009-20 Bob van der Vecht (UU)Adjustable Autonomy: Controling Influences on Decision Making

2009-21 Stijn Vanderlooy (UM) Ranking and Reliable Classification

2009-22 Pavel Serdyukov (UT)Search For Expertise: Going beyond direct evidence

2009-23 Peter Hofgesang (VU)Modelling Web Usage in a Changing Environment

2009-24 Annerieke Heuvelink (VUA)Cognitive Models for Training Simulations

2009-25 Alex van Ballegooij (CWI)"RAM: Array Database Management through Relational Mapping" 
2009-26 Fernando Koch (UU)An Agent-Based Model for the Development of Intelligent Mobile Services

2009-27 Christian Glahn (OU)Contextual Support of social Engagement and Reflection on the Web

2009-28 Sander Evers (UT)Sensor Data Management with Probabilistic Models

2009-29 Stanislav Pokraev (UT)Model-Driven Semantic Integration of Service-Oriented Applications

2009-30 Marcin Zukowski (CWI)Balancing vectorized query execution with bandwidthoptimized storage

2009-31 Sofiya Katrenko (UVA)A Closer Look at Learning Relations from Text

2009-32 Rik Farenhorst (VU) and Remco de Boer (VU) Architectural Knowledge Management: Supporting Architects and Auditors

2009-33 Khiet Truong (UT)How Does Real Affect Affect Affect Recognition In Speech?

2009-34 Inge van de Weerd (UU)Advancing in Software Product Management: An Incremental Method Engineering Approach

2009-35 Wouter Koelewijn (UL)Privacy en Politiegegevens; Over geautomatiseerde normatieve informatie-uitwisseling

2009-36 Marco Kalz (OUN)Placement Support for Learners in Learning Networks

2009-37 Hendrik Drachsler (OUN)Navigation Support for Learners in Informal Learning Networks

2009-38 Riina Vuorikari (OU)Tags and self-organisation: a metadata ecology for learning resources in a multilingual context

2009-39 Christian Stahl (TUE, Humboldt-Universitaet zu Berlin) Service Substitution - A Behavioral Approach Based on Petri Nets

2009-40 Stephan Raaijmakers (UvT)Multinomial Language Learning: Investigations into the Geometry of Language

2009-41 Igor Berezhnyy (UvT)Digital Analysis of Paintings

2009-42 Toine Bogers (UvT)Recommender Systems for Social Bookmarking

2009-43 Virginia Nunes Leal Franqueira (UT)Finding Multi-step Attacks in Computer Networks using Heuristic Search and Mobile Ambients

2009-44 Roberto Santana Tapia (UT)Assessing Business-IT Alignment in Networked Organizations

2009-45 Jilles Vreeken (UU)Making Pattern Mining Useful

2009-46 Loredana Afanasiev (UvA)Querying XML: Benchmarks and Recursion 
2010-01 Matthijs van Leeuwen (UU) Patterns that Matter

2010-02 Ingo Wassink (UT)Work flows in Life Science

2010-03 Joost Geurts (CWI) A Document Engineering Model and Processing Framework for Multimedia documents

2010-04 Olga Kulyk (UT)Do You Know What I Know? Situational Awareness of Colocated Teams in Multidisplay Environments

2010-05 Claudia Hauff (UT)Predicting the Effectiveness of Queries and Retrieval Systems

2010-06 Sander Bakkes (UvT)Rapid Adaptation of Video Game AI

2010-07 Wim Fikkert (UT)Gesture interaction at a Distance

2010-08 Krzysztof Siewicz (UL)Towards an Improved Regulatory Framework of Free Software. Protecting user freedoms in a world of software communities and eGovernments

2010-09 Hugo Kielman (UL)A Politiele gegevensverwerking en Privacy, Naar een effectieve waarborging

2010-10 Rebecca Ong (UL)Mobile Communication and Protection of Children

2010-11 Adriaan Ter Mors (TUD)The world according to MARP: Multi-Agent Route Planning

2010-12 Susan van den Braak (UU)Sensemaking software for crime analysis

2010-13 Gianluigi Folino (RUN)High Performance Data Mining using Bio-inspired techniques

2010-14 Sander van Splunter (VU)Automated Web Service Reconfiguration

2010-15 Lianne Bodenstaff (UT)Managing Dependency Relations in Inter-Organizational Models

2010-16 Sicco Verwer (TUD)Efficient Identification of Timed Automata, theory and practice

2010-17 Spyros Kotoulas (VU) Scalable Discovery of Networked Resources: Algorithms, Infrastructure, Applications

2010-18 Charlotte Gerritsen (VU)Caught in the Act: Investigating Crime by Agent-Based Simulation

2010-19 Henriette Cramer (UvA) People's Responses to Autonomous and Adaptive Systems

2010-20 Ivo Swartjes (UT)Whose Story Is It Anyway? How Improv Informs Agency and Authorship of Emergent Narrative

2010-21 Harold van Heerde (UT) Privacy-aware data management by means of data degradation 
2010-22 Michiel Hildebrand (CWI) End-user Support for Access tol\ Heterogeneous Linked Data

2010-23 Bas Steunebrink (UU) The Logical Structure of Emotions

2010-24 Dmytro Tykhonov Designing Generic and Efficient Negotiation Strategies

2010-25 Zulfiqar Ali Memon (VU) Modelling Human-Awareness for Ambient Agents: A Human Mindreading Perspective

2010-26 Ying Zhang (CWI)XRPC: Efficient Distributed Query Processing on Heterogeneous XQuery Engines

2010-27 Marten Voulon (UL) Automatisch contracteren

2010-28 Arne Koopman (UU)Characteristic Relational Patterns

2010-29 Stratos Idreos(CWI)Database Cracking: Towards Auto-tuning Database Kernels

2010-30 Marieke van Erp (UvT)Accessing Natural History - Discoveries in data cleaning, structuring, and retrieval

2010-31 Victor de Boer (UVA)Ontology Enrichment from Heterogeneous Sources on the Web

2010-32 Marcel Hiel (UvT)An Adaptive Service Oriented Architecture: Automatically solving Interoperability Problems

2010-33 Robin Aly (UT)Modeling Representation Uncertainty in Concept-Based Multimedia Retrieval

2010-34 Teduh Dirgahayu (UT) Interaction Design in Service Compositions

2010-35 Dolf Trieschnigg (UT)Proof of Concept: Concept-based Biomedical Information Retrieval

2010-36 Jose Janssen (OU) Paving the Way for Lifelong Learning; Facilitating competence development through a learning path specification

2010-37 Niels Lohmann (TUE)Correctness of services and their composition

2010-38 Dirk Fahland (TUE) From Scenarios to components

2010-39 Ghazanfar Farooq Siddiqui (VU)Integrative modeling of emotions in virtual agents

2010-40 Mark van Assem (VU) Converting and Integrating Vocabularies for the Semantic Web

2010-41 Guillaume Chaslot (UM)Monte-Carlo Tree Search

2010-42 Sybren de Kinderen (VU) Needs-driven service bundling in a multi-supplier setting - the computational e3-service approach

2010-43 Peter van Kranenburg (UU) A Computational Approach to Content-Based Retrieval of Folk Song Melodies

2010-44 Pieter Bellekens (TUE)An Approach towards Context-sensitive and Useradapted Access to Heterogeneous Data Sources, Illustrated in the Television Domain 
2010-45 Vasilios Andrikopoulos (UvT) A theory and model for the evolution of software services

2010-46 Vincent Pijpers (VU)e3alignment: Exploring Inter-Organizational Business-ICT Alignment

2010-47 Chen Li (UT) Mining Process Model Variants: Challenges, Techniques, Examples

2010-48 Withdrawn

2010-49 Jahn-Takeshi Saito (UM)Solving difficult game positions

2010-50 Bouke Huurnink (UVA)Search in Audiovisual Broadcast Archives

2010-51 Alia Khairia Amin (CWI)Understanding and supporting information seeking tasks in multiple sources

2010-52 Peter-Paul van Maanen (VU)Adaptive Support for Human-Computer Teams: Exploring the Use of Cognitive Models of Trust and Attention

2010-53 Edgar Meij (UVA)Combining Concepts and Language Models for Information Access

2011

2011-01 Botond Cseke (RUN) Variational Algorithms for Bayesian Inference in Latent Gaussian Models

2011-02 Nick Tinnemeier(UU)Organizing Agent Organizations. Syntax and Operational Semantics of an Organization-Oriented Programming Language

2011-03 Jan Martijn van der Werf (TUE)Compositional Design and Verification of Component-Based Information Systems

2011-04 Hado van Hasselt (UU) Insights in Reinforcement Learning; Formal analysis and empirical evaluation of temporal-difference learning algorithms

2011-05 Base van der Raadt (VU)Enterprise Architecture Coming of Age - Increasing the Performance of an Emerging Discipline.

2011-06 Yiwen Wang (TUE)Semantically-Enhanced Recommendations in Cultural Heritage

2011-07 Yujia Cao (UT)Multimodal Information Presentation for High Load Human Computer Interaction

2011-08 Nieske Vergunst (UU)BDI-based Generation of Robust Task-Oriented Dialogues

2011-09 Tim de Jong (OU)Contextualised Mobile Media for Learning

2011-10 Bart Bogaert (UvT)Cloud Content Contention

2011-11 Dhaval Vyas (UT)Designing for Awareness: An Experience-focused HCI Perspective

2011-12 Carmen Bratosin (TUE)Grid Architecture for Distributed Process Mining

2011-13 Xiaoyu Mao (UvT)Airport under Control. Multiagent Scheduling for Airport Ground Handling

2011-14 Milan Lovric (EUR)Behavioral Finance and Agent-Based Artificial Markets 
2011-15 Marijn Koolen (UvA)The Meaning of Structure: the Value of Link Evidence for Information Retrieval

2011-16 Maarten Schadd (UM)Selective Search in Games of Different Complexity

2011-17 Jiyin He (UVA)Exploring Topic Structure: Coherence, Diversity and Relatedness

2011-18 Mark Ponsen (UM)Strategic Decision-Making in complex games

2011-19 Ellen Rusman (OU)The Mind 's Eye on Personal Profiles

2011-20 Qing Gu (VU)Guiding service-oriented software engineering - A view-based approach

2011-21 Linda Terlouw (TUD)Modularization and Specification of Service-Oriented Systems

2011-23 Wouter Weerkamp (UVA)Finding People and their Utterances in Social Media

2011-24 Herwin van Welbergen (UT)Behavior Generation for Interpersonal Coordination with Virtual Humans On Specifying, Scheduling and Realizing Multimodal Virtual Human Behavior 2011-25 Syed Waqar ul Qounain Jaffry (VU)) Analysis and Validation of Models for Trust Dynamics

2011-26 Matthijs Aart Pontier (VU)Virtual Agents for Human Communication - Emotion Regulation and Involvement-Distance Trade-Offs in Embodied Conversational Agents and Robots

2011-27 Aniel Bhulai (VU)Dynamic website optimization through autonomous management of design patterns

2011-28 Rianne Kaptein(UVA)Effective Focused Retrieval by Exploiting Query Context and Document Structure

2011-29 Faisal Kamiran (TUE)Discrimination-aware Classification

2011-30 Egon van den Broek (UT)Affective Signal Processing (ASP): Unraveling the mystery of emotions

2011-31 Ludo Waltman (EUR)Computational and Game-Theoretic Approaches for Modeling Bounded Rationality

2011-32 Nees-Jan van Eck (EUR)Methodological Advances in Bibliometric Mapping of Science

2011-33 Tom van der Weide (UU)Arguing to Motivate Decisions

2011-34 Paolo Turrini (UU)Strategic Reasoning in Interdependence: Logical and Gametheoretical Investigations 2011-35 Maaike Harbers (UU)Explaining Agent Behavior in Virtual Training

2011-36 Erik van der Spek (UU)Experiments in serious game design: a cognitive approach 2011-37 Adriana Burlutiu (RUN)Machine Learning for Pairwise Data, Applications for Preference Learning and Supervised Network Inference

2011-38 Nyree Lemmens (UM)Bee-inspired Distributed Optimization

2011-39 Joost Westra (UU)Organizing Adaptation using Agents in Serious Games 
2011-40 Viktor Clerc (VU)Architectural Knowledge Management in Global Software Development

2011-41 Luan Ibraimi (UT)Cryptographically Enforced Distributed Data Access Control

2011-42 Michal Sindlar (UU)Explaining Behavior through Mental State Attribution

2011-43 Henk van der Schuur (UU)Process Improvement through Software Operation Knowledge

2011-44 Boris Reuderink (UT)Robust Brain-Computer Interfaces 2011-45 Herman Stehouwer (UvT)Statistical Language Models for Alternative Sequence Selection

2011-46 Beibei Hu (TUD)Towards Contextualized Information Delivery: A Rule-based Architecture for the Domain of Mobile Police Work

2011-47 Azizi Bin Ab Aziz(VU)Exploring Computational Models for Intelligent Support of Persons with Depression

2011-48 Mark Ter Maat (UT)Response Selection and Turn-taking for a Sensitive Artificial Listening Agent

2011-49 Andreea Niculescu (UT)Conversational interfaces for task-oriented spoken dialogues: design aspects influencing interaction quality

\section{2}

2012-01 Terry Kakeeto (UvT) Relationship Marketing for SMEs in Uganda

2012-02 Muhammad Umair(VU)Adaptivity, emotion, and Rationality in Human and Ambient Agent Models

2012-03 Adam Vanya (VU) Supporting Architecture Evolution by Mining Software Repositories

2012-04 Jurriaan Souer (UU)Development of Content Management System-based Web Applications

2012-05 Marijn Plomp (UU) Maturing Interorganisational Information Systems

2012-06 Wolfgang Reinhardt (OU)Awareness Support for Knowledge Workers in Research Networks

2012-07 Rianne van Lambalgen (VU) When the Going Gets Tough: Exploring Agentbased Models of Human Performance under Demanding Conditions

2012-08 Gerben de Vries (UVA)Kernel Methods for Vessel Trajectories

2012-09 Ricardo Neisse (UT) Trust and Privacy Management Support for Context-Aware Service Platforms

2012-10 David Smits (TUE)Towards a Generic Distributed Adaptive Hypermedia Environment

2012-11 J.C.B. Rantham Prabhakara (TUE) Process Mining in the Large: Preprocessing, Discovery, and Diagnostics 
2012-12 Kees van der Sluijs (TUE)Model Driven Design and Data Integration in Semantic Web Information Systems

2012-13 Suleman Shahid (UvT) Fun and Face: Exploring non-verbal expressions of emotion during playful interactions

2012-14 Evgeny Knutov(TUE)Generic Adaptation Framework for Unifying Adaptive Web-based Systems

2012-15 Natalie van der Wal (VU) Social Agents. Agent-Based Modelling of Integrated Internal and Social Dynamics of Cognitive and Affective Processes.

2012-16 Fiemke Both (VU) Helping people by understanding them - Ambient Agents supporting task execution and depression treatment

2012-17 Amal Elgammal (UvT) Towards a Comprehensive Framework for Business Process Compliance

2012-18 Eltjo Poort (VU) Improving Solution Architecting Practices

2012-19 Helen Schonenberg (TUE) What's Next? Operational Support for Business Process Execution

2012-20 Ali Bahramisharif (RUN) Covert Visual Spatial Attention, a Robust Paradigm for Brain-Computer Interfacing 2012-21 Roberto Cornacchia (TUD) Querying Sparse Matrices for Information Retrieval

2012-22 Thijs Vis (UvT)Intelligence, politie en veiligheidsdienst: verenigbare grootheden?

2012-23 Christian Muehl (UT) Toward Affective Brain-Computer Interfaces: Exploring the Neurophysiology of Affect during Human Media Interaction

2012-24 Laurens van der Werff (UT)Evaluation of Noisy Transcripts for Spoken Document Retrieval

2012-25 Silja Eckartz (UT) Managing the Business Case Development in InterOrganizational IT Projects: A Methodology and its Application

2012-26 Emile de Maat (UVA)Making Sense of Legal Text

2012-27 Hayrettin Gurkok (UT)Mind the Sheep! User Experience Evaluation \& BrainComputer Interface Games

2012-28 Nancy Pascall (UvT)Engendering Technology Empowering Women

2012-29 Almer Tigelaar (UT) Peer-to-Peer Information Retrieval

2012-30 Alina Pommeranz (TUD)Designing Human-Centered Systems for Reflective Decision Making

2012-31 Emily Bagarukayo (RUN) A Learning by Construction Approach for Higher Order Cognitive Skills Improvement, Building Capacity and Infrastructure

2012-32 Wietske Visser (TUD) Qualitative multi-criteria preference representation and reasoning

2012-33 Rory Sie (OUN) Coalitions in Cooperation Networks (COCOON)

2012-34 Pavol Jancura (RUN)Evolutionary analysis in PPI networks and applications 
2012-35 Evert Haasdijk (VU) Never Too Old To Learn -- On-line Evolution of Controllers in Swarm- and Modular Robotics

2012-36 Denis Ssebugwawo (RUN) Analysis and Evaluation of Collaborative Modeling Processes

2012-37 Agnes Nakakawa (RUN) A Collaboration Process for Enterprise Architecture Creation

2012-38 Selmar Smit (VU) Parameter Tuning and Scientific Testing in Evolutionary Algorithms

2012-39 Hassan Fatemi (UT) Risk-aware design of value and coordination networks

2012-40 Agus Gunawan (UvT) Information Access for SMEs in Indonesia

2012-41 Sebastian Kelle (OU) Game Design Patterns for Learning

2012-42 Dominique Verpoorten (OU) Reflection Amplifiers in self-regulated Learning

2012-43 Withdrawn

2012-44 Anna Tordai (VU) On Combining Alignment Techniques 2012-45 Benedikt Kratz (UvT) A Model and Language for Business-aware Transactions

2012-46 Simon Carter (UVA) Exploration and Exploitation of Multilingual Data for Statistical Machine Translation

2012-47 Manos Tsagkias (UVA) Mining Social Media: Tracking Content and Predicting Behavior

2012-48 Jorn Bakker (TUE) Handling Abrupt Changes in Evolving Time-series Data

2012-49 Michael Kaisers (UM) Learning against Learning - Evolutionary dynamics of reinforcement learning algorithms in strategic interactions

2012-50 Steven van Kervel (TUD) Ontologogy driven Enterprise Information Systems Engineering

2012-51 Jeroen de Jong (TUD) Heuristics in Dynamic Sceduling; a practical framework with a case study in elevator dispatching

2013

2013-01 Viorel Milea (EUR) News Analytics for Financial Decision Support

2013-02 Erietta Liarou (CWI)MonetDB/DataCell: Leveraging the Column-store Database Technology for Efficient and Scalable Stream Processing

2013-03 Szymon Klarman (VU) Reasoning with Contexts in Description Logics

2013-04 Chetan Yadati(TUD)Coordinating autonomous planning and scheduling

2013-05 Dulce Pumareja (UT) Groupware Requirements Evolutions Patterns

2013-06 Romulo Goncalves(CWI)The Data Cyclotron: Juggling Data and Queries for a Data Warehouse Audience

2013-07 Giel van Lankveld (UT) Quantifying Individual Player Differences 
2013-08 Robbert-Jan Merk(VU)Making enemies: cognitive modeling for opponent agents in fighter pilot simulators

2013-09 Fabio Gori (RUN) Metagenomic Data Analysis: Computational Methods and Applications

2013-10 Jeewanie Jayasinghe Arachchige(UvT)A Unified Modeling Framework for Service Design.

2013-11 Evangelos Pournaras (TUD) Multi-level Reconfigurable Self-organization in Overlay Services

2013-12 Maryam Razavian (VU) Knowledge-driven Migration to Services

2013-13 Mohammad Zafiri (UT) Service Tailoring: User-centric creation of integrated IT-based homecare services to support independent living of elderly

2013-14 Jafar Tanha (UVA) Ensemble Approaches to Semi-Supervised Learning Learning 2013-15 Daniel Hennes (UM) Multiagent Learning - Dynamic Games and Applications

2013-16 Eric Kok (UU) Exploring the practical benefits of argumentation in multi-agent deliberation

2013-17 Koen Kok (VU) The PowerMatcher: Smart Coordination for the Smart Electricity Grid

2013-18 Jeroen Janssens (UvT) Outlier Selection and One-Class Classification

2013-19 Renze Steenhuisen (TUD) Coordinated Multi-Agent Planning and Scheduling

2013-20 Katja Hofmann (UVA) Fast and Reliable Online Learning to Rank for Information Retrieval

2013-21 Sander Wubben (UvT) Text-to-text generation by monolingual machine translation

2013-22 Tom Claassen (RUN) Causal Discovery and Logic

2013-23 Patrício de Alencar Silva (UvT) Value Activity Monitoring

2013-24 Haitham Bou Ammar (UM) Automated Transfer in Reinforcement Learning 

